\title{
Simulation of Water Flow and Retention in Earthen Cover Materials Overlying Uranium Mill Tailings
}
C. S. Simmons
G. W. Gee

September 1981

Prepared for the U.S. Department of Energy under Contract DE-AC06-76RLO 1830

Pacific Northwest Laboratory

Operated for the U.S. Department of Energy by Battelle Memorial Institute 


\title{
NOTICE
}

This report was prepared as an account of work sponsored by the United States Government. Neither the United States nor the Department of Energy, nor any of their employees, nor any of their contractors, subcontractors, or their employees, makes any warranty, express or implied, or assumes any legal liability or responsibility for the accuracy completeness or usefulness of any information, apparatus, product or process disclosed, or represents that its use would not infringe privately owned rights.

The views, opinions and conclusions contained in this report are those of the contractor and do not necessarily represent those of the United States Government or the United States Department of Energy.

PACIFIC NORTHWEST LABORATORY

operated by

BATTELLE

for the

UNITED STATES DEPARTMENT OF ENERGY

Under Contract DE-AC06-76RLO 1830

\author{
Printed in the United States of America \\ Available from \\ National Technical Information Service \\ United States Department of Commerce \\ 5285 Port Royal Road \\ Springfield, Virginia 22151
}

Price: Printed Copy $\$$

$\because$ Microfiche $\$ 3.00$

NTIS

•Pages Selling Price

$\begin{array}{ll}001-025 & \$ 4.00 \\ 026-050 & \$ 4.50 \\ 051-075 & \$ 5.25 \\ 076-100 & \$ 6.00 \\ 101-125 & \$ 6.50 \\ 126-150 & \$ 7.25 \\ 151-175 & \$ 8.00 \\ 176-200 & \$ 9.00 \\ 201-225 & \$ 9.25 \\ 226-250 & \$ 9.50 \\ 251-275 & \$ 10.75 \\ 276-300 & \$ 11.00\end{array}$


UNT / 0203

PNL-3877

33679000597908

UC -70

SIMULATION OF WATER FLOW AND RETENTION IN EARTHEN COVER MATERIALS OVERLYING URANIUM MILL TAILINGS

C. S. Simmons

G. W. Gee

September 1981

Prepared for the U.S. Department of Energy under Contract DE-AC06-76RLO 1830

Pacific Northwest Laboratory Richiand, Washington 99352 


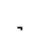


SUMMARY

The water retention characteristics of a multilayer earthen cover for uranium mill tailings were simulated under arid weather conditions common to Grand Junction, Colorado. The multilayer system described in this report consists of a layer of wet clay/gravel (radon barrier), which is separated from a surface covering of fill soil by a washed rock material used as a capillary barrier. The capiliary barrier is designed to prevent the upward migration of water and salt from the tailings to the soil surface and subsequent loss of water from the wet clay. The flow model, UNSATV, described in this report uses hydraulic properies of the layered materials and historical climatic data for two years (1976 and 1979) to simulate long-term hydrologic response of the multilayer system. Application of this model to simulate the processes of infiltration, evaporation and drainage is described in detail. Simulations over a trial period of one relatively wet and two dry years indicated that the clay-gravel layer remained near saturation, and hence, that the layer was an effective radon barrier. Estimates show that the clay-gravel layer would not dry out (i.e., revert to drying dominated by isothermal vapor-flow conditions) for at least 20 years, provided that the modeled dry-climate period continues. 



\section{CONTENTS}

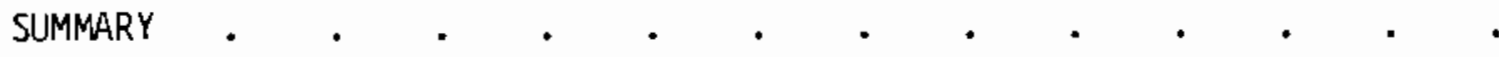

INTRODUCTION

MODELING OF WATER FLOW IN UNSATURATED SOILS

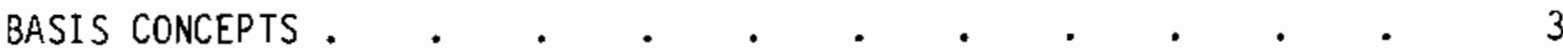

THE MODEL AND INPUT DATA REQUIREMENTS $\quad \cdot \quad \cdot \quad \cdot \quad \cdot \quad \cdot \quad \cdot \quad 4$

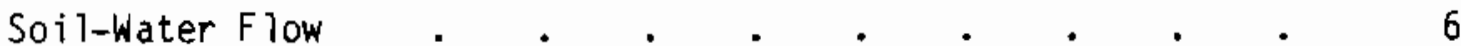

Hydraulic Properties of Soil-Water . . . . . . . 9

Water Retention Characteristics . . . . . . 9

Hydraulic Conductivity . . . . . . . . 10

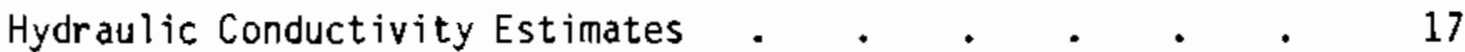

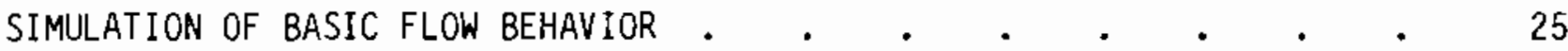

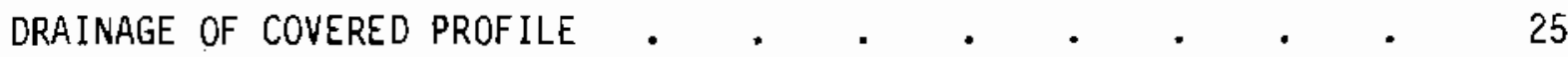

Infiltration . . . . . . . . . . . . 27

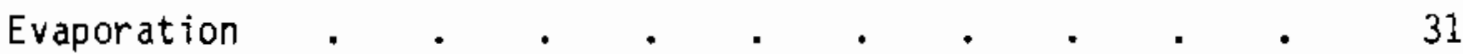

SIMULATION OF THE LAYERED SOIL PROFILE . . . . . . . . . 35

The Climate . . . . . . . . . . . . 36

Potential Evapotranspiration . $\quad . \quad$. $\quad . \quad$. $\quad$. 37

Actual Modeled Evaporation . . . . . . . . . . 42

Plant Roots Sink Model . . . . . . . . . . . . 43

Partitioning Evapotranspiration $\quad . \quad$. $\quad . \quad$. $\quad . \quad 46$

Example Simulation Results . . . . . . . . . . 46

Three-Layer Profile .

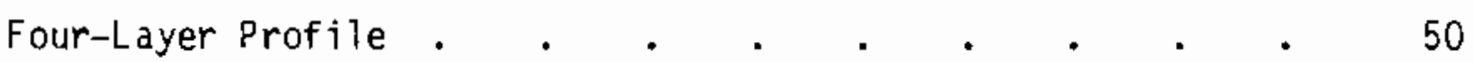

Simulation of a Dry Period . . . . . . . . . . 52 
VAPOR FLOW AND DIFFUSIDN FORMULATION OF SOIL MOISTURE MOVEMENT $\quad$ • $\quad 63$

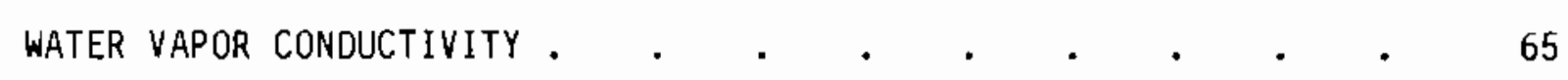

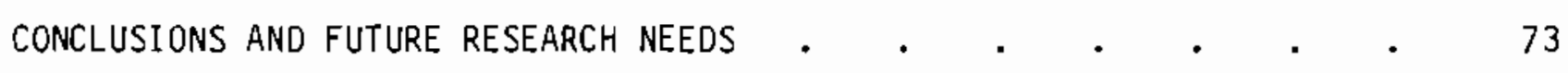
REFERENCES • • • • • • • • • • • • • • • • 75 APPENDIX A - MOISTURE FLOW EQUATION IN FINITE DIFFERENCE FORM • • A.i APPENDIX B - POLYNOMIAL DESCRIPTION DF HYDRAULIC PROPERTIES . . . B. B. APPENDIX C - CAPILLARY CONDUCTIVITY MODELS . . . . . . . . C.1 


\section{FIGURES}

1 Soil-water Characteristics Curves . . . . . . . . . 11

2 Hydraulic Conductivity of Test Materials . . . . . . . . 19

3a Fit of Eq. (11) to the Grand Junction Clay Soij

Characteristic Curve . . . . . . . . . . . 20

3b Hydraulic Conductivity Based on Eq. (12) for Figure 6 . • . 20

4 a Fit of Eq. (11) to the Ciay/Gravel Mix Characteristic Curve . 21

4b Hydraulic Conductivity Based on Eq. (12) for Clay/Gravel

Mix. . . . . . . . . . . . . 21

5 Hydraulic Conductivity Calculated for Medium Tailings by

Six Methods . . . . . . . . . . 22

6 Water Content in a Medium Tailings Profile During Drainage

Without Evaporation. . . . . . . . . . . 28

7 Flux at Various Depths in a Medium Tailings Profile During

Drainage Without Evaporation . . . . . . . . . 29

8 Water Content Profiles at Various Times During Drainage

Without Evaporation. . . . . . . . . . . 30

9 Water Content in a Medium Tailings Profile for a Constant

$1 \mathrm{~cm} /$ day-Infiltration Rate Under Wet Conditions . . . . . 31

10 Water Content in a Medium Tailings Profile for a Constant

$1 \mathrm{~cm} /$ day-Infiltration Rate Under Dry Conditions . . . . 32

11 Flux at Various Depths in a Medium Tajlings Profile for a

Constant $1 \mathrm{~cm} /$ day-Infiltration Rate-Wet Profile Case . . . 33

12 Flux at Various Depths in a Medium Tailings profile for a

Constant $1 \mathrm{~cm} /$ day-Infiltration Rate--Dry Profile Case . . . 34

13 Water Content Profiles During Infiltration Under Wet

Conditions: Hours $1,4,8,16$, and $24 . \quad$. . . . . 35

14 Water Content Profiles During Infiltration Under Wet Con-

ditions: Days 1, 2, 10, 30, and 60 . $\quad . \quad . \quad . \quad . \quad . \quad 36$

15 Water Content Profiles During Infiltration Under Dry

Conditions: Days 1, 2, 10,30, and $60 . \quad$. . . . . . 37 
16 Water Content in a Medium Tailings Profile for Evaporation

Conditions: Depths $2.5,10,50,100 \mathrm{~cm}$. . . . . . 38

17 Flux Near the Surface of a Medium Tailings Profile for

Evaporation Conditions: Depths $0,2.5$, and $10 \mathrm{~cm}$. . . $\quad 39$

18 Flux in the Medium Tailings Profile for Evaporation

Conditions: Depths 50 and $100 \mathrm{~cm}$. . . . . . . 40

19 Water Content Profiles at Various Times During Evaporation

from Medium Tailings: Days 1, 2, 10, 30, and 60 . . . . 41

20 Water Content Profiles Near the Surface for the First Day

of Evaporation from Medium Tailings . . . . . . . 42

21 Water Content in the Three-Layer Profile for the

1979 Climate. . . . . . . . . . . 48

22 Water Content in the Three-Layer Profile with Plants for

the 1979 Climate . . . . . . . . . . . 49

23 Seepage in the Three-Layer Profile, 1979 Climate . . . . 50

24 Seepage in the Three-Layer Profile with Plants,

1979 Climate. . . . . . . . . . . 51

25 Water Storage in the Three-Layer Profile, 1979 Climate,

With and Without Plants. . . . . . . . . 52

26 Water Content Distributions in the Three-Layer Profile,

1979 Climate, With and Without Plants... . . 53

27 Plant Uptake in the Three-Layer Profile, $1979 \mathrm{Cl}$ imate,

Root Zone Depth $80 \mathrm{~cm}$. $. \quad . \quad . \quad . \quad . \quad . \quad .54$

28 Transpiration for Plants in the Three-Layer Profile,

1979 Climate. . . . . . . . . . 55

29 Water Contnt Distributions in the Four Layer Profile with

Plants, 1979 Climate: Days 90, 120, 150, and 350. . . . 56

30 Water Uptake Rate Per Unit or Root Length, $1979 \mathrm{Climate:}$

Depths $2.5,10,30$, and $50 \mathrm{~cm}$. . . . . . . 57

31 Water Content in the Four-Layer Profile with Plant,

First 1976 Climatic Year . . . . . . . . . 58

32 Water Storage to the $80-\mathrm{cm}$ Depth in the Four-Layer Profile,

First and Second 1976 Climatic Year with Plants . . 
33 Water Content Distributions in the Four-layer Profile, First and Second Year with Plants: Days 90, 120, 150, and 350 . . 60

34 Water Vapor Characteristics Curve for Grand Junction Clay . . 61

35 Water Vapor Isotherm for Grand Junction Clay. . . . . . 68

36 Water Vapor Isotherm for Clay/Gravel Mix and Bounding Isotherms Based on $C=1.6$ and $C=7.5$ for Equation (6) $\quad . \quad . \quad . \quad . \quad 69$

37 Soil-water Retention Characteristics of Clay/Gravel Mix

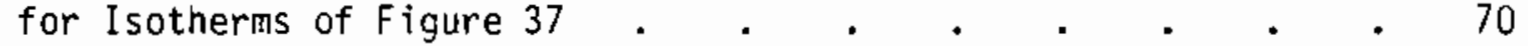

\section{TABLES}

1 Parameters Used to Estimate Hydraulic Conductivity from Capillary Models . . . . . . . . . . . . . . 18

2 Soil-Profile Depth Nodes and Initial Conditions for Simulation of the 1979 Climate of Grand Junction, Colorado . . . . 26

3 Days Required for Plant Roots to Reach Various Depths . . . 45

4 Water Balance in the Three-Layer Profile for 1979 Climate . . 56

5 Water Balance for 1979 Climatic Year Followed by Two 1976 Climatic Years. 



\section{INTRODUCTION}

Prediction of water movement within a soil profile subject to natural climatic conditions is important when studying the possiblity of using mojst soil layers to inhibit radon emissions from uranium mill tailings. The water in a soil directly affects the ease with which radon diffuses in the soi].

Radon, being a gas, moves through porous media such as soil by traveling mainly within air-filled void spaces (Nelson et al. 1980). The gas diffuses from regions of higher to lower gas concentration, provided that the interconnecting pore pathways are not obstructed. Water, which is often present in a soil environment, acts as an obstruction in those pathways. Moreover, because water is denser than radon gas and adheres better to soil, the water will displace the gas. Thus, under the same thermodynamic conditions having equal gas density within pores, soils containing water wiil have a smaller quantity of radon gas per bulk volume than will dry soil. On the other hand, radon's solubility in water complicates describing its movement, and water vapor also competes with radon for the air-filled pore space.

Radon diffusivity is strongly dependent on air-filled porosity and, in a complementary way, on the soil's water content. In wet soil, a small increase in water content may decrease the radon's diffusivity by many orders of magnitude, thereby reducing radon flux in the same proportion. This sensitivity is most likely the result of a relationship between the distribution of pore sizes blocked by water and the soil water content--a behavior similar to water diffusivity in soil but with an opposite water content dependence.

Water movement in a uranium tailings profile as a result of rainfalt, drainage, atmospheric evaporation, and piant transpiration will cause radon concentration and flux to vary considerably, expecialiy near the soil surface where climatic variation is greatest. This report discusses the development of a model called UNSATV, which simulates water flow in unsaturated soils. The model was used to test the feasibility of using a clay-soil layer with high water retention properties as a radon barrier. As indicated by the letter "v" in the model's name, water vapor movement may, in general, have to be considered when assessing a soil layer's long-term ability to retain water. 
A companion report (Mayer et a). 1981) uses the simulated water content profiles generated by the UNSATV model coupled with a radon diffusion model, RND1D, to predict radon diffusion from the multilayer earthen cover system. 
MODELING OF WATER FLOW IN UNSATURATED SOILS

The following section contains a detailed analysis of how water flows through unsaturated soil. The concept of modeling flow in an unsaturated hydrologic zone is explained, and the necessary data input requirements are listed. Examples of infiltration, drainage, and evaporation are presented, and model results for UNSATV are given.

\section{BASIC CONCEPTS}

To understand unsaturated-flow modeling, the physical mechanisms involved require some review. Although this paper simplifies unsaturated-flow modeling into a one-dimensional analysis, the process is actually mechanisticaliy conr plex. The unsaturated zone constitutes the transition region between the atmosphere and a groundwater system. Passage of water through this zone is very dynamic and depends on detailed variations in the hydrautic properties of the water in the soil (henceforth referred to as soil-water). Rain falling directly on the soil surface is usualiy viewed as the primary water input to this zone. But water derived from agricultural irrigation is often a substantial input, too. Seepage beneath rivers and lakes can be described also if the underlying geologicai region is composed essentialiy of granular, soil-type media.

Water moves downward through soil under the influence of gravity as long as there is a sufficient quantity present to overcome the restraining forces of capillary hydraulic potential (matric potential). Water is extracted from the unsaturated zone as surface evaporation and as transpiration by growing plant roots. The rates of both extraction processes depend directly on available solar energy (heat radiation) and surface winds.

Water moves through soil via two physical meachanisms: capillary Darcian flow (liquid phase) and vapor diffusion. Darcian flow is described by hydraulic conductivity and matric potential gradients, both of which manifest extreme variation with even small changes in water content. Vapor diffusion results from thermal gradients and is mainly important near the soil surface, where it controls actual surface evaporation. Maximal potential evaporation rates 
estimated by energy balance methods such as Penman's can be realized only when sufficient water is near the soil surface; otherwise, a dry soil layer limits evaporation.

Water storage by a soil profile is characterized by a water content distribution, which ultimately depends on the detailed spatial variability of hydraulic properties. Infiltrating water that exceeds soil-water holding capacity at a particular profile location will contribute to deep drainage, which may possibly enter the groundwater, depending on depth of the water table. Therefore, a single set of measured hydraulic properties cannot correctiy represent an areal region. An estimate of the statistical distribution is required to determine areal drainage flux. The presence of soil layers further complicates the modeling of unsaturated flow. Soil layers consisting of a variety of distinct media such as clay, silt, sand, and gravel have a physically deterministic effect on water movement patterns. Some layers will rapidly transmit and others will impede soil-water movement. On the other hand, the location and types of various soil layers may be stochastically unknown in a natural soil profile. Such variability contributes substantialiy to the uncertainty in making predictions about unsaturated flow.

Growing plants are powerful and efficient extractors of stored water. The relationship between potential transpiration and climatic conditions must be quantified in an unsaturated-zone simulation. Active plant roots have a water withdrawal distribution that must be taken into account, also. Indeed, an accurate estimation of water balance over an areal region is impossible without detailed quantification and modeling of transpiration ano root growth.

\section{THE MODEL AND INPUT DATA REQUIREMENTS}

The UNSAT computer model (Gupta et al. 1978) was originally developed to describe vertical, one-dimensional, unsaturated water movement under typical agricultural conditions. It was designed as a model for isothermal (i.e., uniform temperature) water flow in the liquid phase. Many models with similar objectives are reported in the literature [e.g., Nimah and Hanks (1973); Hillel (1977); and Feddes et al. (1978)]. A model application by Klute and Heerman (1978) suggested an extension to include isothermal vapor flow. 
General models describing nonisothermal simultaneous flow of both soil-water in two phases and heat have been reported by Hammel (1979) and Sophocleous (1979). All of these unsaturated flow models depend on the quantity and quality of crucial site-specific input data. Indeed, physical phenomena for which no supporting numerical information is available cannot be modeled, regaroless of how complex the model is. In fact, in view of continued revisions of unsaturated flow theory and technology, models should be considered as a research tool aiding understanding and should not be interpreted beyond the limitations of input data. The review by Molz et a1. (1979) provides the current understanding of unsaturated flow.

This report deals specifically with application of the UNSAT model and will discuss an extension to include a partial description of nonisothermal soil-water movement consistent with avajlable input data. In particular, results from the complex nonisothermal models mentioned indicate that heat flow can be decoupled from water flow so that the soil temperature profile can be predicted independently or measured directly and used to estimate associated vapor flux in the water transport. Mathematical details will be discussed in a theoretical section; the variety of input information required for a simulation is reviewed below. The importance of some of the requirements was discussed in a previous application of the model (Gee and Simmons 1979).

The following input information is necessary to define a model simulation of a specific site:

1. Depth of the soil profile and location of each soil layer. The maximum depth is the lower boundary condition location.

2. Type of lower boundary condition specified as a water table or free drainage situation. Free drainage conditions are applicable if the water table is actually deep below the simulated profile.

3. The soil hydraulic properties defined by a soil-water characteristic curve (water retention relationship) and hydraulic conductivity/water content relationship for each soil type present in the profile. 
4. The rainfall and potential evapotranspiration for each day of the simulation period, including the pattern of diurnal variation. Rainfall should be by hour and diurnal variation should be expressed as a fraction of daily amounts for each hour.

5. Properties of the soil-surface vapor-diffusion layer that forms when soil has attained air-dry conditions. These properties include layer thickness and characteristic vapor diffusivity. A water content limit for layer formation is required.

6. The initial water content distribution over the soil profile as established by a water movement history.

7. An optional, measured, soil-temperature distribution if nonisothermal vapor flow conditions are important. A distribution is required for each period with substantial alteration in temperature.

8. Plant growth and water extraction behavior. This information must include a root density distribution as a function of the growth period and actual transpiration as some factor of the potential value when water is not limited.

The above list represents a considerable amount of data. The difficulty of unsaturated flow modeling is that many of the data are seldom available from direct measurements, and so must be estimated by various theoretical methods. Soil-Water Flow

A soil is saturated when all void space (i.e., space not occupied by sojl particles) is filled with water. An unsaturated soil contains air-filled void space as well as water. The measure of water quantity contained by a soil is called water content, which is the volume of water per unit of soil bulk volume. Part of the water in an unsaturated soil will exist as vapor contained in the air-filled pore space, with the amount depending on soil temperature. In view of the greater density of water, relatively moist soils will contain a comparatively smali amount of vapor. Water moves in an unsaturated soil as both liquid and vapor. The movement is always directed from regions of higher to those of lower water potential energy when isothermal conditions prevail. Total water potential energy is expressed as 


$$
\psi=\psi_{p}+\psi_{S}+\psi_{m}+\psi_{z}
$$

where $\psi_{p}, \psi_{s}, \psi_{m}$, and $\psi_{z}$ are the pressure, soiute, matric, and gravitational potentials, respectively. Pressure potential represents external mechanical forces; solute potential represents the attraction forces of water to higher solute concentration (osmotic forces); matric potential represents the adsorptive forces of the soil; and gravitational potential is the energy associated with the water's location in the earth's gravity, measured with respect to some reference point that is usually taken at the soil surface. The negative of the gradient of total potential is the force causing water movement in a soij. In most cases the pressure and solute potential are not present (equal to zero), and the total potential is then called hydraulic potential. When the quantity of water is expressed as weight, the units of potential are centimeters of water. The negative matric potential is also called the pressure head, denoted-h, where $h$ is the positive suction head. In a horizontal soil column, water moves from lower toward higher suction heads. The relationship between pressure head and water content is called the soil-water characteristic curve. This curve is distinct for each soil and is required by the model as input information. A characteristic curve is usually not a single valued functional relationship, because hysteresis effects cause different matric potential values, or energy states, to be associated with a particular water content, depending on whether a soil was dried or wetted to that water content.

Water moves through different soils at different rates for the same hydraufic potential gradient. The hydraulic conductivity, denoted $K(\theta)$, represents the ability of a soil to transmit water at different rates from wet to dry locations. Water flux is described in terms of $K(\theta)$ by Darcy's law:

$$
\underline{q}_{L}=-K(\theta) \frac{\partial \psi}{\partial z}
$$

Hydraulic conductivity, which is a positive function of water content $e$, decreases rapidiy by many orders of magnitude from its maximum saturated value 
as water content decreases. Units of hydraulic conductivity are $\mathrm{cm} / \mathrm{hour}$ when potential and depth $z$ are expressed in cm. Water flux per unit area [Equation (2)] has the same units. Combining Darcy's law--Equation (2)--with the equation for water conservation and expresssing potential in terms of suction head $h$ gives the flow equation

$$
c(h) \frac{\partial h}{\partial t}=\frac{\partial}{\partial z}\left[K(h)\left(\frac{\partial h}{\partial z}+1\right)\right]+S(z, t)
$$

where

$$
c(h)=\frac{-d \theta}{d h}
$$

is the soil-water capacity $\left(\mathrm{cm}^{-1}\right) ; \mathrm{K}(\mathrm{h})$ is expressed explicitly as a function of $h$ by means of the soil-water characteristic, which is usually represented by a single drying curve; and $s(z, t)$ is a piant root sink term in units (cm water/ $\mathrm{cm} \mathrm{so} i l / \mathrm{hr}$ ). Depth $z$ in Equation ( 3 ) is positive downward from the surface, and soil-water flux is given by

$$
q_{L}=K(h)\left(\frac{\partial h}{\partial z}+1\right)
$$

which is positive in the downward direction. The soil-water flow model is described by a finite difference representation of Equation (3) for each soil layer by using an implicit difference solution scheme for $h$, calculated over the profile at each time step. Mathematical details are provided in the appendices. The fundamental asssumption used in soiving Equation (3) is that matric potentiai is a continuous physical quantity in the transition between soil layers. On the other hand, this implies that there is a discontinuous behavior of water content between layers. Accuracy of the finite difference solution is controlled by the mass balance error al lowed over the nodal representation of the soil profile. An interested reader can refer to excellent text books on soil physics (Baver and Gardner 1972; Marshall and Holmes 1979; Hanks and Ashcroft 1980) for further clarification of soil-water flow concepts and measurement of soil-water properties. 
Hydraulic Properties of Soil-Water

The soil-water characteristic curves and hydraulic conductivities constitute those properties required to describe unsaturated water flow. Measurements and calculated estimates of these properties are represented in the UNSAT model by polynomial functions that have been least-squares fit to the data. Polynomial representations used in the computer program avoid large data storage requirements associated with many soil layers and provide a convenient interpolation method. Details of the polynomial descriptions are provided in the appendices. An obvious requirement is that data on soil-water properties must cover the range of water contents expected to be encountered in a particular simulation.

The soil profile modeled consisted of Grand Junction clay, clean rock, a clay/gravei mix, and medium tailings. Data for medium tailings were obtained from Klute and Heermann (1978). Since the time the simulations were run, no data were available for the Grand Junction tailings material. [Since there is a wide variation in material characteristics reported by Klute and Heermann (1978), the medium tailings are as good an estimate of tajlings water retention as required for the simulation.] Data for the other soils were measured for this experiment. The overall objective of the experiment was to study the efficacy of employing a layer of mixed wet clay and gravel to block the escape of radon gas from uranium tailings.

Water Retention Characteristics

Soil-water characteristics were obtained in a manner similar to that used by Klute and Heermann (1978). Pressure head values were measured in the 0 to $-100 \mathrm{~cm}$ range with a suction apparatus with soil cores contacting a porous ceramic plate connected to a hanging water bottle, and in the $-100 \mathrm{~cm}$ to $-5000 \mathrm{~cm}$ range with a pressure chamber apparatus. For pressure head values less than $-5000 \mathrm{~cm}$, the characteristic was determined by a vapor pressure technique in which soil samples are equilibrated in a desiccator over saturated salt solutions that correspond to known relative humidities. The relative humidity, $p / p_{s}$, is related to soil-water potential by: 


$$
\psi=R T \ln \left(p / p_{s}\right)
$$

where

$p=$ water vapor pressure, and $s$ denotes the saturated value

$\mathrm{T}=$ temperature in ${ }^{\circ} \mathrm{K}$

$R=$ gas constant for water vapor, 4.56 bars $/^{\circ} \mathrm{K}$.

The above relationship is based upon an ideal gas law assumption (Baver et al. 1972). Water contents associated with pressure head in each measurement range were determined gravimetrically. A vapor adsorption isotherm relates soil-water content to vapor pressure, and an isotherm is represented by an equation found by Fink and Jackson (1973):

$$
\ln \theta=A+B \ln \left[\left(p / p_{S}\right)^{-C}-1\right]
$$

The parameters of Equation (6) applying to Grand Junction clay soil, for example, are $A=-2.916, B=-0.185$, and $C=1.49$. Retention characteristics shown in Figure 1 are based on the drying curve for each soil.

\section{Hydraulic Conductivity}

Several alternative methods for measuring the hydraulic conductivity of unsaturated soil have been reviewed by Klute (1972). Instantaneous profile methods based on measurements of a draining soil profile can be used to determine conductivity for the relatively wet range (pressure heads greater than $-1000 \mathrm{~cm})$. Philip's infiltration equation provides a method based on an advancing wetting or drying front that can be applied over a considerable range of water contents. Rose (1968) has even used this latter method to determine conductivity within the range of vapor flow. All of these direct measurement methods, however, are experimentally difficult to employ. Moreover, the natural variability of soils makes it difficult to establish a reliable conductivity-water content relation without many duplicate measurements. Because of the complication encountered in measurements of conductivity, a considerable effort has been made to develop theoretical capillary 


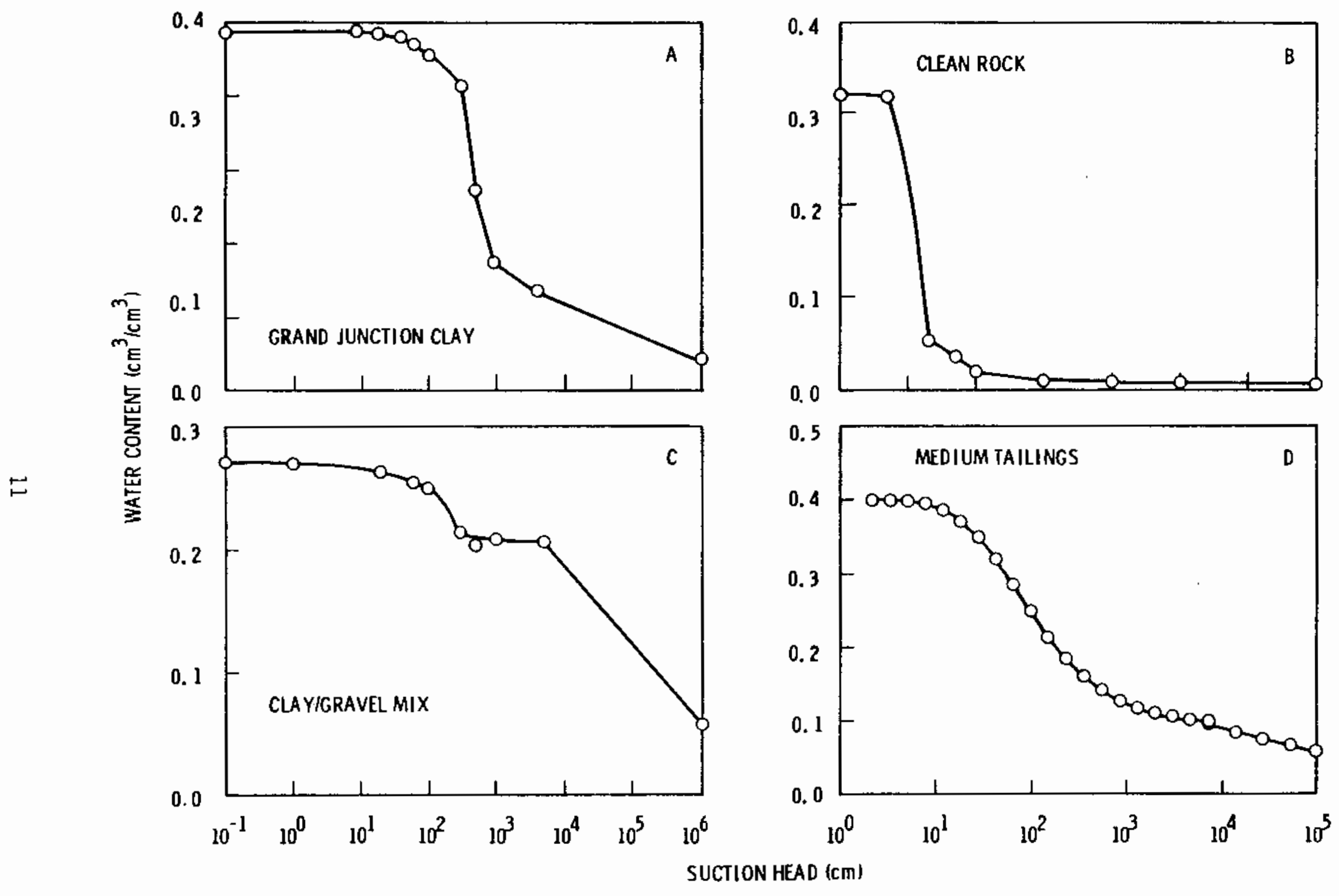

FIGURE 1. Soil-Water Characteristics Curves 
flow models of hydraulic conductivity. Models for predicting hydraulic conductivity of unsaturated soils were reviewed by Mualem (1976), and a new model developed by that author was established for 45 different soils. Mualem's new model, just like all previous similar models, is based on the relationship between the capillary pore size distribution and water retention characteristic. The model equation for relative conductivity $\left[K(\theta) / K_{\text {sat }}\right]$ is

$$
x_{r}(\theta)=s^{n}\left[\int_{0}^{s} d s / \psi / \int_{0}^{1} d s / \psi^{2}\right]
$$

where the effective saturation is

$$
S=\frac{\theta-\theta_{r}}{\theta_{s}-\theta_{r}}
$$

with $\theta_{S}$ and $\theta_{r}$ as the saturated and residual water contents, respectively. The exponent $n$ of Equation (7) was established as $n=1 / 2$ by best $f$ it of the model to measured hydraulic conductivity for the 45 representative soils.

A computer program named HYDRAK was developed to calculate hydrauiic conductivity by Equation (7) and by the similar models based on Burdine's and Millington-Quirk's equations. Those modeis are described by Mualem (1976) and listed in the appendices of this paper. The program evaluates the integrais of Equation (7) and those of the other models by directly integrating along linear or exponential curves connecting the actual measured pressure heads. The exponential interpolation option was included as an extension of the originai linear approach described by Muaiem.

Two important technical difficulties occur in application of capillary models for hydraulic conductivity such as Equation (7). First, the values of relative hydrauic conductivity are extremely dependent on $\theta_{r}$. Residual water content represents the smallest pore size involved in the liquid capillary flow; and when the measured retention characteristic curve does not extend to $\theta_{r}$, the curve must be extrapolated to that water content. Moreover, in many cases the minimum water content is uncertain. Mualem suggested extrapolating 
the characteristic curve and estimating residual water content by fitting the following equation to the end of each measured curve:

$$
\left.\frac{\theta-\theta_{r}}{\theta_{0}-\theta_{r}}=\left(\frac{\psi_{0}}{\psi}\right)^{\lambda} \quad \text { (constant } \lambda\right)
$$

where $\theta_{0}$ and $\psi_{0}$ are the measurements at minimum water content. Using Equation (8) in Equation (7) gives:

$$
k_{r}(\theta)=s^{1 / 2}\left[\varepsilon_{0}+\int_{s 0}^{s} d s / \psi \varepsilon_{0}+\int_{s 0}^{1} d s / \psi\right]^{2}
$$

where

$$
\begin{aligned}
& \varepsilon_{0}=50 /(1+1 / \lambda) \psi_{0} \\
& \text { so }=\left(\theta_{0}-\theta_{r}\right) /\left(\theta_{s}-\theta_{r}\right)
\end{aligned}
$$

There is no theoretical justification of Equation (8), but it provides a standardizing procedure.

The second difficulty involves the behavior of a retention characteristic as pressure head approaches zero for saturated conditions, since then the integrals required in Equations (7) or (9) may not obviously be defined. This is a difficulty that has not been discussed much in the literature. Resolution of the problem appears to require association of a maximum pressure head (minimum suction head) with a physically finite maximum pore size. Some unresolved ambiquity remains, however, because the proper limiting pressure head is uncertain, and the decrease rate in hydraulic conductivity from saturated value depends on that limit. When a capiliary fringe is present, the limit is usually selected as the fringe turning point, which is the minimum pressure head (maximum suction head) such that the characteristic remains level (parallel $\psi$-axis) from saturated water content. However, there does not appear to be justification that the fringe limit or air entry pressure head determines an actual maximum pore size. The answer to this ambiquity might be found in the pore size distribution model of a retention characteristic described by D'Hollander (1979). The model is 


$$
\frac{\theta-\theta_{r}}{\theta_{s}-\theta_{r}}=\int_{0}^{A / \psi} f(r) d r
$$

where

$$
A=-2 \gamma \cos \varepsilon
$$

with $Y$ as the liquid-vapor interface tension, $E$ as the liquid-solid contact angle, and $f(r)$ as the pore size distribution function. Residual water content is the air-dry value $\theta_{r}$. Equation (10) shows that there is an additional degree of freedom in the parameter A associated with liquid-solid interface, and a finite maximum pore radius $r$ can be defined for $\psi$ approaching zero if $A$ also converges to zero with $A / \psi$ remaining finite. Derivations of capiilary hydraulic conductivity models such as Equation (7) assume that $A$ is constant so that it cancels from the formulation, whereas it should perhaps remain to restrict the ranges of integrations in Equation (7).

An analytic solution of Equation (7) was developed by Van Genuchten (1978) that seemingly circumvents the two difficulties in applying Equation (7). A soil-water characteristic was assumed described by the expression:

$$
S=1 / \psi\left[1+(\alpha \psi)^{n}\right] m
$$

with $m=1-1 / n$, where $a$ and $n$ are parameters determined by the $f i t$ to measurements. The relative hydraulic conductivity was shown to be given by:

$$
k_{r}=s^{1 / 2}\left[1-\left(1-s^{1 / m}\right)^{m}\right]^{2}
$$

where the defined relation between parameters $m$ and $n$ was selected to yield the analytic expression Equation (12). It is interesting to note that for large pressure head values, Equation (11) assumes a form similar to the extrapolation Equation (8) used by Mualem. Equation (11) also includes an integrable approximate capillary fringe. The difficulty with Equation (11), 
however, remains that $\theta_{r}$ may not estimate a correct air-dry value, and the hydraulic conductivity may vanish too rapidly in a restricted range of water content. Grand Junction clay soi? and clay/gravel mix provide examples where the discrepancy occurs.

Another simplified analytic formula for hydraulic conductivity was developed by Campbell (1974). Whereas Mualem used a capillary model based on uncorrelated interconnecting pore radii, Campbell's method is based on the other extreme of completely correlated radii. Campbell considered the special case when the retention characteristic is

$$
\psi=\psi\left(\theta / \theta_{5}\right)^{-b}
$$

where $\psi \mathrm{e}$ is the air entry water potential. The derived relative hydraulic conductivity based on Equation (13) is:

$$
x_{r}=(\psi e / \psi)^{2+2 / b}
$$

Implicit in the derivation of Equation (14) is a capillary fringe for the characteristic, i.e., $\theta=\theta_{s}$ when $\psi \geq \psi$ e. Moreover, Equation (13) represents the same form of extension employed by Mualem, Equation ( 8 ), but restricted to the case $\theta_{r}=0$. Another way to obtain Equation (14) is to apply the generalized Burdine equation for capillary conductivity to a special case when the entire characteristic is described by Equation (8) passing through the point $\theta_{S}$, we with $\theta_{r}=0$. Campbell's method is often applicable even when the characteristic is not strictly described by Equation (13). Al1 that is required is that Equation (13) should sufficiently approximate the actual characteristic beyond air entry potential and that Equation (14) be applied only in the water content range where the approximation is applied. Without providing details, it is possible to show that Campbell's method can be made more general by allowing the parameters of Equation (13) to change values at some minimum water content $\theta_{0}$, so that the characteristic can extend with a different slope compatible with measurements. 
In view of the dependency of capiliary models on the extension behavior of water retention characteristics, an improvement in physical justification seems to be possible by using an extended characteristic consistent with the vapor isotherm Equation (6) and water potential Equation (5). This would imply unambiguousiy a minimum residual water content equal to zero for all cases, and the extension of a characteristic would be described asmytotically by:

$$
\theta=\exp \left(A-\frac{8 C}{R T} \psi\right)
$$

Unfortunateiy, Equation (15) does not yield a simple analytic expression for the error term $\varepsilon_{0}$ of the integrals in Equation (9). In many cases, Equation (8) will be adequate when it approximates the water retention characteristic between $\theta_{0}$ and a true air-dry value $\theta_{r}$. Grand Junction clay satisfies that condition, and the absolute error cannot exceed $\theta_{r} /\left|\psi_{r}\right|$.

In order to apply capillary conductivity models, a saturated hydraulic conductivity $k_{\text {sat }}$ must be obtained. Conductivity is then matched to experimental values by taking

$$
K(\theta)=K_{\text {sat }} K_{r}(\theta)
$$

However, any number of other measurements can be used by estimating the best fitting value of $\mathrm{K}_{\text {sat. A final caution on the use of capillary models is }}$ appropriate here. The models are at best simple analogs of the actual physical processes governing water flow. Present models are based on a clearly oversimplified description of pore geometry and its relationship to the soil-water interaction potential. Theoretical estimates of saturated conductivity associated with such models usually do not agree with measurements (Jackson et al. 1965; 8ruce 1972), and it remains a theoretical problem in soil physics to demonstrate why such models describe measurement so we 11 when properly matched to experiment. 
Simplified methods for estimating hydraulic conductivity that use similarity principles for scaling hydraulic properties have been developed also (Bresler, Russo, and Miller 1978). These authors obtained the empirical formula

$$
x(\psi)=k_{\text {sat }}(\psi e / \psi)^{2.6}=k_{\text {sat }} s^{7.2}
$$

based on the scaled regression analysis of a number of soils. K sat was related to the slope with respect to the square root of time of a wetting front distance for entering air-dry soil. Another method based on scaling that would utilize a catalogue of measurements such as Mualem's (1976) has been suggested (Simmons et al. 1979). The method takes advantage of the scale invariance of most capillary conductivity models with respect to a scaled change of $\psi$. That is, if $\psi^{*}$ is a certain reference characteristic having a known relative conductivity, then a scale related $\psi$ with $\alpha \psi=\psi *$ has the same relative conductivity function of water content. A hydraulic conductivity function would be selected with the most similar retention characteristic.

Hydraulic Conductivity Estimates

Hydrauic conductivity for liquid unsaturated flow was estimated from Mualem's Equation (9) for the Grand Junction ciay, clean rock, and clay/gravel mix soils, but that for medium tailings was based on Burdine's equation used by $\mathrm{Xlute}$ and Heermann (1978). The water retention characteristic limits used here for tailings, however, are different than those originally used by $x$ iute. The entire characteristic shown in Figure 1 was used, whereas klute indicates a residual water content 1 imit corresponding to $-7000 \mathrm{~cm}$ pressure head. Table 1 gives the parameter values for each soil, and Figure 2 shows the obtained conductivity dependence on water content. The saturated conductivity for tailings was taken as $60 \mathrm{~cm} /$ day at water content 0.401 from klute's fitted conductivity function

$$
x=2 \times 10^{-7} e^{50 \theta}(\mathrm{cm} / \text { day })
$$


TABLE 1. Parameters Used to Estimate Hydraulic Conductivity from Capillary Modeis

\begin{tabular}{|c|c|c|c|c|}
\hline Soil & ${ }^{\theta} s$ & ${ }^{\theta_{r}}$ & $K_{\text {sat }}(a)$ & $\rho_{b}(a)$ \\
\hline Grand Junction Clay & 0.391 & 0.037 & 0.176 & 1.37 \\
\hline Clean Rock & 0.320 & 0.004 & $3.6 \mathrm{E} 4$ & 1.80 \\
\hline Clay/Gravel Mix & 0.272 & 0.058 & $5.4 E-5$ & 1.84 \\
\hline Medium Tailings & 0.401 & 0.61 & 2.5 & 1.47 \\
\hline
\end{tabular}

(a) $k_{\text {sat }}$ in $\mathrm{cm} / \mathrm{hr}$ and $\rho_{b}$ in $g / \mathrm{cm}^{3}$

The $\theta_{s}$ value was associated with the main drying curve defined by the characteristic curve. For the calculation of conductivity for the clay/gravel mix, an air entry pressure head of $-30 \mathrm{~cm}$ was selected, which introduced an artificial fringe. The importance of introducing a fringe is illustrated in Figure $2 c$ for the clay/gravel mix (Curve II), which shows conductivity based on $\psi e=-0.1 \mathrm{~cm}$ instead. Without a small fringe with at least $\psi \mathrm{e}=-5 \mathrm{~cm}$, the conductivity drops very rapidly from its saturated value we.

Van Genuchten's equations were used also to calculate the conductivity of Grand Junction clay and clay/gravel mix. The least-squares fit of Equation (11) to the characteristic curves is shown in Figures 3 and 4 , and the conductivity by Equation (12) for each soil is also shown. To achieve the fit of Equation (11), the measured air dry point was removed for each soil. It is clear that Equation (11) substantialiy overestimates the residual water content in each case, and that hydraulic conductivity at low water contents deviates greatly from estimates shown in Figure 2. Capiliary conductivity by Equation (12) vanishes below a 0.205 water content for clay/gravel mix, but there remains a considerable contribution to capillary flow.

The graphs in Figure 5 demonstrate the calculation of hydraulic conductivity by six capillary models for medium tailings. This soil provides a good subject for comparison since Klute and Heermann (1978) obtained measurements near minimum water content. The exponential function, Equation (18), used to represent conductivity is shown in Figure $5,(K)$. It is linear on the 


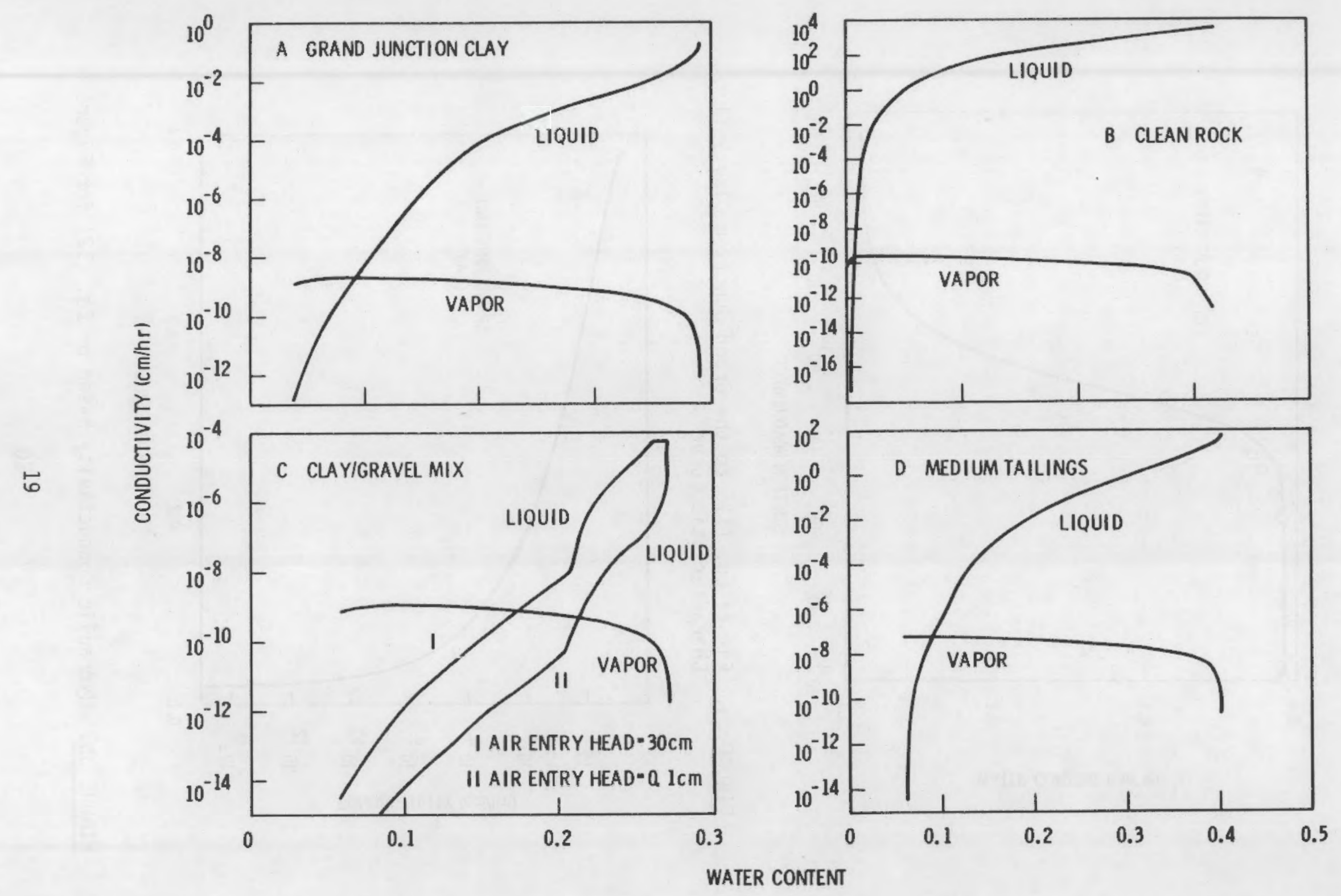

FIGURE 2. Hydraulic Conductivity of Test Materials 


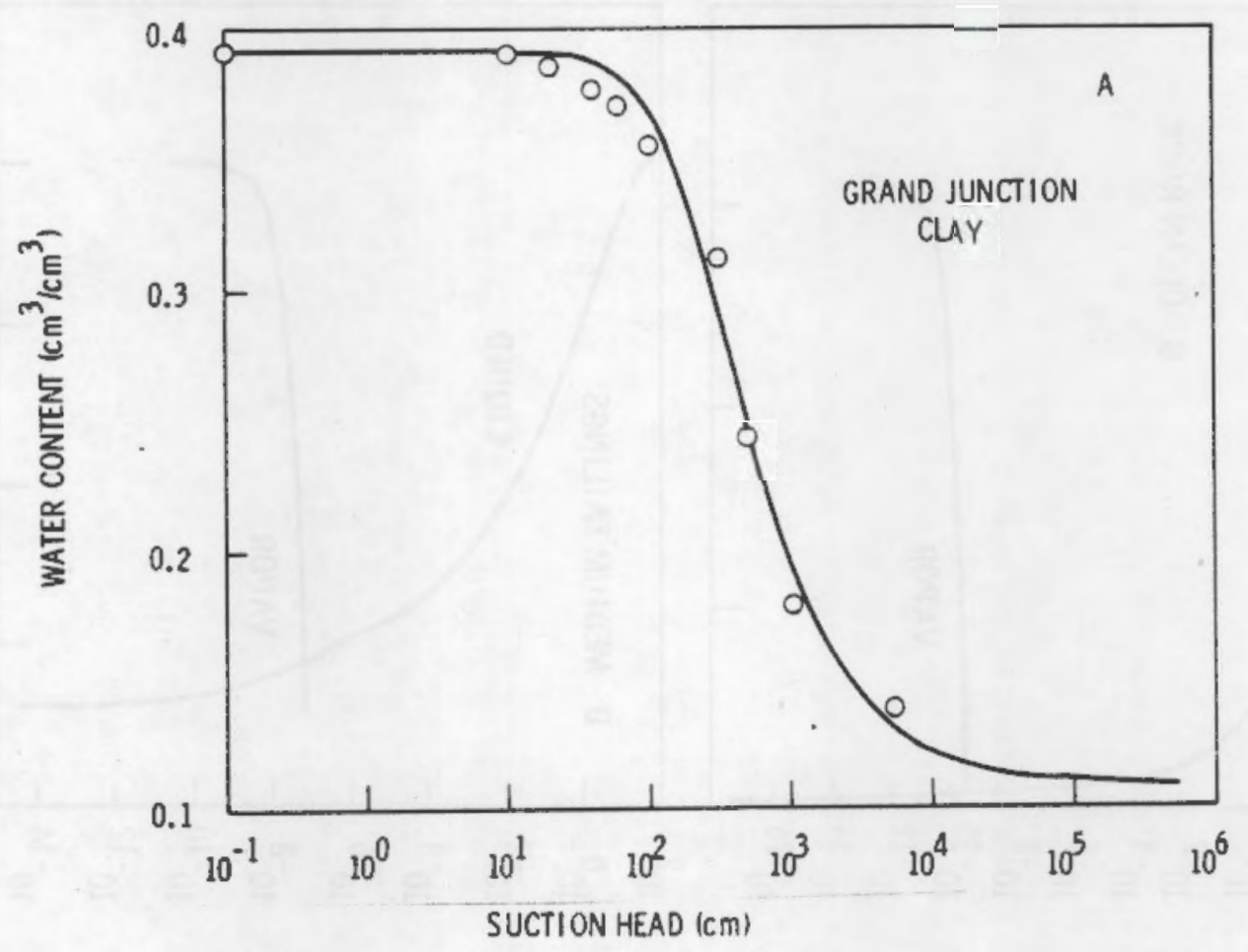

FIGURE 3a. Fit of Eq. (11) to the Grand Junction Clay Soil Characteristic Curve

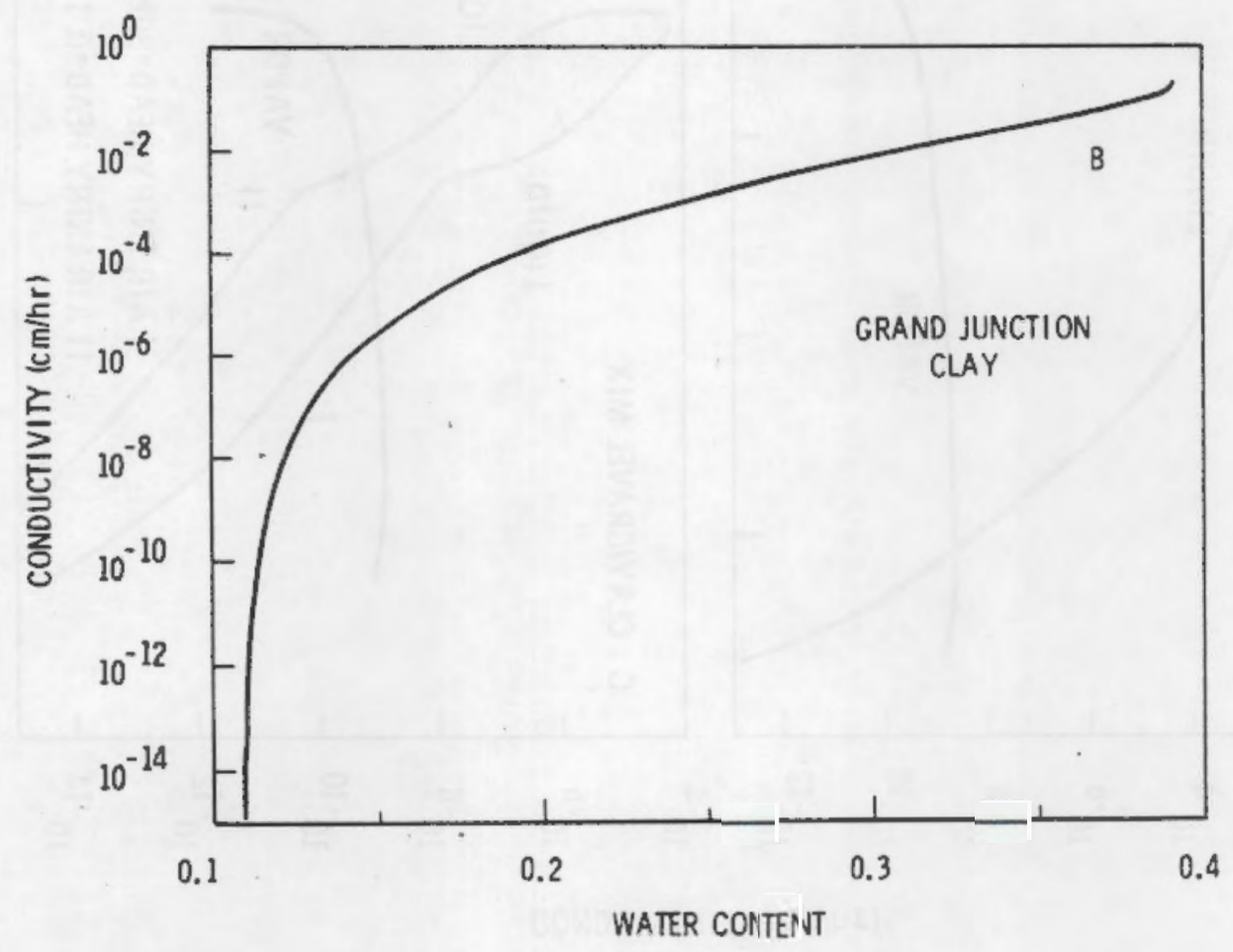

FIGURE 3b. Hydraulic Conductivity Based on Eq. (12) for Figure 6 


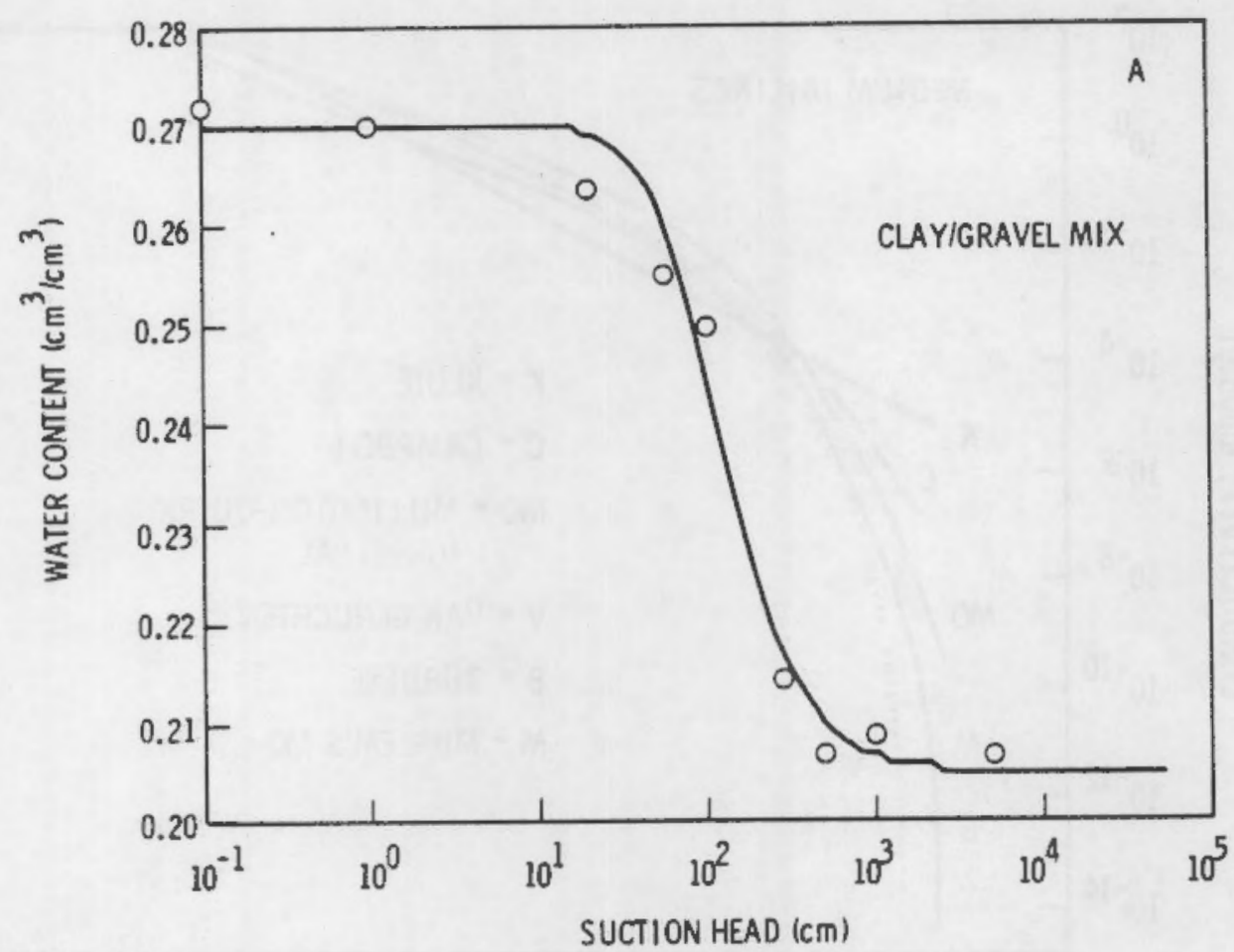

FIGURE 4a. Fit of Eq. (11) to the Clay/Gravel Mix Characteristic Curve

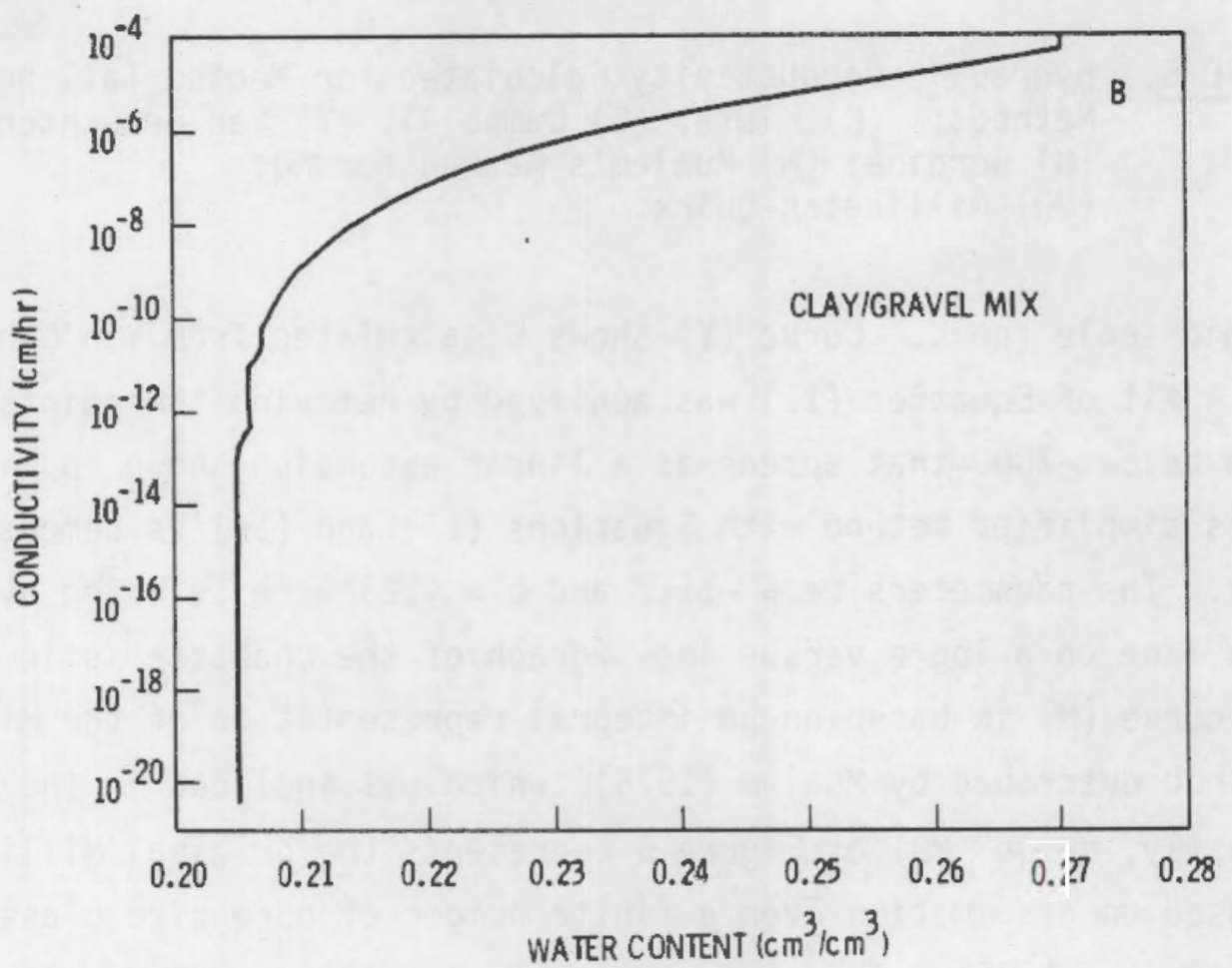

FIGURE 4b. Hydraulic Conductivity Based on Eq. (12) for Clay/Gravel Mix 


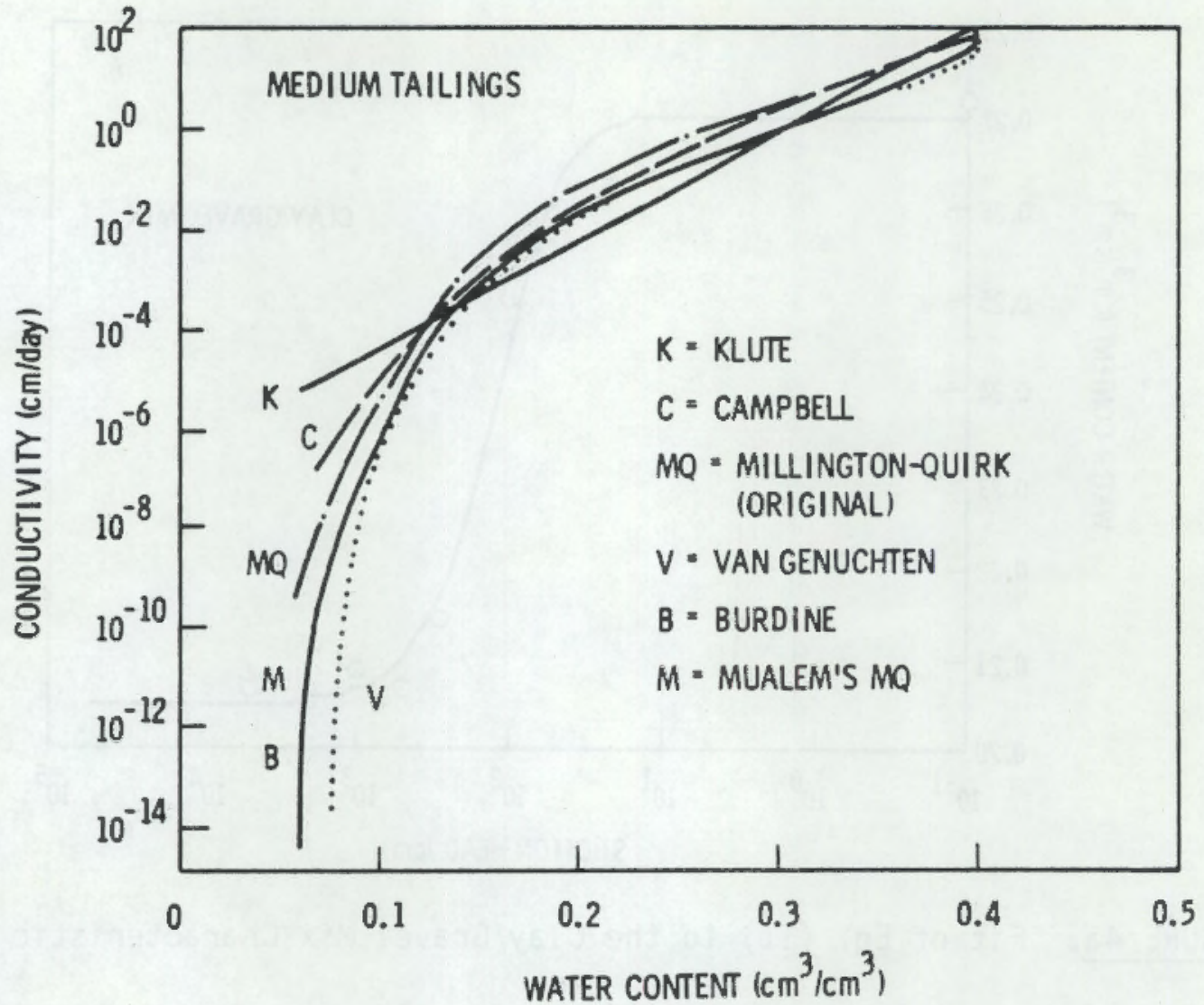

FIGURE 5. Hydraulic Conductivity Calculated for Medium Tailings by Six Methods: (K) Klute; (C) Campbel1; (V) Van Genuchten;

(B) Burdine: (M) Mualem's Method for MQ; (MQ) Millington-Quirk.

logarithmic scale for K. Curve (V) shows K calculated from Van Genuchten's method. A fit of Equation (11) was achieved by removing the points with pressure head below -7000 that appear as a linear extension shown in Figure 1c. Campbell's simplified method with Equations (13) and (14) is demonstrated by curve $(C)$. The parameters $\psi \mathrm{e}=-11.2$ and $\mathrm{b}=4.23$ were selected by superposition of a line on a $\log \theta$ versus $\log -\psi$ graph of the characteristic curve. In Figure 5 curve $(M)$ is based on an integral representation of the MillingtonQuirk method described by Mualem (1976), which was included in the HYDRAK program. Lastly, curve (MQ) of Figure 5 represents the original Millington-Quirk method based on a summation over a finite number of pore size classes (Klute 1972, Reisenauer 1972). The various methods are also compared with the Burdine method in Figure 5, indicated by $(B)$. The new Millington-Quirk method 
(M) essentially coincided with Burdine's method and only one curve is shown. All methods predict conductivity decreasing below $\theta=0.1$ more than the actual measured values represented by $(K)$. Campbell's method (C) appears to remain nearest, most likely because Equation (13) describes the least decrease in pressure head as water content decreases. Such behavior is associated with a greater population of larger pore radii. The result that all capillary model conductivities are below actual measurements seems to indicate that an enhanced vapor flow was present that contributed to conductivity, which was not accounted for. These results for tailings are similar to those obtained by Gee et al. (1981), who suggested that vapor enhancement of the conductivity would explain observed differences between calculated and measured conductivities in the low water content range for a sandy soil material.

To demonstrate the importance of the specific capillary fringe, the Burdine conductivity was calculated again using $\psi$ e equal to $-0.1 \mathrm{~cm}$ instead of the $-2 \mathrm{~cm}$ given by Klute for medium tailings. The associated saturated water content was increased by 0.0001 so that the altered characteristic would not have a fringe between $-2 \mathrm{~cm}$ and $-0.1 \mathrm{~cm}$. The conductivity was then found to decrease to approximately half of the original value at each water content. This result clearly demonstrates the importance of a precisely determined retention characteristic of near saturated conditions. These observations and repetitions of calculations with a wide variety of soil materials and we values suggest that the matching factor used in the Millington-Quirk and similar models is altered if a capillary fringe is not used. We suggest that a capillary fringe must always be used or simulated for the wettest water retention data point. 



\section{SIMULATION OF BASIC FLOW BEHAVIOR}

Unsaturated soil-water flow involves drainage, infiltration, and evaporation as basic flow behavior. Drainage occurs in a soil profile when water under the influence of gravity leaves through the bottom. The profile bottom is usually a saturated groundwater table that receives the drainage. Infiltration is the entry of water through the soil surface, and evaporation is the removal of water. Whenever a system is not static, redistribution will occur. Redistribution is simply the movement of water. Before we proceed with the discussion about simulating water movement in the four-layer soil profile as influenced by natural climatic conditions, we will demonstrate the three basic types of flow behavior. The simulations can be viewed also as a verification of proper functioning of the mathematical model.

Medium tailings material was selected for these comparison simulations, since it is the major component of the soil profile. To facilitate comparison, the same nodalization of the soil profile was used in both the homogeneous and layered profile simulation. The locations of the depth nodes used to describe the 7.63-meter-deep profile are given in Table 2.

\section{DRAINAGE OF COVERED PROFILE}

Drainage of the covered profile without evaporation was simulated for 60 days. Initial conditions in the profile were uniform water content of 0.247 and $-100 \mathrm{~cm}$ pressure head from the surface to the 6.5 meter depth and an equilibrium water content distribution between the water table and the 6.5-meter depth. An equilibrium distribution above the water table was allowed so that drainage flux would not include an appreciable infiltration from the water table if a $-100 \mathrm{~cm}$ pressure head had been applied to the entire profile. The initial conditions correspond to unit hydraulic gradient conditions above 6.5-meter depth and $c$ an be viewed as having been established by a constant surface flux. Steady ponding of water on the surface is an experimental technique that has been used to study subsequent drainage in a field soil (Nielsen, Biggar, and Ehr 1973). The methods of those authors can be used to obtain approximate analytical descriptions of drainage that are useful for comparison purposes. 
TABLE 2. Soil-Profile Depth Nodes and Initial Conditions for Simulation of the $1979 \mathrm{Cl}$ imate of Grand Junction, Colorado

\begin{tabular}{|c|c|c|c|}
\hline Soil Layer & Depth, cm & $\begin{array}{l}\text { Suction } \\
\text { Head, cm }\end{array}$ & $\begin{array}{c}\text { Water } \\
\text { Content }\end{array}$ \\
\hline $\begin{array}{l}\text { Grand Junction } \\
\text { Clay }\end{array}$ & $\begin{array}{r}0.0 \\
2.5 \\
5.0 \\
7.5 \\
10.0 \\
15.0 \\
20.0 \\
25.0 \\
30.0 \\
35.0 \\
40.0 \\
50.0 \\
60.0 \\
70.0 \\
80.0 \\
99.0\end{array}$ & $\begin{array}{r}1450.0 \\
1367.0 \\
1293.0 \\
1230.0 \\
1176.0 \\
1090.0 \\
1025.0 \\
975.0 \\
935.0 \\
902.0 \\
874.0 \\
830.0 \\
796.0 \\
768.0 \\
746.0 \\
709.0\end{array}$ & $\begin{array}{l}0.170 \\
0.172 \\
0.175 \\
0.177 \\
0.179 \\
0.183 \\
0.187 \\
0.190 \\
0.192 \\
0.195 \\
0.197 \\
0.200 \\
0.203 \\
0.206 \\
0.208 \\
0.212\end{array}$ \\
\hline Clean Rock & $\begin{array}{l}100.0 \\
110.0 \\
120.0 \\
129.0\end{array}$ & $\begin{array}{l}709.0 \\
709.0 \\
709.0 \\
709.0\end{array}$ & $\begin{array}{l}0.008 \\
0.008 \\
0.008 \\
0.008\end{array}$ \\
\hline $\begin{array}{l}\text { Clay/Gravel } \\
\text { Mix }\end{array}$ & $\begin{array}{l}130.0 \\
132.0 \\
135.0 \\
140.0 \\
142.0 \\
144.0\end{array}$ & $\begin{array}{l}5.0 \\
5.0 \\
5.0 \\
5.0 \\
5.0 \\
5.0\end{array}$ & $\begin{array}{l}0.266 \\
0.266 \\
0.266 \\
0.266 \\
0.266 \\
0.266\end{array}$ \\
\hline $\begin{array}{l}\text { Medium } \\
\text { Tailings }\end{array}$ & $\begin{array}{l}145.0 \\
150.0 \\
160.0 \\
180.0 \\
200.0 \\
250.0 \\
300.0 \\
400.0 \\
500.0 \\
600.0 \\
650.0 \\
700.0 \\
750.0 \\
755.0 \\
760.0 \\
763.0\end{array}$ & $\begin{array}{r}651.0 \\
646.0 \\
629.0 \\
612.0 \\
590.0 \\
524.0 \\
467.0 \\
365.0 \\
265.0 \\
165.0 \\
115.0 \\
65.0 \\
15.0 \\
10.0 \\
5.0 \\
2.0\end{array}$ & $\begin{array}{l}0.141 \\
0.141 \\
0.142 \\
0.144 \\
0.145 \\
0.150 \\
0.155 \\
0.167 \\
0.185 \\
0.214 \\
0.238 \\
0.278 \\
0.370 \\
0.384 \\
0.399 \\
0.408\end{array}$ \\
\hline
\end{tabular}


Figure 6 shows the water content as a function of time at some selected depths. If drainage continued for an indefinite time, the profile would attain a static state with zero flux throughout and a suction head equal to the distance above the water table plus the value of suction head fixed at the water table $(2 \mathrm{~cm}$ in this case). Figure 7 shows flux at some of the selected depths. Downward flux is positive. Flux near the surface nearly vanishes in 60 days, but flux deep in the profile remains nearly constant as water is supplied by drainage from above. Figure 8 displays the water content profiles at various times.

Storage is the total amount of water held in the profile at any time. The initial conditions of the drainage case constituted $195.4 \mathrm{~cm}$ of storage, while the storage at the end of 60 days was $188.2 \mathrm{~cm}$. A model prediction of $7.22 \mathrm{~cm}$ of cumulative drainage into the water table resulted. For purposes of comparison with the other cases, we note that seepage (cumulative flux integrated over time) at the $100-\mathrm{cm}$ depth was $3.28 \mathrm{~cm}$.

\section{Infiltration}

Infiltration occurs when rain falls on the surface of the soil. The water from an intense rainstorm may be entirely absorbed by a permeable soil such as sand, but a relatively impermeable clay soil might reject most of the applied water as surface runoff. The UNSAT model can simulate such infiltration behavior. As an example, a constant flux of $1 \mathrm{~cm} /$ day was applied to the surface of the medium tailings profile. Because medium tailings have a $60-\mathrm{cm} / \mathrm{day}$ saturated conductivity, the infiltration rate equaled the rainfall rate, and all applied water was taken into the soil. In an actual field situation, however, the impact of raindrops can decompose soil particles and hydraulic conductivity can be reduced by the filling of soil pores with mobile, fine soil material. The soil surface can also become blocked with air if air is unable to escape as water enters the soil. No attempt has been made in the UNSAT model to describe these complicated processes, and so the water input rate is controlled by a given fixed conductivity function.

The infiltration test simulations were performed with two sets of initial conditions corresponding to wet and dry profile conditions. The same initial 


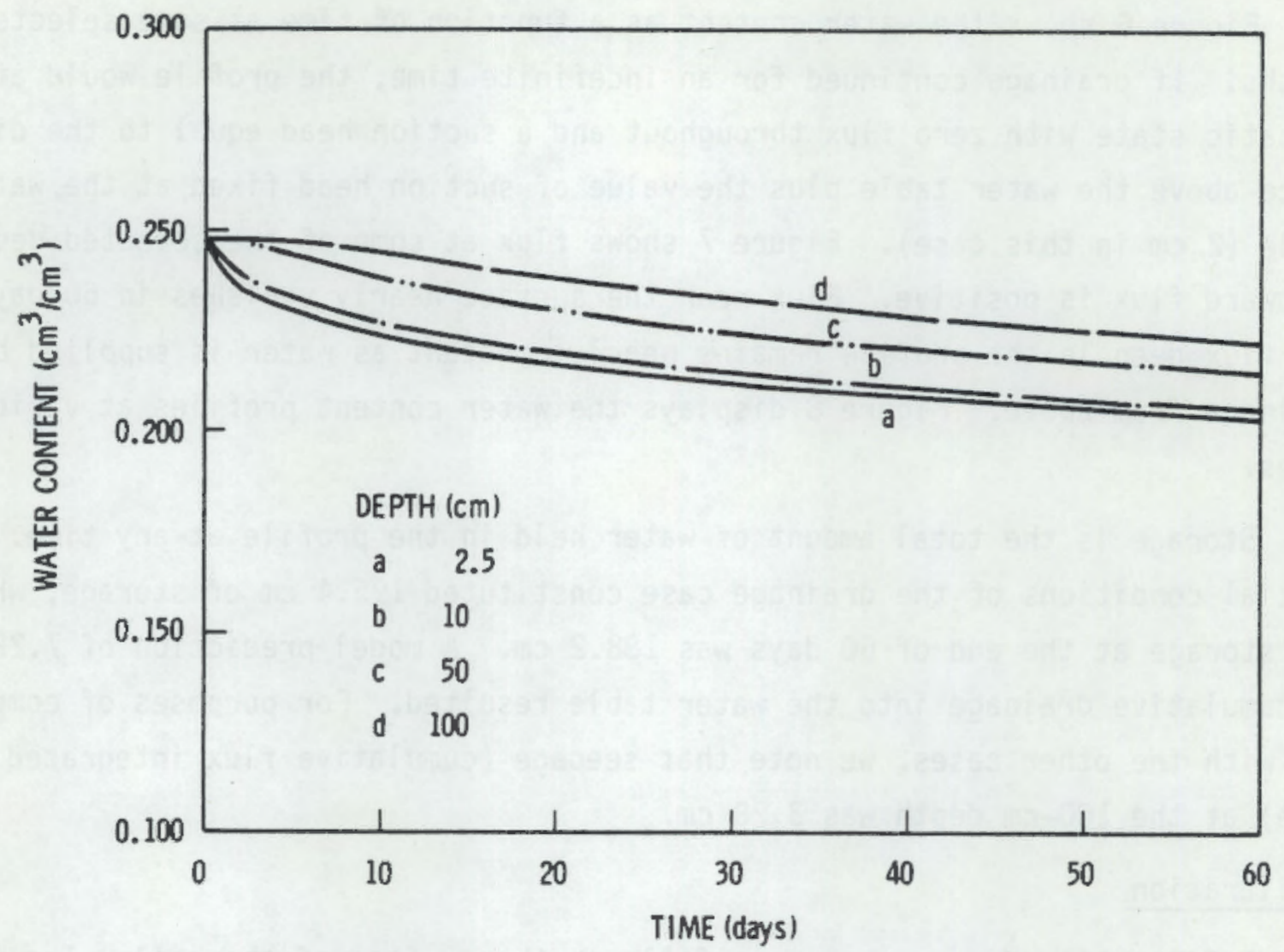

FIGURE 6. Water Content in a Medium Tailings Profile During Drainage Without Evaporation: Depths 2.5, 10, 50, and $100 \mathrm{~cm}$

conditions for the drainage simulation defined the wet profile and, for the dry profile, a $-10^{4} \mathrm{~cm}$ pressure head replaced the $-100 \mathrm{~cm}$ value. In the wet profile the previous drainage was simultaneously simulated with infiltration, whereas little drainage obscured infiltration into the dry profile. In general, infiltration and movement of the associated wetting front is the most difficult problem of unsaturated flow simulation. In order to maintain accuracy, the time-step size and node spacing must be sufficiently smail to resolve the precipitous change in water content and hydraulic properties encountered for a wetting front. On the other hand, requiring too small a time step can make the simulation inefficient and impractical. The UNSAT model uses a timestep control based on the accuracy of the water mass balance, which heips to reduce infiltration simulation problems. Unequal node spacing in the model 


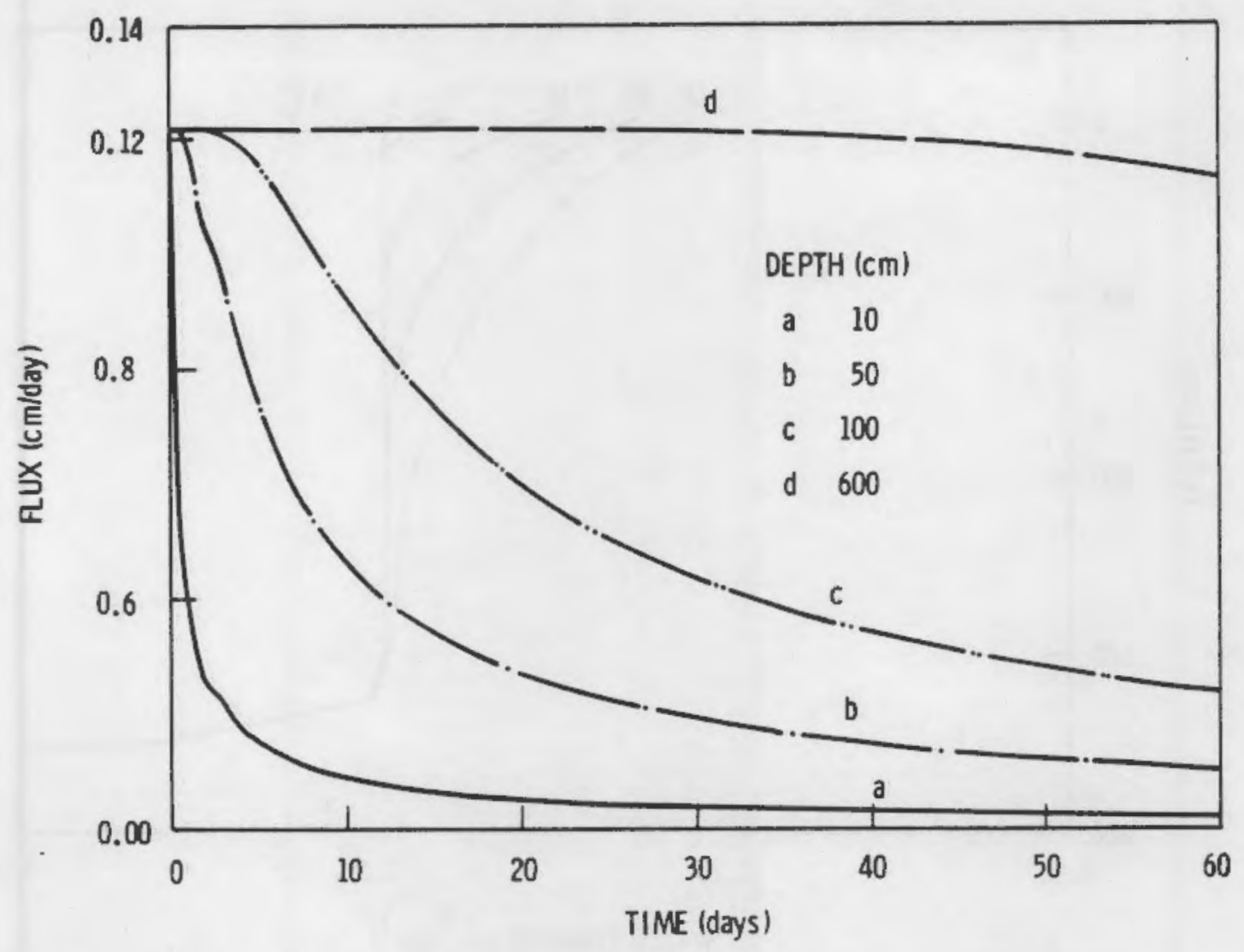

FIGURE 7. Flux at Various Depths in a Medium Tailings Profile During Orainage Without Evaporation

allows depth nodes to be concentrated near the surface, where the most rapid water movement takes place. Approximate analytical solutions derived by Philip (1957) and white et al. (1979) can serve as valuable checks if the simulation accuracy is uncertain, but such a comparison is not attempted here.

Figures 9 and 10 show the time dependence of the water content for the wet and dry initial conditions, respectively. Figures 11 and 12 display corresponding flux time dependence. Water content is seen to increase regularly from its initial to steady final value at all depths, except for $600 \mathrm{~cm}$. Flux increases from an initial drainage value to the final steady value equal to applied surface flux. A flux overshoot, however, is apparent for the $600-\mathrm{cm}$ depth. Water content profiles at various times are shown in Figures 13 through 15. Figure 13 shows a regular advance of water near the surface, which is 


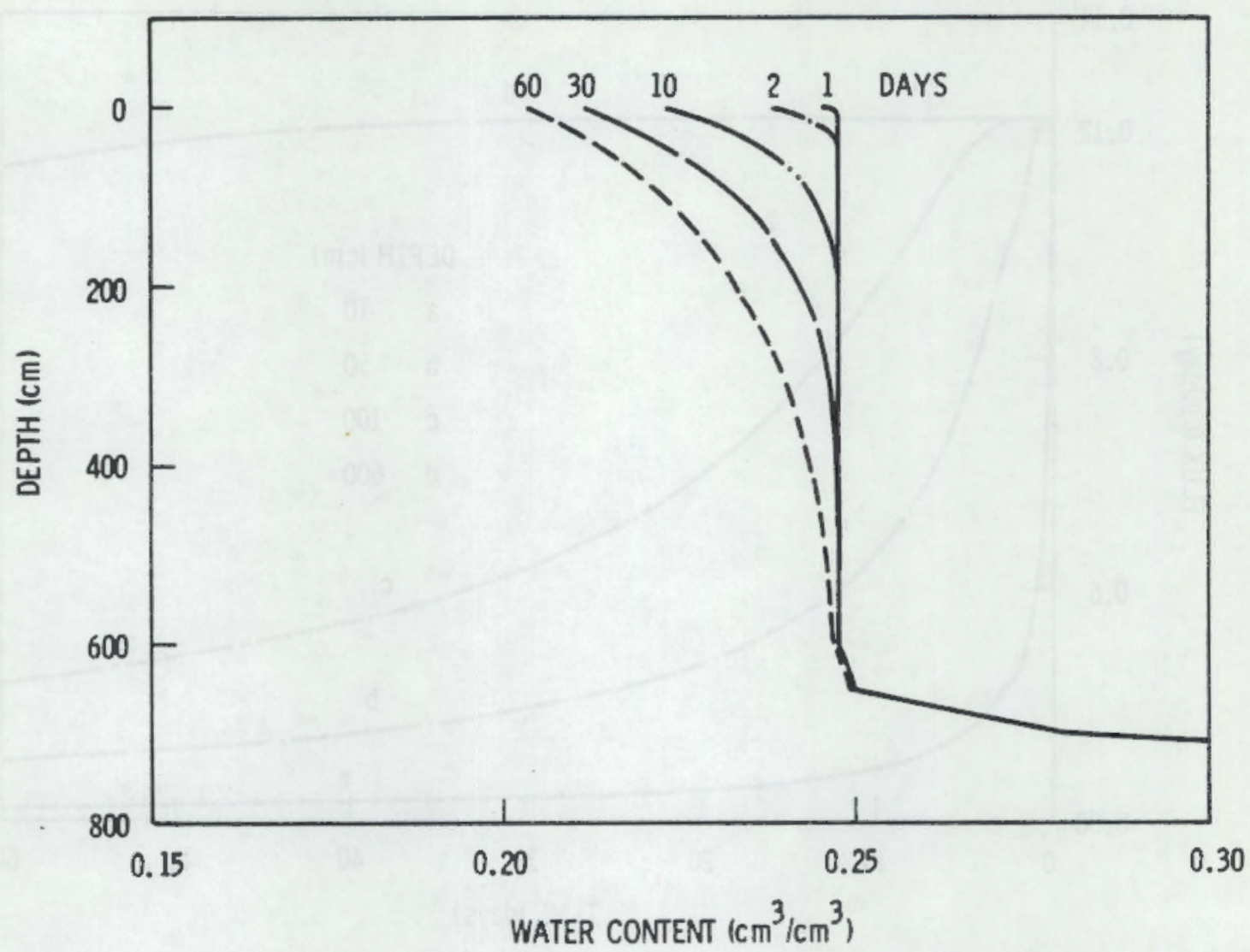

FIGURE 8. Water Content Profiles at Various Times During Drainage Without Evaporation: Days 1, 2, 10, 30, and 60

representative of rainfall. Figure 14 for wet initial conditions seems to indicate numerical oscillation errors as the wetting front approaches the water table and is passing below $300 \mathrm{~cm}$, where the node spacing becomes coarse. In the simulation of actual climatic conditions, the natural rainfall events would not penetrate as deeply. Figure 15 shows the advance of the wetting front into a dry profile, and water is seen to move up from the water table.

Storage in the wet profile increased to $226.8 \mathrm{~cm}$ after $60 \mathrm{~cm}$ of infiltration. Drainage was $28.63 \mathrm{~cm}$, indicating seepage of $21.4 \mathrm{~cm}$ of water more than given by the drainage case. Seepage at the $100-\mathrm{cm}$ depth was $55.46 \mathrm{~cm}$, indicating that $52.2 \mathrm{~cm}$ of infiltrated water passed this depth. The dry profile showed a change in storage from an initial $96.2 \mathrm{~cm}$ to a final $163.1 \mathrm{~cm}$. 


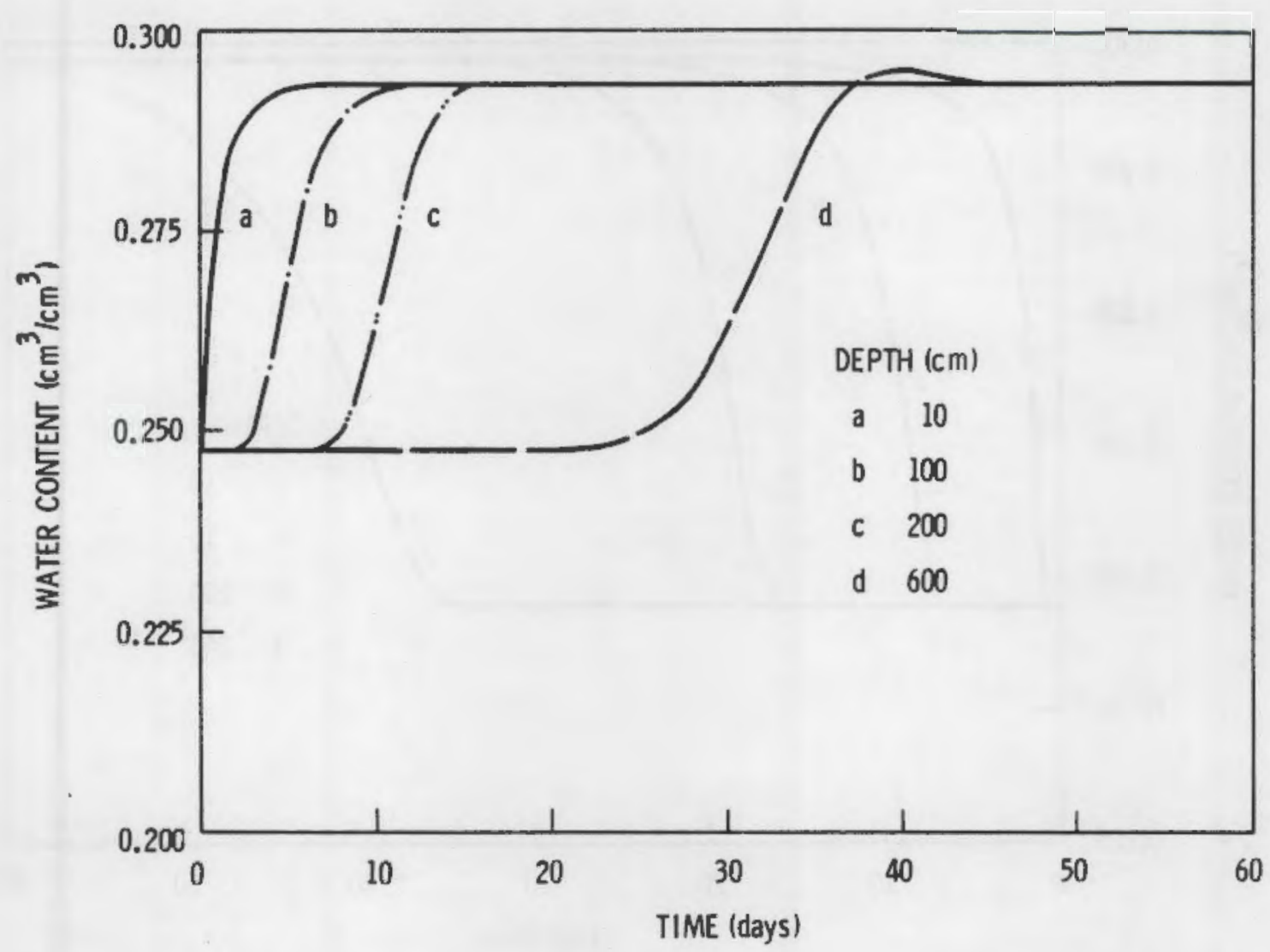

FIGURE 9. Water Content in a Medium Tailings Profile for a Constant $1 \mathrm{~cm} /$ day-Infiltration Rate Under Wet Conditions

Because of the dry conditions, a negative drainage or imbibition by the profile resulted which equaled $-7.0 \mathrm{~cm}$ of water (Figure 15). In the dry profile case, $39.62 \mathrm{~cm}$ of seepage at the $100 \mathrm{~cm}$ depth was predicted. According to the simulation, the wetting front did not reach the $400 \mathrm{~cm}$ depth in 60 days; apparentiy the front moved more slowly as water was stored in the dry profile. The greater hydraulic conductivity at the wetting front is responsible for the more rapid advance in the wet profile.

Evaporation

Evaporation is the removal of water from the soil surface by physical processes of vaporization. Energy for those processes is derived from direct solar energy (sunshine) and air turbulence (wind). Evaporation is the most complicated aspect of unsaturated flow behavior. It is a highly nonisothermal 


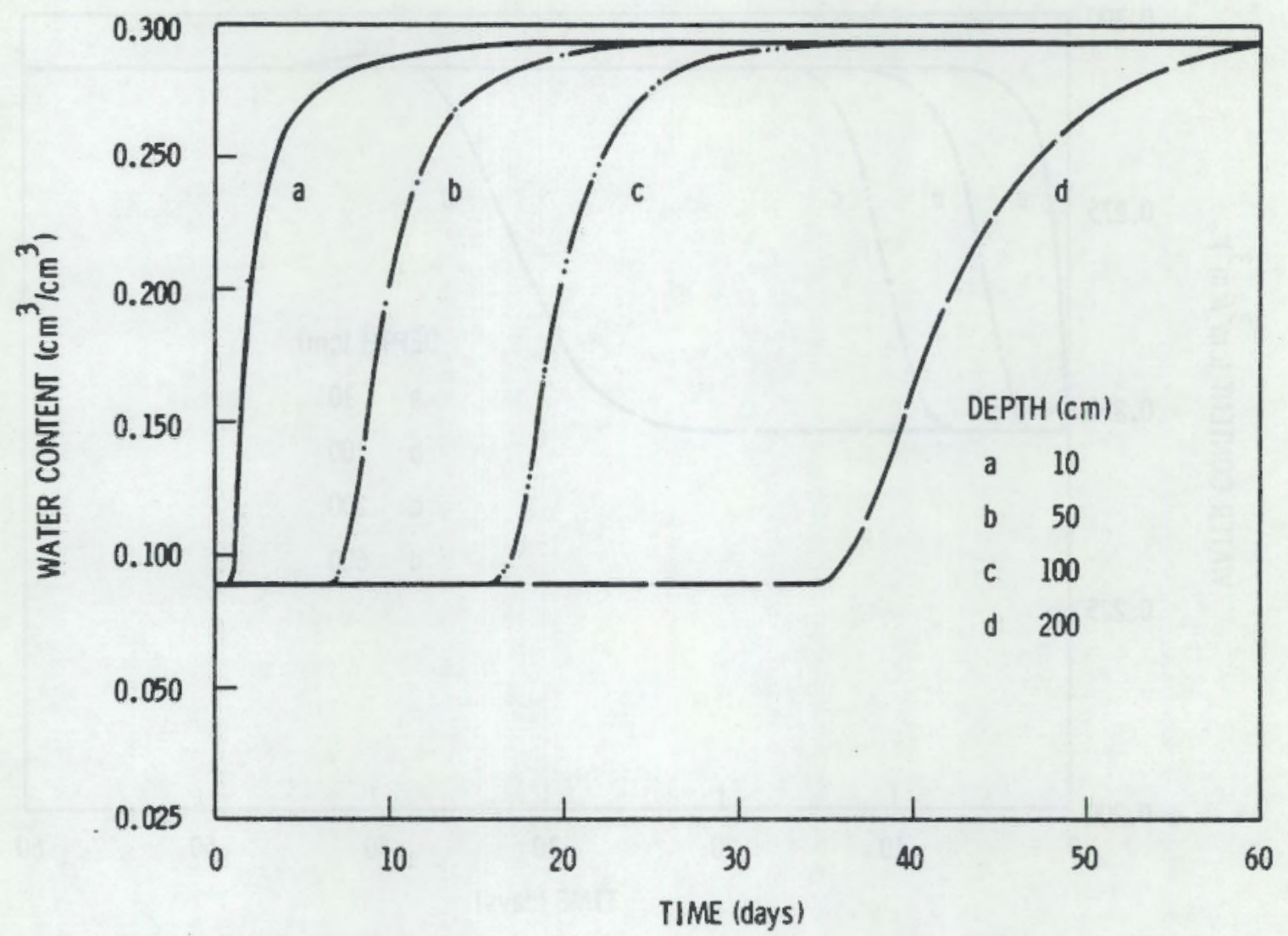

FIGURE 10. Water Content in a Medium Tailings Profile for a Constant $1 \mathrm{~cm} /$ day-Infiltration Rate Under Dry Conditions

process that can be treated only by a simplified phenomenological approach when restricted to a Darcy's law description of water flow.

Evaporation can be visualized as occurring in two stages--an energylimited and a soil-limited stage. When water is not limited, as on a nearly saturated soil surface, evaporation proceeds at a rate controlled by climatic conditions and is limited only by the availability of energy. As the soil surface dries, evaporation is limited by the hydraulic properties of the soil. In the energy-limited stage, the evaporation rate equals the potential evaporation rate estimated by various methods based on climatic measurements such as Penman's (1948). [A review of such methods was given by Nunn et al. (1970) and also by Gupta et al. (1978).] The soil-limited stage is reached when the soil surface becomes air dry and the surface-water content cannot decrease 


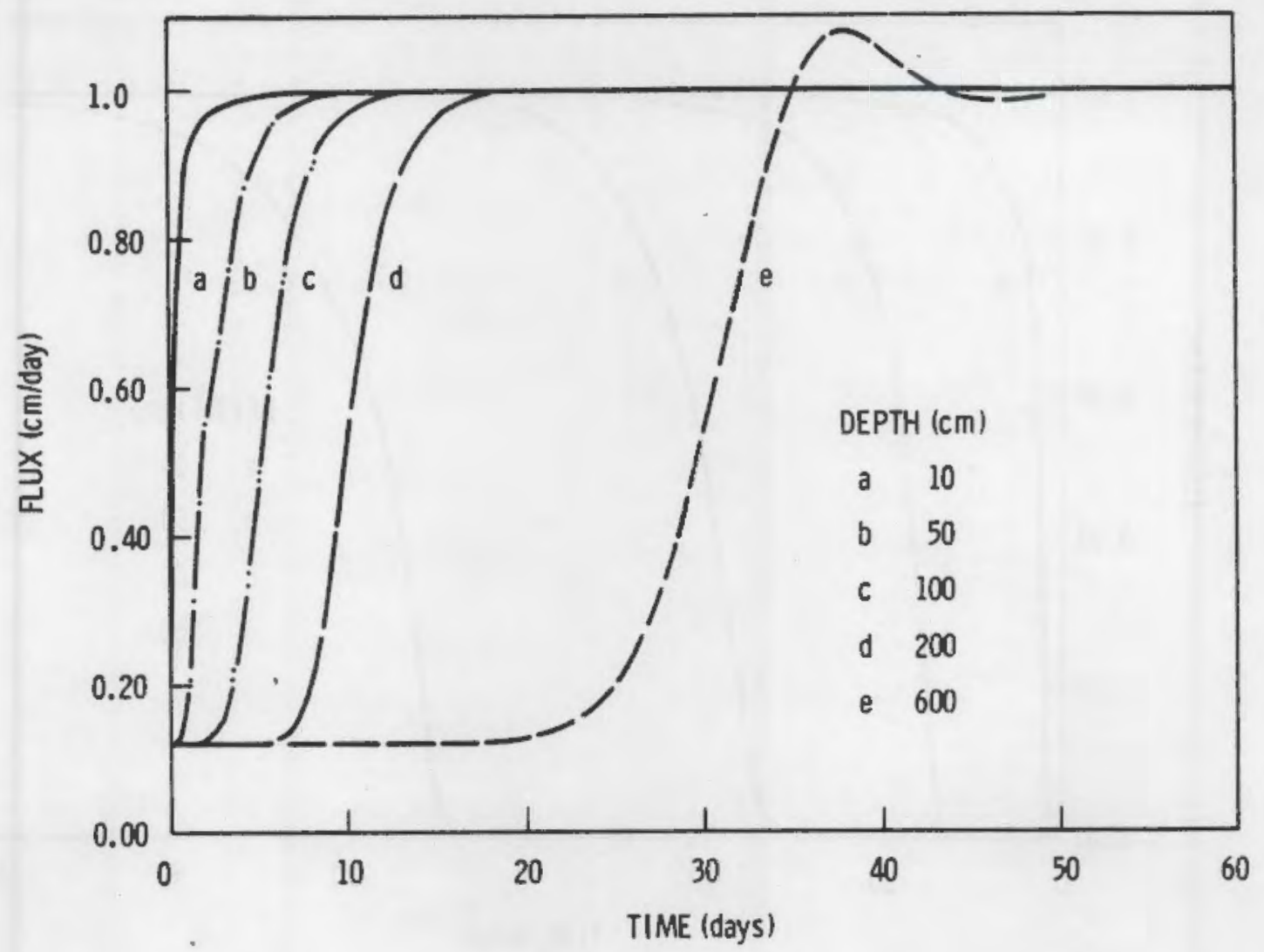

FIGURE 11. Flux at Various Depths in a Medium Tailings Profile for a Constant $1 \mathrm{~cm} /$ day-Infiltration Rate--Wet Profile Case

further. This second stage is known to satisfy a reciprocal square root of time relationship for the evaporation rate. Soll-limited evaporation is described in the model by fixing pressure head at an air-dry value when it is first attained. The second stage also includes an optional, preliminary falling rate period for which evaporation takes place as a fraction of the potential rate based on field capacity.

In some situations, a third stage of evaporation is recognized in which water vapor moves up from below a dry soil layer called a mulch (Hillel et al. 1975). Such a layer transmits water only via a vapor diffusion process. This stage (included as an option in the model) acts between the first stage and the attainment of a final air-dry condition beneath the mulch layer. 


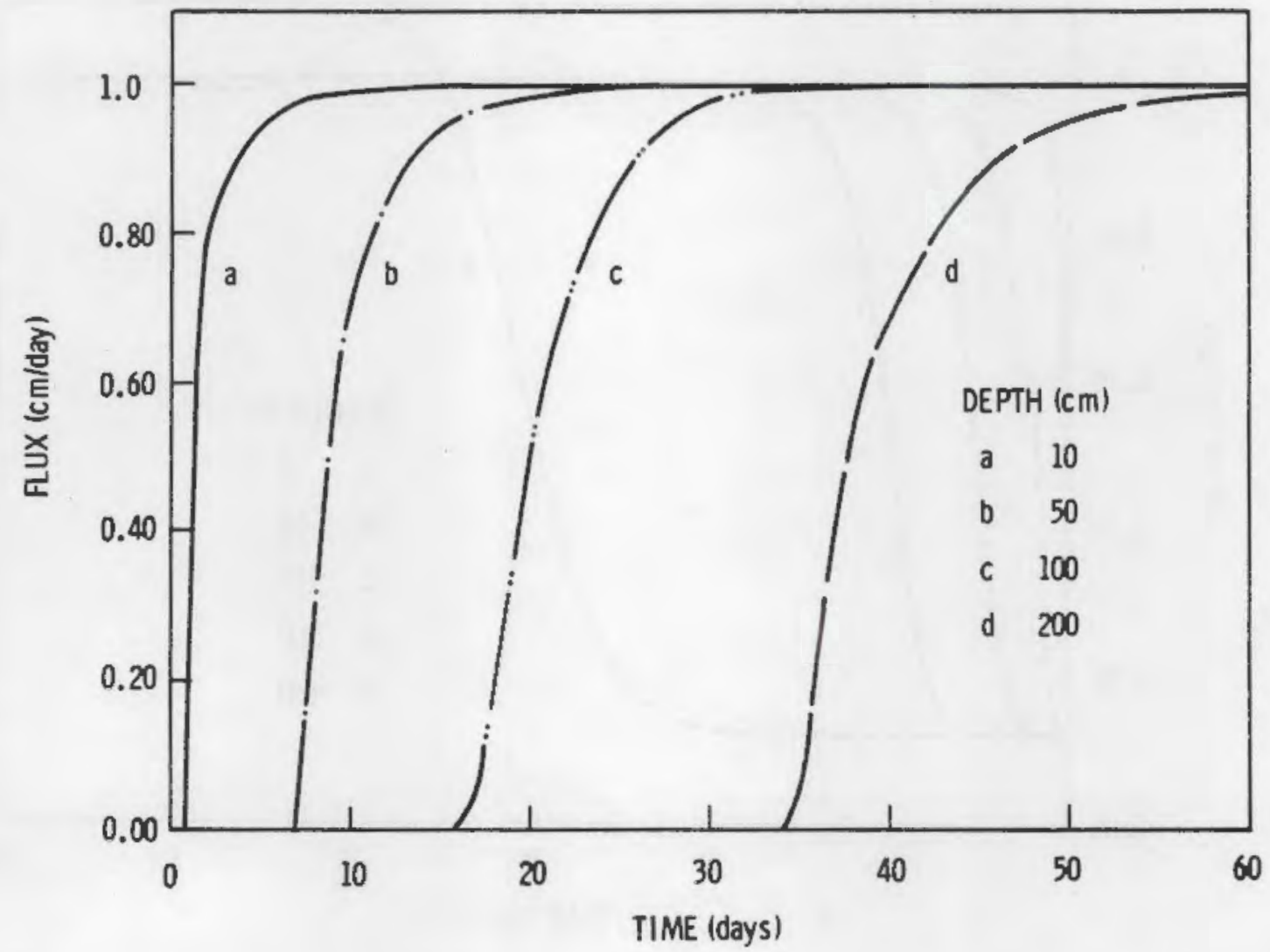

FIGURE 12. Flux at Various Depths in a Medium Tailings Profile for a Constant $1 \mathrm{~cm} /$ day-Infiltration Rate--Dry Profile Case

The simulation was performed by equating surface flux to the negative of potential evaporation rate, which equaled a constant $1 \mathrm{~cm} /$ day. The air-dry pressure-head limit was taken as $-10^{5} \mathrm{~cm}$, corresponding to e equal to 0.061 . Intermediate falling rate stages were not included in this example simulation. Profile initial conditions were the same as in the drainage case.

Figure 16 shows the water content relationship to time for the depths used in Figure 6 , which is for drainage without evaporation. Flux near the surface for 10 days is shown in Figure 17. Negative flux represents upward flux (extraction of water). Evaporation remains in the first stage about one day, since surface flux equals $-1 \mathrm{~cm} / \mathrm{day}$. After one day the flux begins to decrease in absolute magnitude as the soil dries. Drainage flux is seen to be dominant at the 100-cm depth shown in Figure 18. Flux at the compared $50-\mathrm{cm}$ depth, however, shows a reversal of flow direction. Figure 19 shows the water 


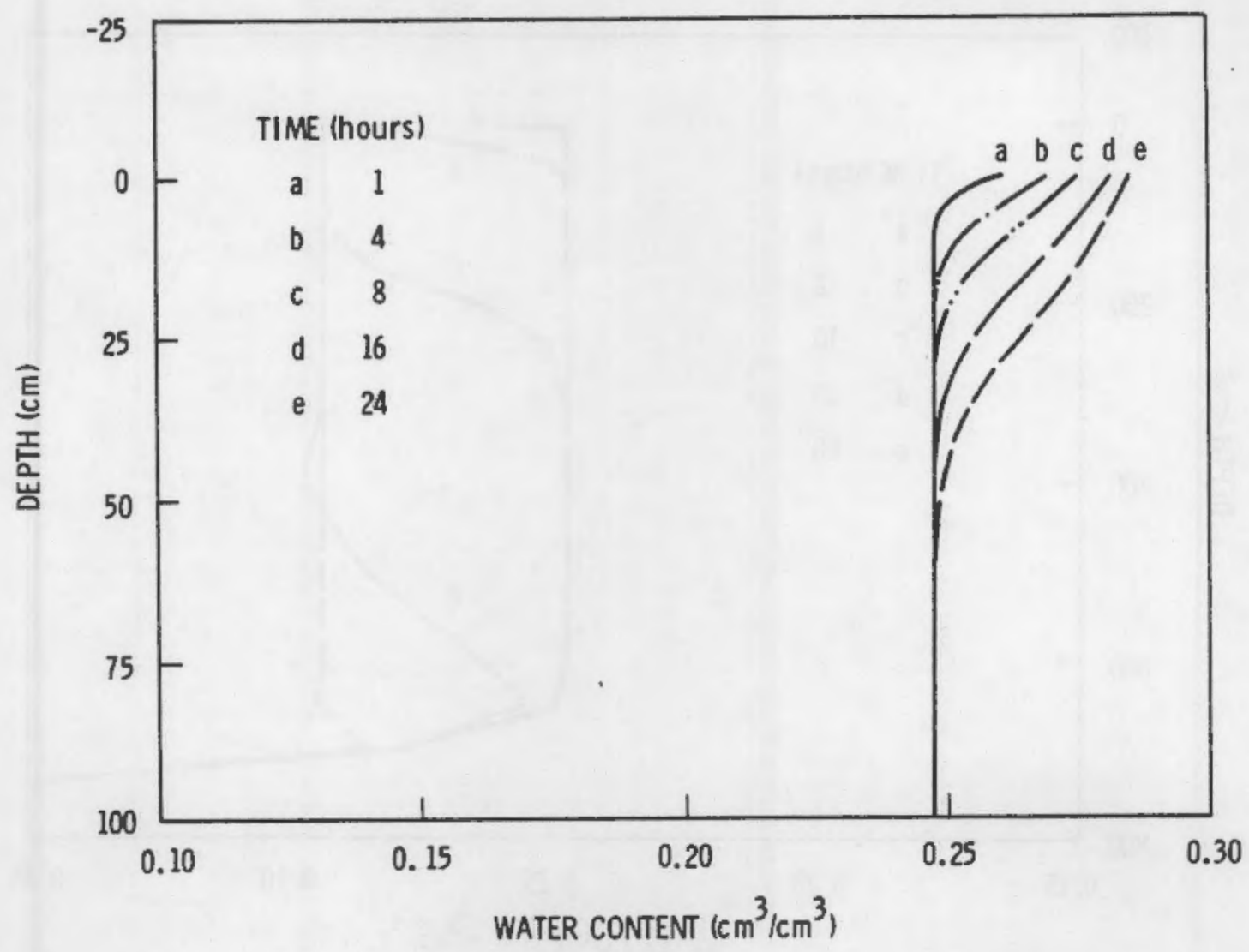

FIGURE 13. Water Content Profiles During Infiltration Under Wet Conditions for the First Day: Hours 1, 4, 8, 16, and 24

content profiles for the same days as in Figure 8 . After the first day, the minimum water content is held fixed at the surface. Profiles for the first day of evaporation are shown in Figure 20.

Final storage in the evaporation simulation is $184.7 \mathrm{~cm}$, with 3.47 of water evaporated. After $1 \mathrm{~cm}$ of water was removed on the first day, only $2.47 \mathrm{~cm}$ was removed in the following 59 days. Drainage into the water table did not differ, but seepage at the 100-cm depth reduced to $2.21 \mathrm{~cm}$, compared with $3.28 \mathrm{~cm}$ for covered drainage.

\section{SIMULATION OF THE LAYERED SOIL PROF ILE}

This section will deal with the application of basic unsaturated flow principles to layered soil profiles influenced by natural climatic conditions and growing plants. 


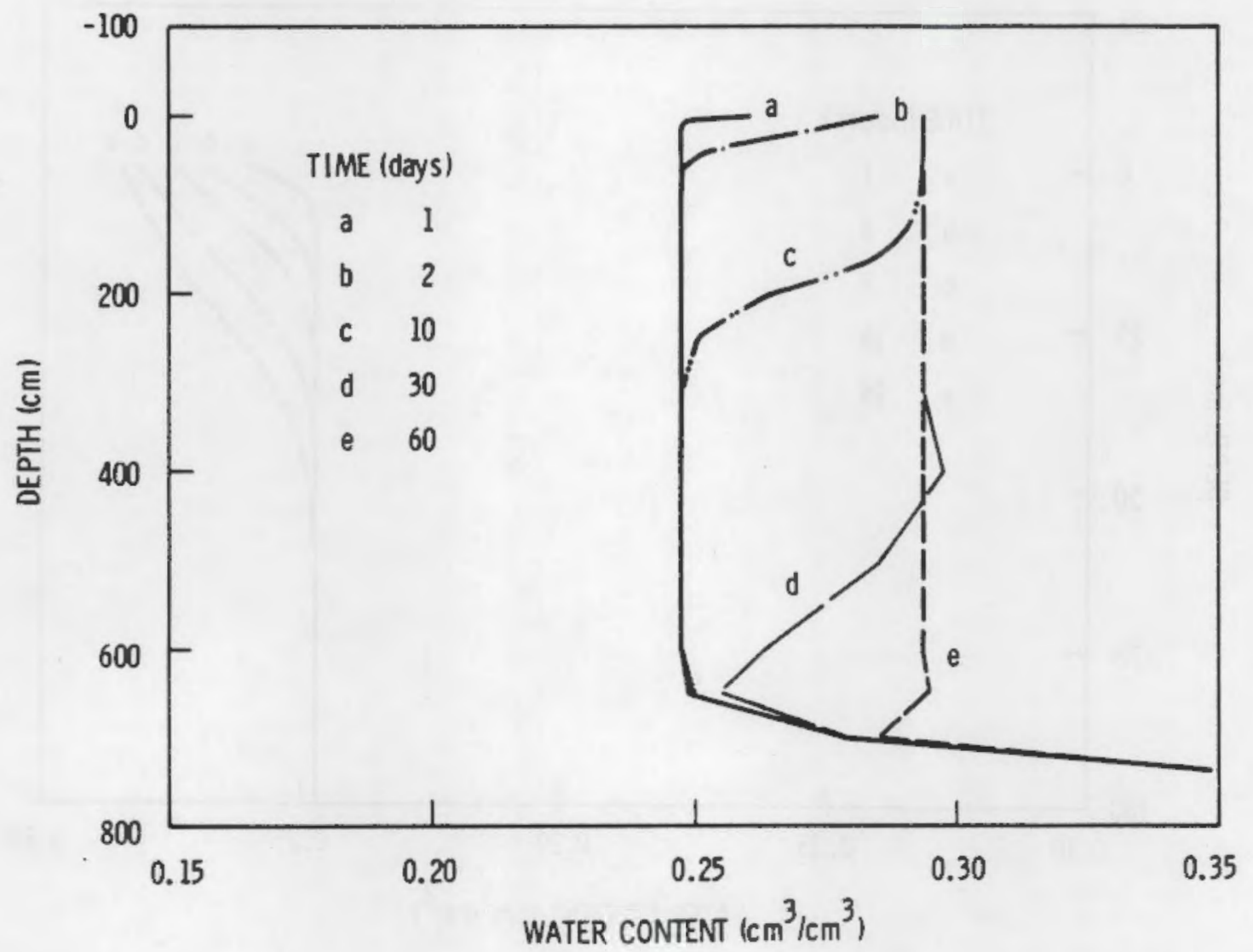

FIGURE 14. Water Content Profiles During Infiltration Under Wet Conditions: Days $1,2,10,30$, and 60

The Climate

Climatic parameters were obtained from monthly summaries of local climatological data for Grand Juntion, Colorado, published by the U.S. Department of Commerce, National Climatic Center, Asheville, NC. The data used from the sumnaries for the years 1976 and 1979 were the following:

- hourly precipitation (inches)

- temperature $\left({ }^{\circ} \mathrm{F}\right)$--daily maximum, minimum, average, and average dew point

- average wind speed (m.p.h.)

- percent of possible sunshine and sky cover in tenths

- maximum and minimum relative humidity. 


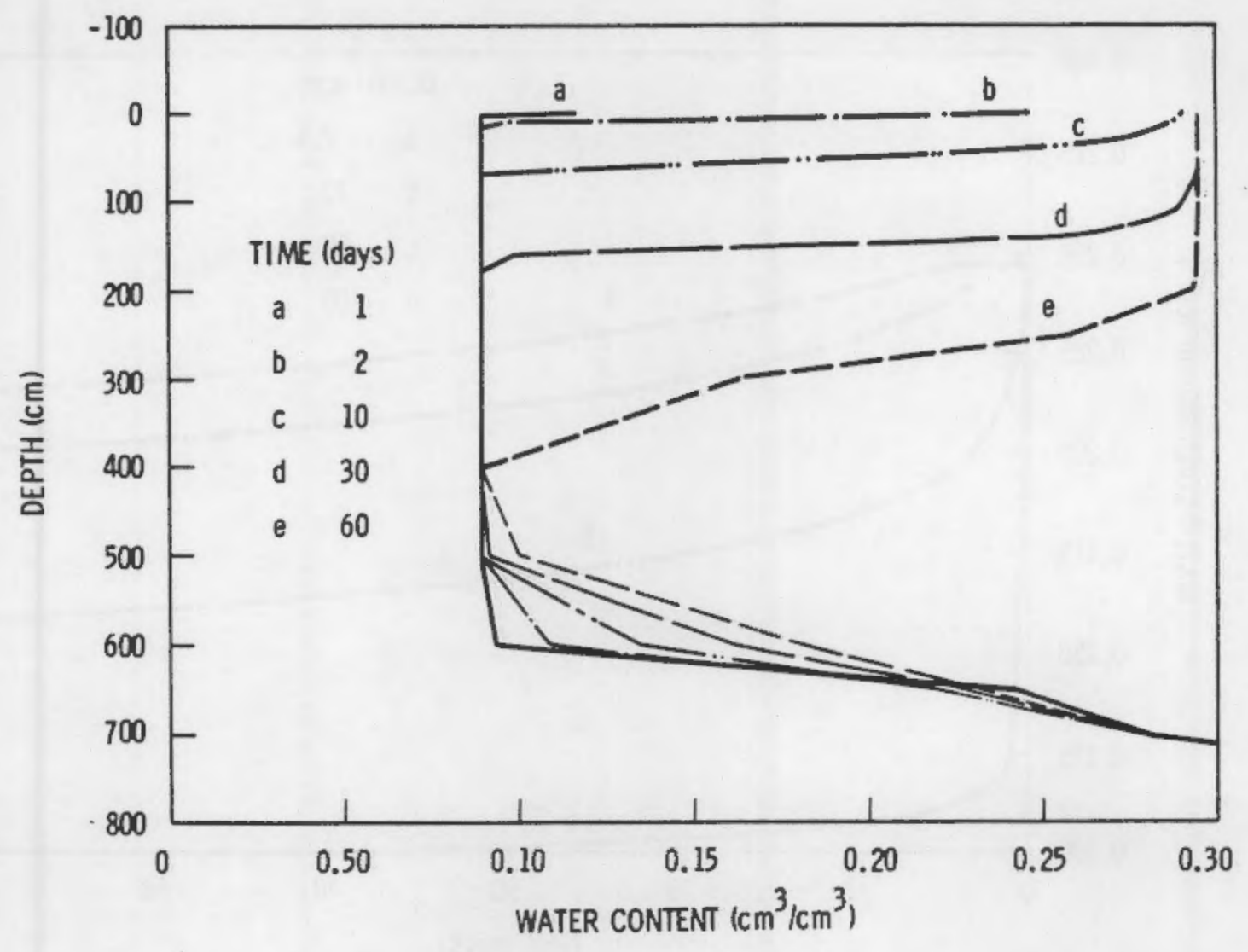

FIGURE 15. Water Content Profiles During Infiltration Under Dry Conditions: Days $1,2,10,30$, and 60

Hourly precipitation determined the modeled water infiltration rate. Other climatic parameters were used to estimate potential evapotranspiration on a daily basis.

\section{Potential Evapotranspiration}

The amount of water evaporated from a soil surface and transpired by plants each day is called the evapotranspiration rate, denoted ET. Potential evapotranspiration, denoted PET, is the evapotranspiration when water is not limited (a free water surface), and it represents the maximum evaporation rate possible for given climatic conditions. A solar energy budget equation determines the ET:

$$
R_{n}=H+L E T-G
$$




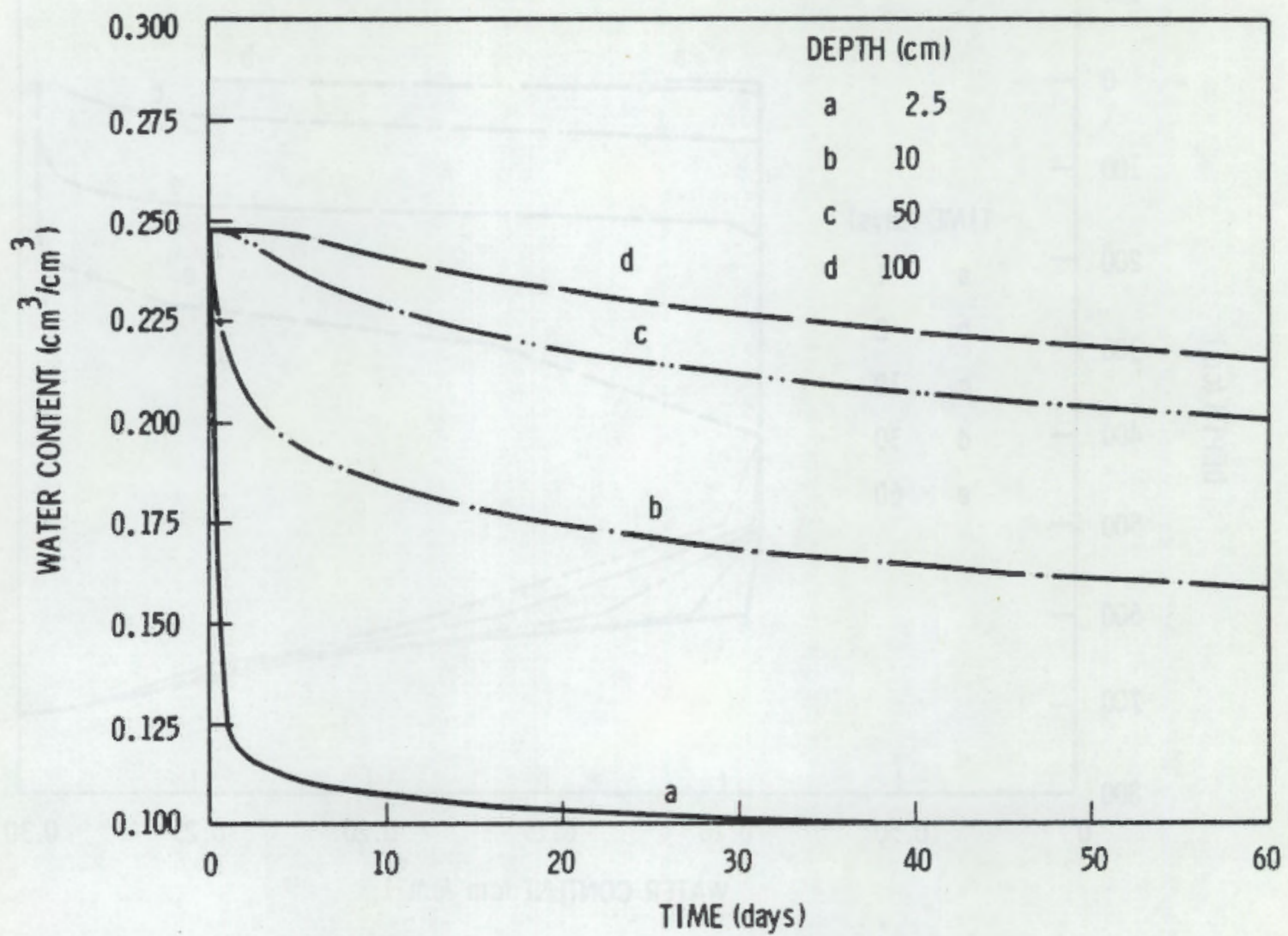

FIGURE 16. Water Content in a Medium Tailings Profile for Evaporation Conditions: Depths 2.5,10,50, and $100 \mathrm{~cm}$

where $R_{n}$ is net radiation, $H$ is energy utilized in heating the air, $G$ is energy utilized in heating the soil, and $L$ is the latent heat of vaporization $(585 \mathrm{cal} / \mathrm{g})$. The net radiation, $R_{n}$, is related to the global radiation, $R_{s}$, arriving at the surface and the net longwave radiation, $R_{f}$, by the equation

$$
R_{n}=R_{S}(1-\varepsilon)+R_{1}
$$

where $\varepsilon$ is the albedo (fraction of global radiation reflected at the surface). Radiations are in units of calories per square centimeter per day so that ET can be expressed in $\mathrm{cm} /$ day of water.

The Penman method based on a combination of energy balance and vapor mass transfer methods was used to estimte PET. The equation is 


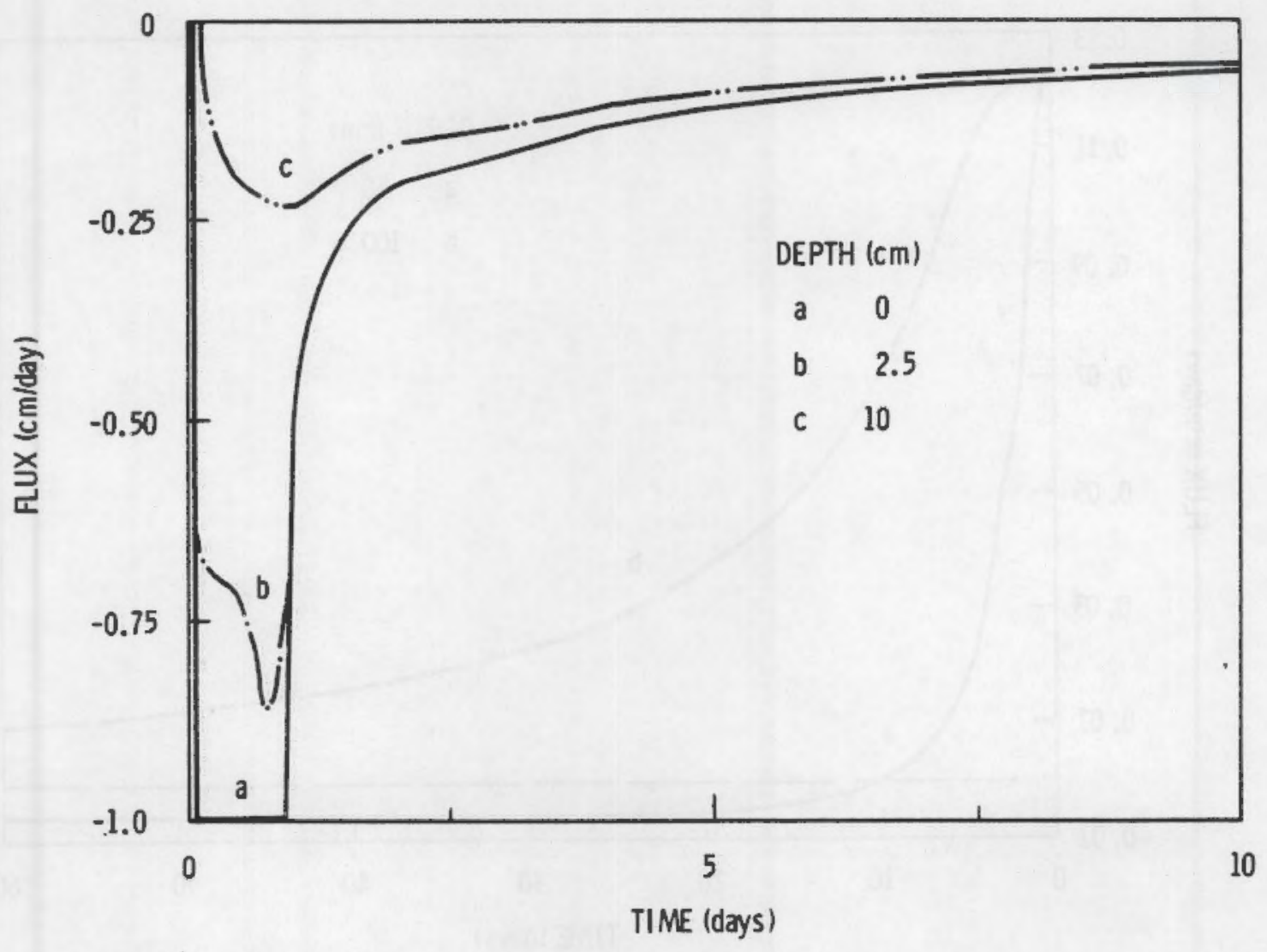

FIGURE 17. Flux Near the Surface of a Medium Tailings Profile for Evaporation Conditions: Depths $0,2.5$, and $10 \mathrm{~cm}$

$$
P E T=W R_{n}+(1-W) f(U)\left(p_{s}-p_{a}\right)
$$

where

$$
\begin{aligned}
W & =\Delta / \Delta+\gamma \\
f(U) & =0.27(1+U / 100)
\end{aligned}
$$

with

$$
\begin{aligned}
\Delta= & \text { siope of the saturated vapor pressure-temperature curve } \\
& \left(\mathrm{mb} /{ }^{\circ} \mathrm{C}\right) \\
\gamma= & \text { psychrometric constant }\left(\mathrm{mb} /{ }^{\circ} \mathrm{C}\right) \\
f(U)= & \text { wind transfer function } \\
U= & \text { daily wind run }(\mathrm{km} / \text { day) at } 2-m \text { height }
\end{aligned}
$$




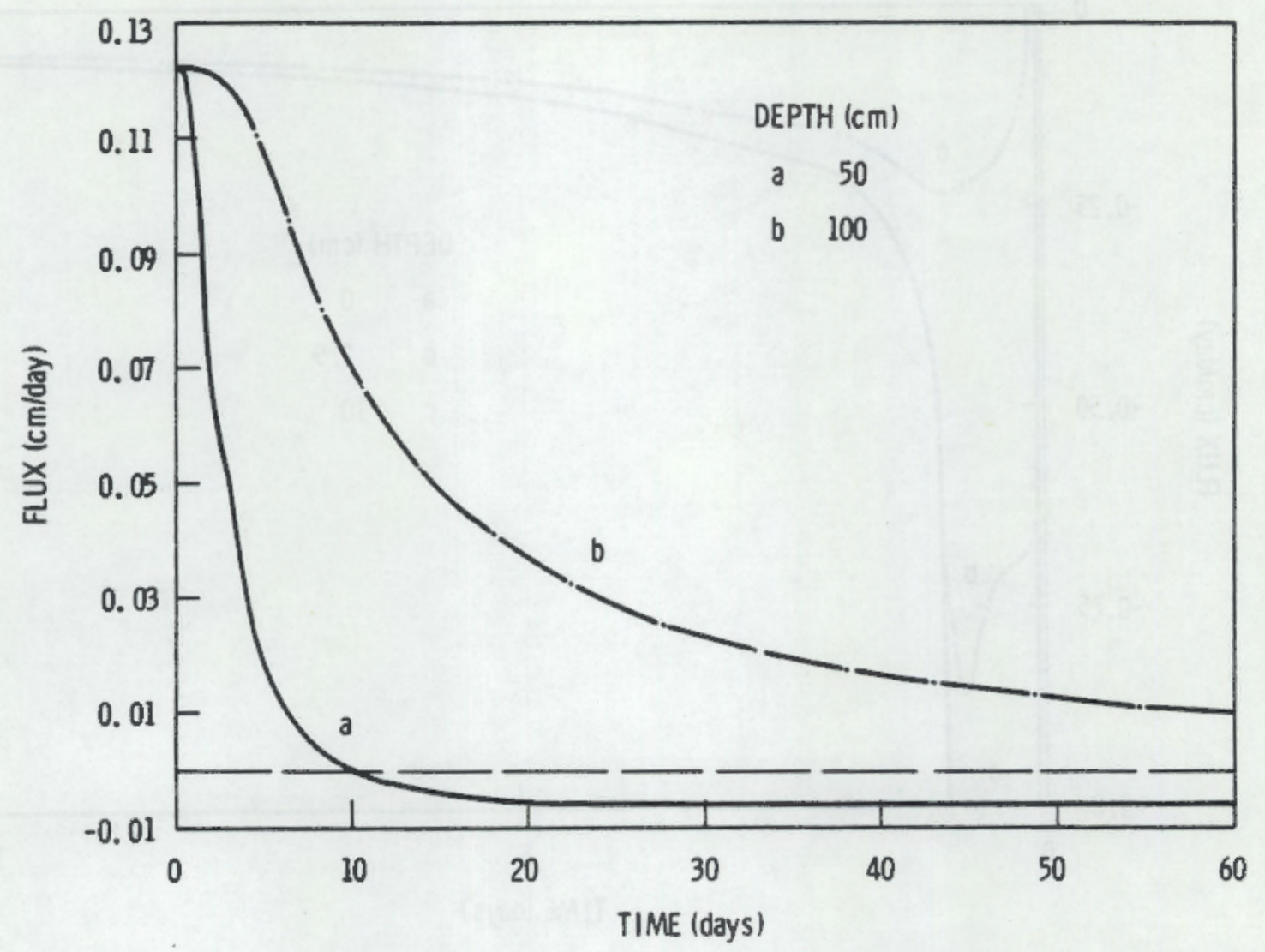

FIGURE 18. Flux in the Medium Tailings Profile for Evaporation Conditions: Depths 50 and $100 \mathrm{~cm}$

$p=$ vapor pressure $(\mathrm{mb}) ; \underline{s}$ denotes the saturated value, and $\underline{a}$ denotes the actual for the air at the daily dew point temperature.

$R_{n}=$ net radiation $(\mathrm{mm} /$ day) in equivalent unit of water.

Parameters for Equation (19) were estimated from climatic data as follows:

$$
R_{n}=0.75 R_{s}-f(T) f\left(p_{a}\right) f(n / N)
$$

where

$$
\begin{aligned}
f(T) & =\left(2.0 \times 10^{-9}\right) T^{4} \\
f(P) & =0.34-0.044 \sqrt{P} \\
f(n / N) & =0.1+0.9 \mathrm{n} / \mathrm{N}
\end{aligned}
$$




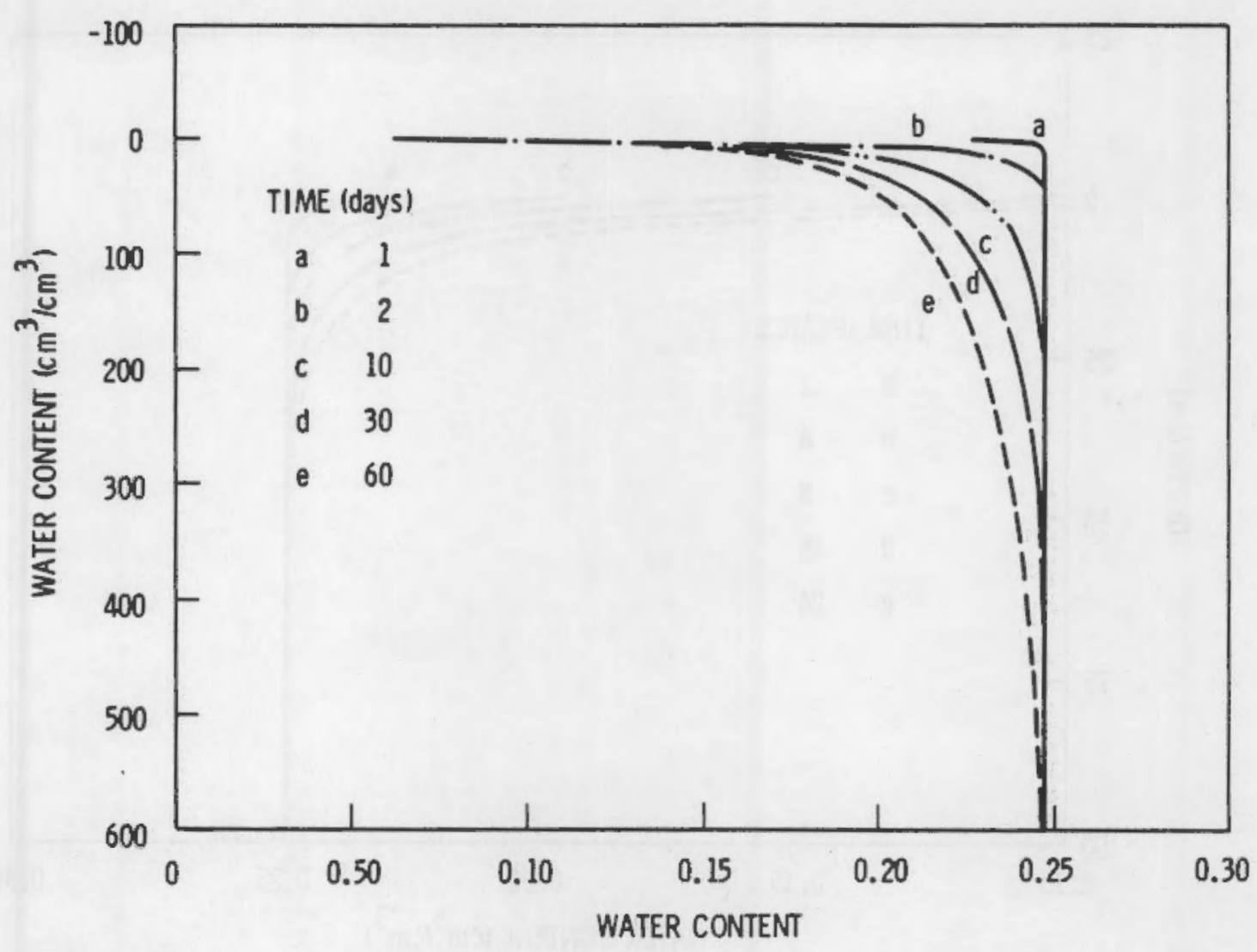

FIGURE 19. Water Content Profiles at Various Times During Evaporation from Medium Tailings: Days 1, 2, 10, 30, and 60

with

$$
\begin{aligned}
T & =\text { temperature }\left({ }^{\circ} \mathrm{K}\right) \text { mean value } \\
n / \mathrm{N} & =\text { ratio of actual sunshine hours to possible }
\end{aligned}
$$

and

with

$$
\begin{aligned}
R_{s} & =(0.25+0.5 \mathrm{n} / \mathrm{N}) R_{a} \\
r & =0.0006595 P_{m b} \\
\Delta & =\left(p_{s} / T\right)(6790.5 / T-5.028) \\
P_{s} & =\exp (54.88-6790.5 / T-5.028 \ln T) \\
P_{a} & =R H_{\text {mean }} P_{s} / 100
\end{aligned}
$$

$$
\begin{aligned}
\mathrm{RH}_{\text {mean }} & =\text { mean relative humidity }(\%) \\
\mathrm{P}_{\mathrm{mb}} & =\text { atmospheric pressure }(\mathrm{mb}) \\
\mathrm{R}_{\mathrm{a}} & =\text { extraterrestrial radiation (mm/day) at given location. }
\end{aligned}
$$




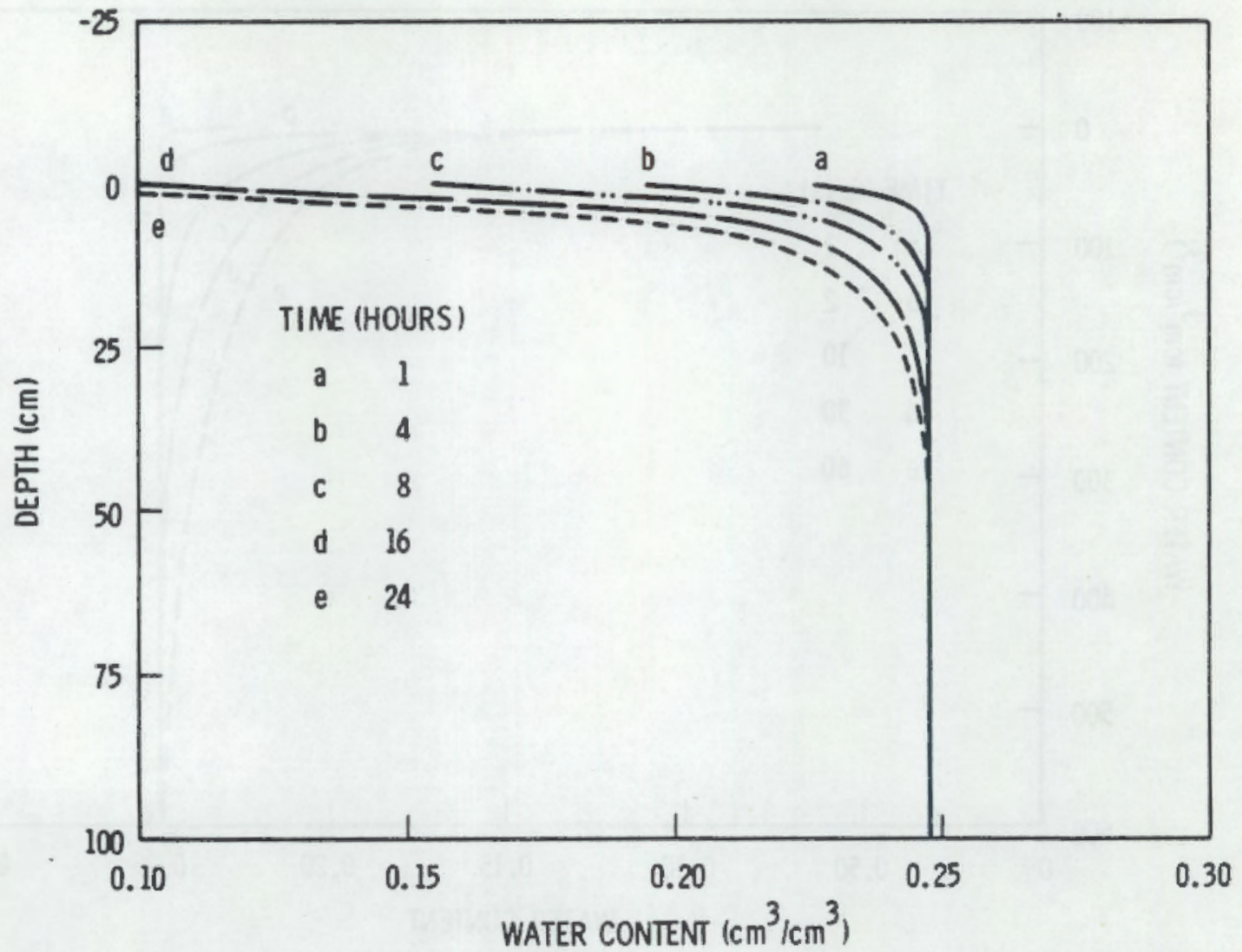

FIGURE 20. Water Content Profiles Near the Surface for the First Day of Evaporation from Medium Tailings

The FAOPET computer program, which includes a number of other methods, was used to compute Equation (19) (Gupta et al. 1978). Values for $R_{a}$ measured as monthly averages over the earth are tabulated in the FAOPET program.

\section{Actual Modeled Evaporation}

To model actual evaporation, the two main energy-limited and soil-limited stages are subdivided into three stages. As previously discussed, evaporation can proceed at the potential maximum rate only when the soil surface is nearly saturated. During drying the actual evapotranspiration is given by the Thornthwaite-Mather method:

$$
E T=\left(\frac{\bar{\theta}-\theta_{W}}{\theta_{f c}^{-} \theta_{W}}\right) \text { PET }
$$


where $\theta_{f C}$ is a field capacity water content and $\theta_{w}$ is a wilting-point lower limit. Equation (20) is evaluated for an average water content $\theta$ taken over a specified storage depth defining the $\theta_{\mathrm{fc}}$. When no plants are present, Equation (20) estimates only evaporation. If the soil surface dries below a certain limit pressure head, $-h_{\text {surf }}$, then evaporation $E$ is modeled as a vapor diffusion process through a mulch layer given by

$$
\begin{aligned}
& \qquad E={ }_{B D_{a}}(b-\theta)\left(\rho_{\text {air }}-\rho_{\text {soi } 1}\right) / \Delta Z \\
& D_{a}=\text { vapor diffusivity in air }\left(0.24 \mathrm{~cm}^{2} / \mathrm{sec}\right) \\
& B=\text { enhancement factor }(2.0) \\
& b=\text { total porosity }\left(\mathrm{cm}^{3} / \mathrm{cm}^{3}\right) \\
& D=\text { vapor density }\left(\mathrm{g} / \mathrm{cm}^{3}\right) \\
& \Delta Z=\text { hydrophobic layer thickness }(\mathrm{cm}) .
\end{aligned}
$$

The vapor densities in air and soil include the dependence on different temperatures of the air and soil, and can be related to the respective vapor pressures by using the ideal gas law. Vapor pressure in the soil surface, which is nearly saturated until the soil becomes very dry, is calculated from the isotherm Equation (6).

In the last drying stage, the pressure head attains its lowest air-dry value, and then the simulation continues with that value held as a surface boundary condition. The first two stages of evaporation, however, are determined by Equations (20) and (21) as surface flux boundary conditions. These three stages of drying result in an estimate of evaporation compatible with soil-water availability based on the hydraulic properties.

Plant Roots Sink Model

Various models for the plant root sink term $S(z, t)$ appearing in Equation (3) have been summarized by Gupta et al. (1978). A model based on a modification of Feddes' (1978) root water extraction model was selected. The sink function has the form

$$
S(z, \theta)=S_{\max } r(z) a(\theta)
$$


where

$$
a(\theta)=\left\{\begin{array}{l}
0 \text { if } \theta \leq \theta_{W} \\
\left(\theta-\theta_{W}\right) /\left(\theta_{d}-\theta_{W}\right) \text { if } \theta_{w} \leq \theta \leq \theta_{d} \\
1 \text { if } \theta_{d} \leq \theta \leq \theta_{\text {an }} \\
0 \text { if } \theta>\theta_{\text {an }}
\end{array}\right.
$$

The values $\theta_{\mathrm{w}}<\theta_{\mathrm{d}}<\theta_{\mathrm{an}}<\theta_{\mathrm{S}}$ are various water content limits associated with root uptake activity, and $r(z)$ is the root density distribution function which changes in time as the roots grow. Under maximum transpiration conditions, $\theta_{\mathrm{d}} \leq \theta^{*} \leq \theta_{\text {an }}$, $S_{\max }$ satisfies

$$
\begin{aligned}
\text { PT } & =\int_{0}^{z m} S\left(z, \theta^{\star}\right) d z=S_{\max } \int_{0}^{z m} r(z) d z \\
& =S_{\max }
\end{aligned}
$$

where PT is the potential transpiration.

Then

$$
T=\int_{0}^{z} S(z, \theta) d z \leq P T
$$

determines actual transpiration, as less than the potential rate. The root density function was described by

$$
r(z)=a e^{-b z}
$$

where

$$
a=b /\left(1-e^{-b z}\right) \quad\left(c m^{-1}\right)
$$

and $z_{m}$ is the maximum root depth. Parameters $z_{m}$ and a depend on growth time. In cases when the potential transpiration must equal the actual, an 
activity factor $A$ is introduced so that the sink function Equation (22) with $S_{\max }=A P T$ yields the maximum at each time. This option allows the model to match a known transpiration, rather than estimate it as less than some poteritial value.

Parameters for the root density function are evaluated by fitting root growth data in terms of root length or root weight density. For this application, $a$ and $b$ were estimated from root weight data for cheatgrass penetrating to the depth of $120 \mathrm{~cm}$. Values are $a=1.0$ and $b=0.034$. The normalization parameter, $a$, is re-evaluated at each time during the simulation, and $b$ is taken as time independent. Water content limits for the simulation were $\theta_{a n}=$ $0.391(\mathrm{~h}=5 \mathrm{~cm}), \theta_{d}=0.188(\mathrm{~h}=1000 \mathrm{~cm})$, and $\theta_{\mathrm{w}}=0.113(\mathrm{~h}=20000 \mathrm{~cm})$. These are values selected to demonstrate functioning of the model and do not necessarily represent cheatgrass. A short spring growing season of 70 days was assumed for the hypothetical grass plant, and the maximum root depth at various times after beginning growth is given in Table 3 .

Plant growth began on Day 120 for the simulations. A fixed activity of $A=2$ was used in simulations. The plant sink model is considerably oversimplified since growth dependence on available soil water is not taken into account. Addition of such a dependence, however, would cause no fundamental probiems.

TABLE 3. Days Required for Plant Roots to Reach Various Depths

$\begin{array}{ccccc}\frac{\text { Depth }(\mathrm{cm})}{0.0} & \frac{\text { Days }}{5} & & \frac{\text { Depth (cm) }}{30} & \frac{\text { Days }}{20} \\ 2.5 & 6 & 35 & 25 \\ 5.0 & 7 & 40 & 35 \\ 7.5 & 8 & 50 & 40 \\ 10 & 10 & 60 & 50 \\ 15 & 11 & 70 & 60 \\ 20 & 15 & 80 & 70 \\ 25 & 16 & & \end{array}$


Partitioning Evapotranspiration

To apply the evaporation and plant root sink models in a simulation of climatic conditions, the evapotranspiration must be divided into its component parts as expressed by the equation

$$
P E T=P E+P T
$$

In developing the UNSAT model, Gupta et al. (1978) reviewed a number of models for partitioning PET into PE and PT based on the amount of leaf canopy cover and leaf area index. These test simulations, however, employed specific experimental observations for cheatgrass (Hinds 1975). Hinds found a partitioning of PET as a function of growing time described approximately as follows:

$$
P T=(c(t-40)+0.42) P E T
$$

where

$$
c=0.0013 \text { if } t \leq 40 \text { and } c=-0.0035 \text { if } t>40 \text {, and } P T=0 \text { if } t<5 \text { or }
$$

$t>70$. The partitioning applied to north exposure of cheatgrass, whereas south exposure gave a somewhat different relationship. PT was estimated from Equation (25) using the Penman calculation for PET. Actual transpiration was obtained from Equations (22) and (23). Potential evaporation (PE) from the soil surface was estimated from Equations (24) and (25), and Equation (20) was assumed to hold for estimating actual evaporation $E$ from $P E$ when $\mathrm{h} \leq 1000 \mathrm{~cm}$. Equation (21) was applied when $h>1000 \mathrm{~cm}$, and third-stage drying began when $\mathrm{h}$ reached $10^{5} \mathrm{~cm}$. Using a value for $\mathrm{h}_{\text {surf }}$ other than $1000 \mathrm{~cm}$ (but near wet conditions) resulted in only small changes in simulation estimates of actual evaporation. A change in root activity $A$ from 1 to 2 increased transpiration by about $10 \%$.

\section{Example Simulation Results}

Some simulation results for three- and four-layer soil profiles will be described. Climatic conditions for 1976 and 1979 were used, but only the 1979 
year will be discussed in detail. The three-layer profile consisted of $130 \mathrm{~cm}$ of Grand Junction clay soil (layer 1 ), $15 \mathrm{~cm}$ of clay/gravel mix (layer 2), and medium tailings from the $145-\mathrm{cm}$ to the 763-cm depth (layer 3 ). The four-layer profile had $30 \mathrm{~cm}$ of clean rock replacing Grand Junction clay between the $100 \mathrm{~cm}$ and $130 \mathrm{~m}$ depths (Table 2). Initial conditions in the profiles represent near-equilibrium conditions below the clay/gravel layer for the $763-\mathrm{cm}$ deep water table. Moisture conditions above the clay/gravel layer are established by repetitions of simulations with the recorded climatic conditions, and could represent initital conditions established by any unknown previous climatic history. The clay/gravel layer, in which we wish to study soil-water retention, however, is initialized as if inserted into the profile with a nearly saturated state.

Climate for 1976 had $13.5 \mathrm{~cm}$ of rainfall and $185.1 \mathrm{~cm}$ of potential evapotranspiration, and $1979 \mathrm{had} 22.6 \mathrm{~cm}$ of rainfall and $178.3 \mathrm{~cm}$ of PET. Under the wetter conditions, 1979 actually showed lower PET. The climate and PET pattern for Grand Junction, Colorado, is somewhat similar to that for Hanford Station, Washington (Gee and Simmons 1979).

Three-Layer Profile

The three-layer soil profile was simulated with and without a plant root sink for purposes of comparison. Results on water content versus time for selected depths are shown in Figures 21 and 22 . At the surface, water content closely follows the influence of climatic conditions, with sharp peaks indicating rainfall events. Water content at the 20 - and $80-\mathrm{cm}$ depths is substantially reduced when plants are present (Figure 22). In the clay/gravel layer at 140-cm depth, water content shows a drainage type decrease for 60 days, and thereafter is nearly constant. Flat portions of the surface curves between 180 and 300 days indicate that the minimum air-dry water content was reached. Minimum values represent the water content just below the mulch layer, which was taken as $1 \mathrm{~cm}$ thick.

Cumulative flux or seepage at various depths is shown in Figures 23 and 24. The sharp peaks on the surface curve indicate rainfall events and the decreasing values indicate evaporation. The effects of the plants begin at 


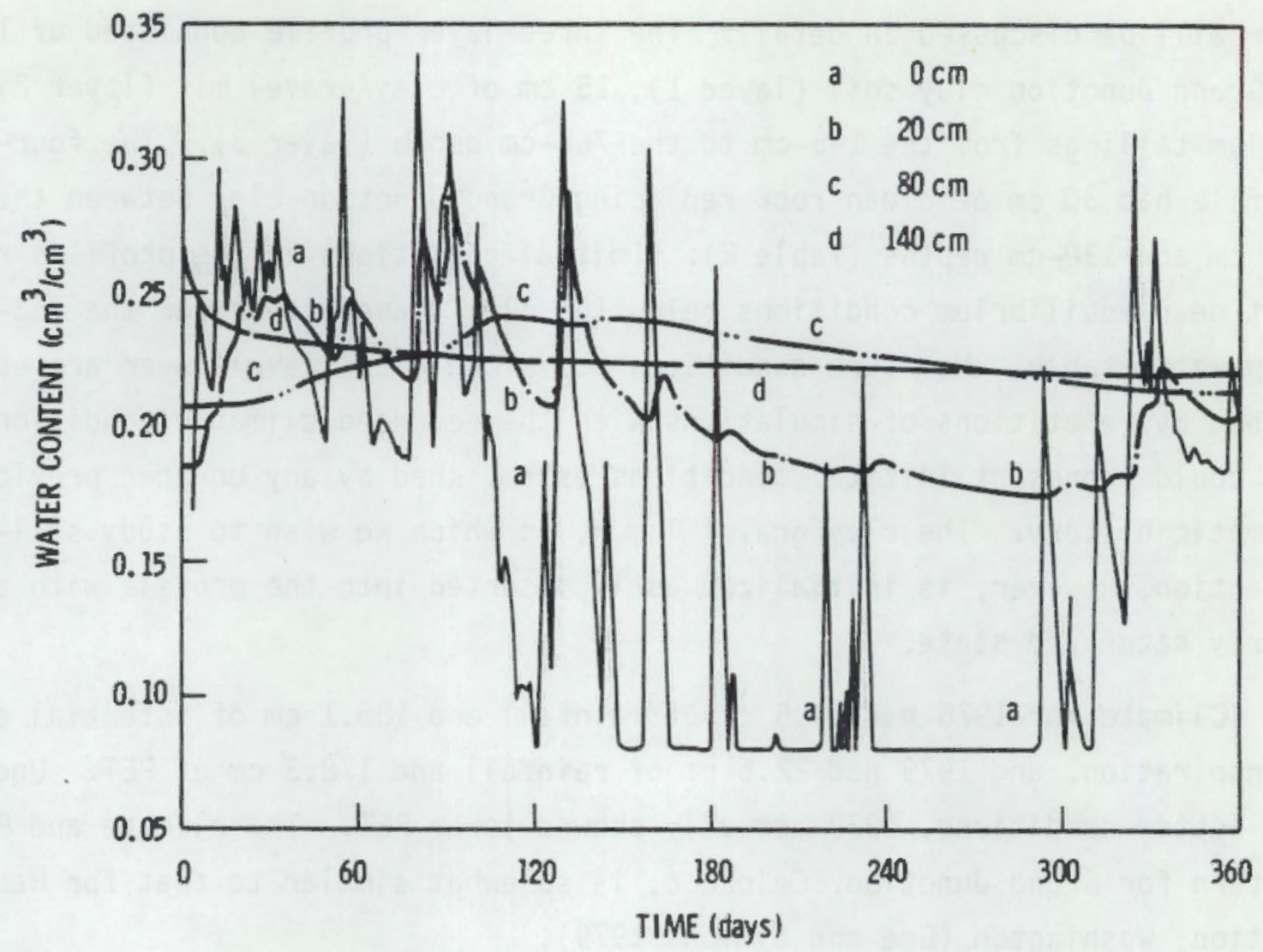

FIGURE 21. Water Content in the Three-Layer Profile for the $1979 \mathrm{Cl}$ imate

120 days. A distinct difference in the effect of plants is seen in these graphs. Figure 24 shows a continued increase in surface seepage (infiltration), because more water leaves the profiles through transpiration than by evaporation (Figure 23). Seepage in the clay/gravel layer is essentially zero. An upward movement of water at the $80-\mathrm{cm}$ depth after plants stop transpiring is aiso seen in Figure 24. Apparentiy, water is moving to balance the storage deficit created in the root zone. This difference in soil-water storage from the surface to the $80-\mathrm{cm}$ depth is displayed by Figure 25 .

Water content profiles for various days, including the plant growth season, are shown in Figures 26 and 27 . A distinct difference in profiles occurs on Day 150. Little change takes place in the clay/gravel layer over the indicated period. 


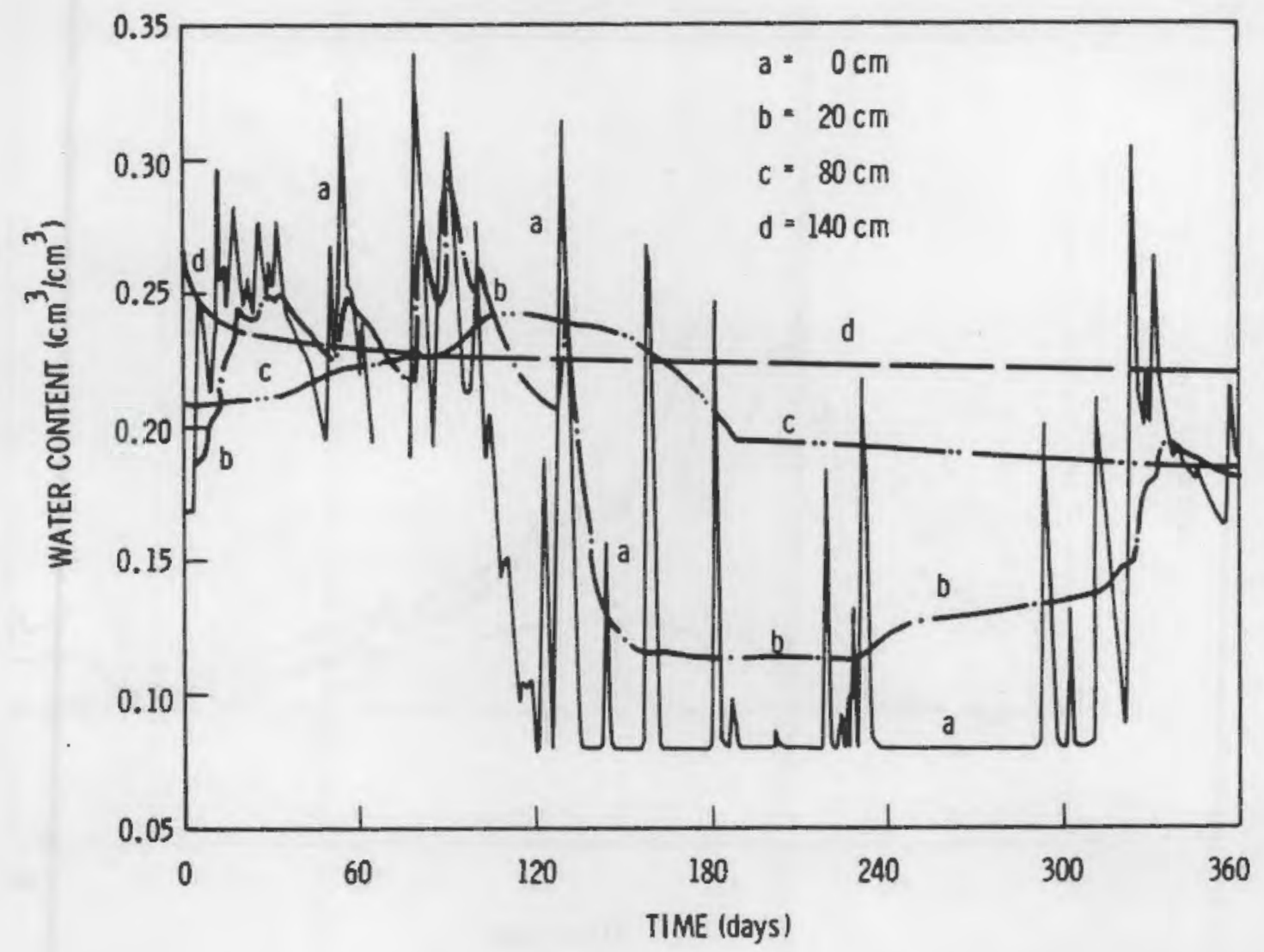

FIGURE 22. Water Content in the Three-Layer Profile with Piants for the 1979 Climate

The root uptake rate pattern is shown in Figure 28 , and total transpiration during the growth season is given in Figure 29. Response of the root sink to varying climatic conditions is clearly seen. Water balance in the profile with and without plants is given in Table 4.

Infiltrations calculated by the model differed from actual rainfall as a result of numerical errors associated with the time steps utilized. However, in general, the profile lost water over the year when plants were present. The additional $0.5-\mathrm{cm}$ infiltration in bare soil can be subtracted from the storage to make comparison with equal inputs. Hinds (1975) measured evaporation at about $4 \mathrm{~cm}$ and transpiration at about $7 \mathrm{~cm}$ during the growing season, whereas the model gave 2.1-cm of evaporation. Another run using $\mathrm{h}_{\text {surf }}=$ $15000 \mathrm{~cm}$ and $A=1$ gave an evaporation value of $2.8 \mathrm{~cm}$ and transpiration of $8.2 \mathrm{~cm}$ for the growing season. It is important to remember that the climates, however, are not exactly the same for the measured and modeled results. 


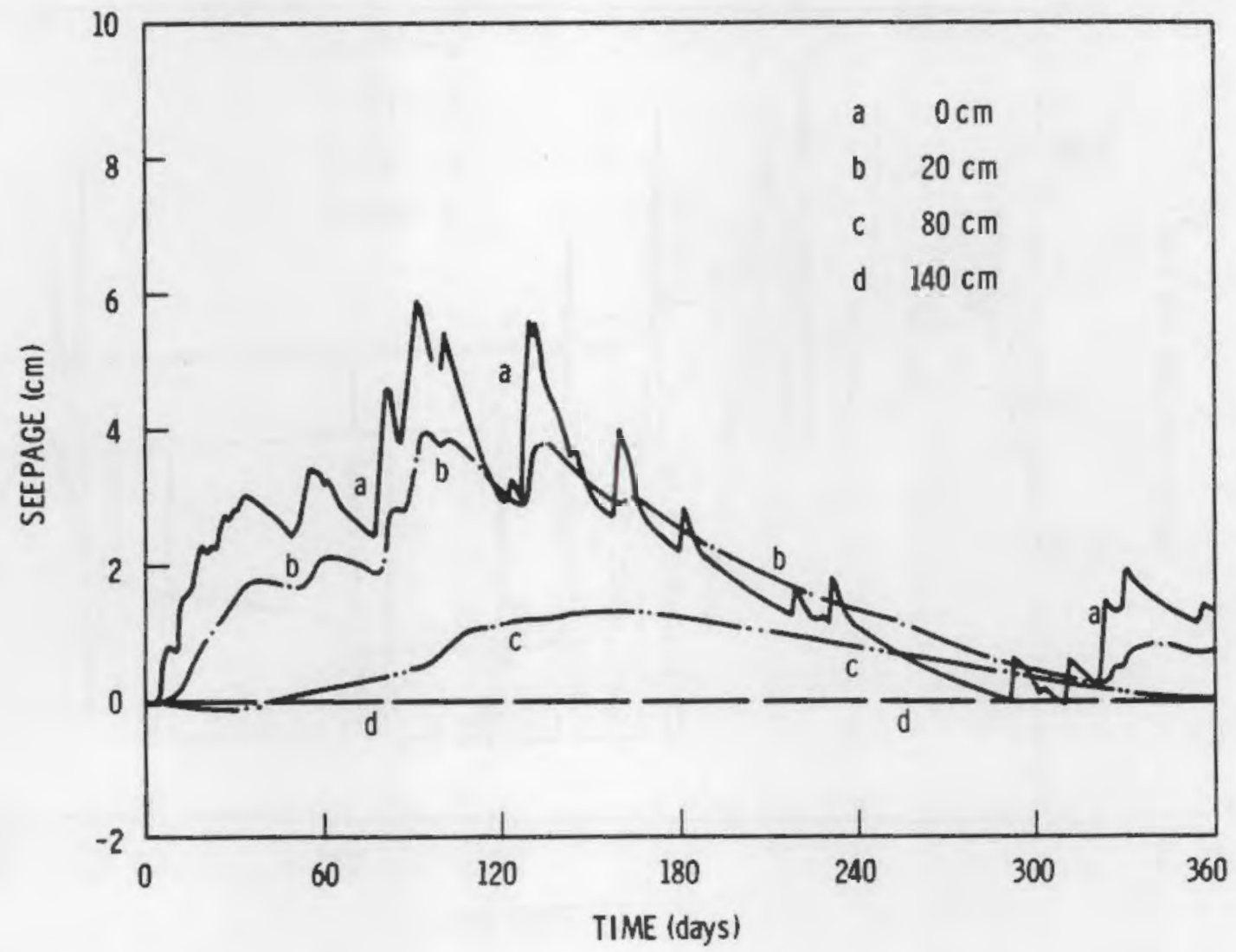

FIGURE 23. Seepage in the Three-Layer Profile, 1979 Climate

\section{Four-Layer Soil Profile}

The objective in designing the profile was to include a clean rock layer above the clay/gravel layer so as to protect this latter layer from water loss due to evaporation and transpiration. A clean rock layer provides a well-known dynamic mechanism for blocking unsaturated water movement. This layer is often referred to as a capiliary barrier. The profile for the same days shown in Figure 27 is shown in Figure 30. The rock layer is seen as the region of very low water content in Figure 30. Greater drying near the surface is evident for the four-layer profile, mainly as a consequence of the reduced water storage depth in the cover soil.

Graphs of water content and seepage at depths considered in Figures 22 and 24 were nearly the same as those obtained for the four-layer profile with plants, and so are not provided. The yearly transpiration level of $11.7 \mathrm{~cm}$ is 


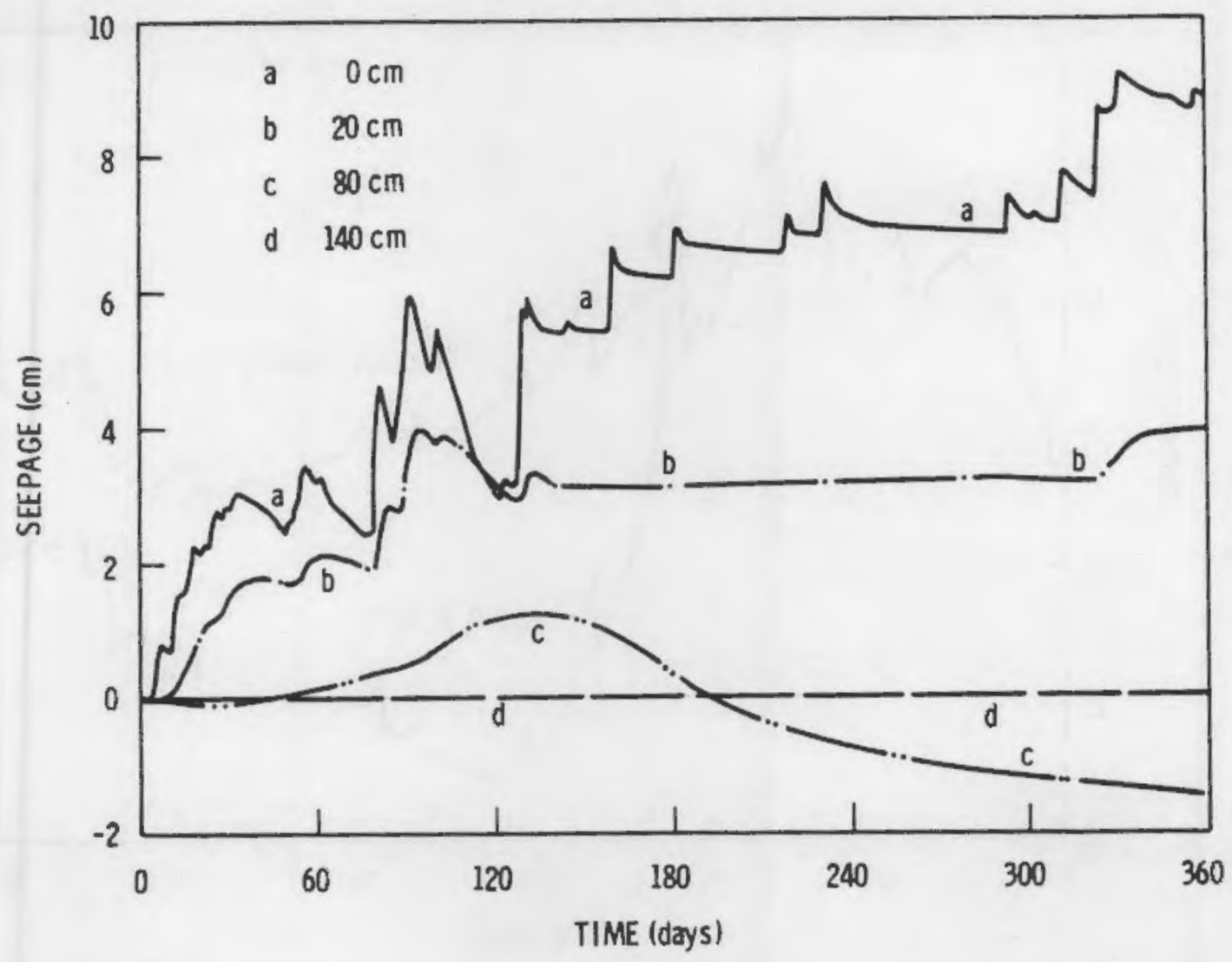

FIGURE 24. Seepage in the Three-Layer Profile with Plants, 1979 Climate

slightly greater than that for the three-layer profile, and evaporation was $13.7 \mathrm{~cm}$. Storage change equaled $-2.7 \mathrm{~cm}$ for a $22.9-\mathrm{cm}$ infiltration without drainage. Thus, water storage decreases about the same amount as in the three-layer case, but water content in the root zone is more reduced in the four-layer profile. Not surprisingly, the transpiration pattern of the fixed growth plant is the same for both profiles (Figure 28). Details of water uptake by the root sink at various depths are shown in Figure 31 . The pattern is very different at deeper depths where rapid drying is not occurring. Delayed arrival at deeper depths of the growing roots is seen.

The overall result is a negligable effect on the water content of the clay/gravel layer. Water content of 0.266 reduces to 0.224 and remains at this value for almost the entire year. The long-term water retention of the clay/gravel layer, however, seems less certain, and continued simulations with variable climatic years were required to ascertain final retention behavior. 


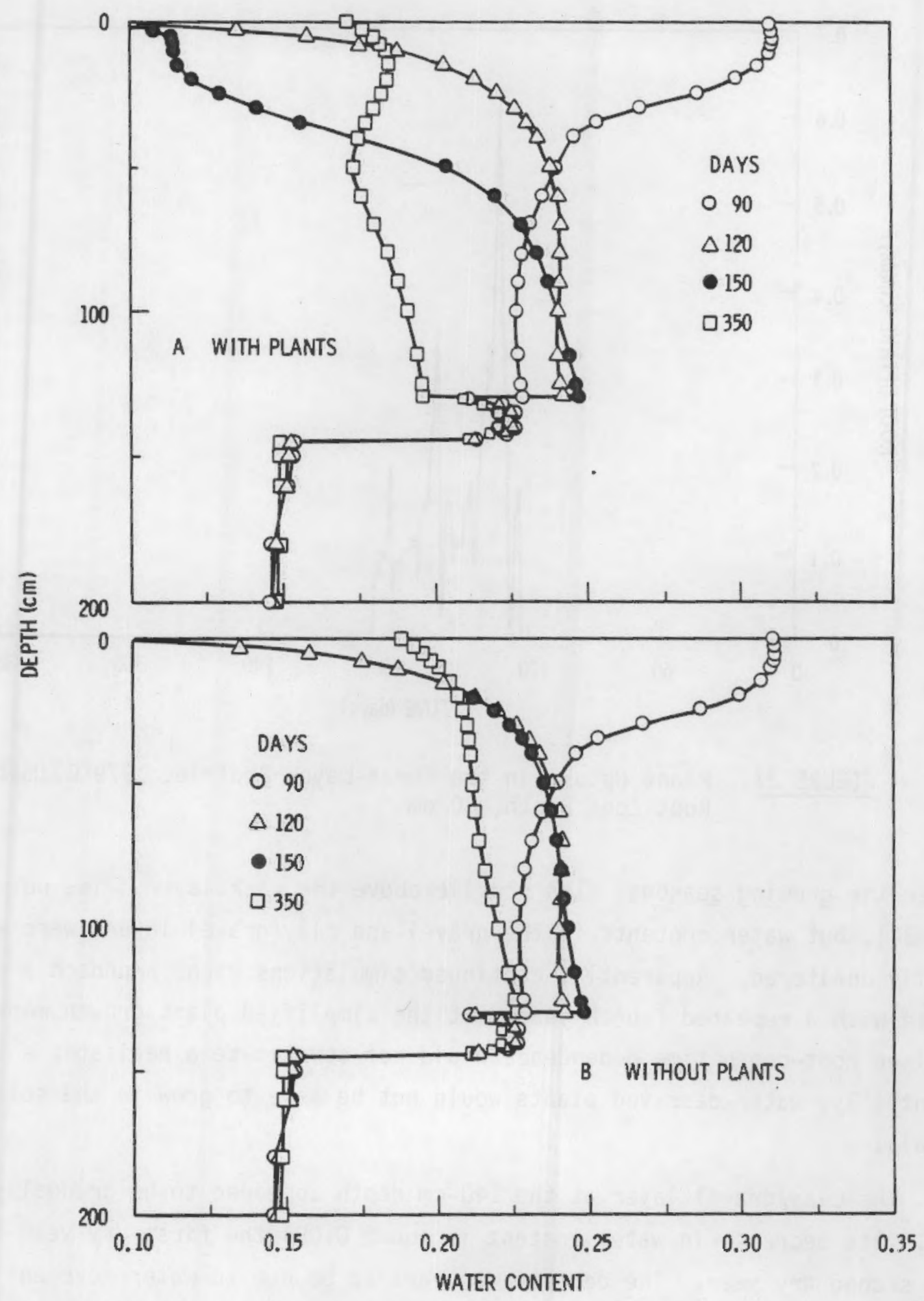

FIGURE 26. Water Content Distributions in the Three-Layer Profile, 1979 Climate, With and Without Plants 


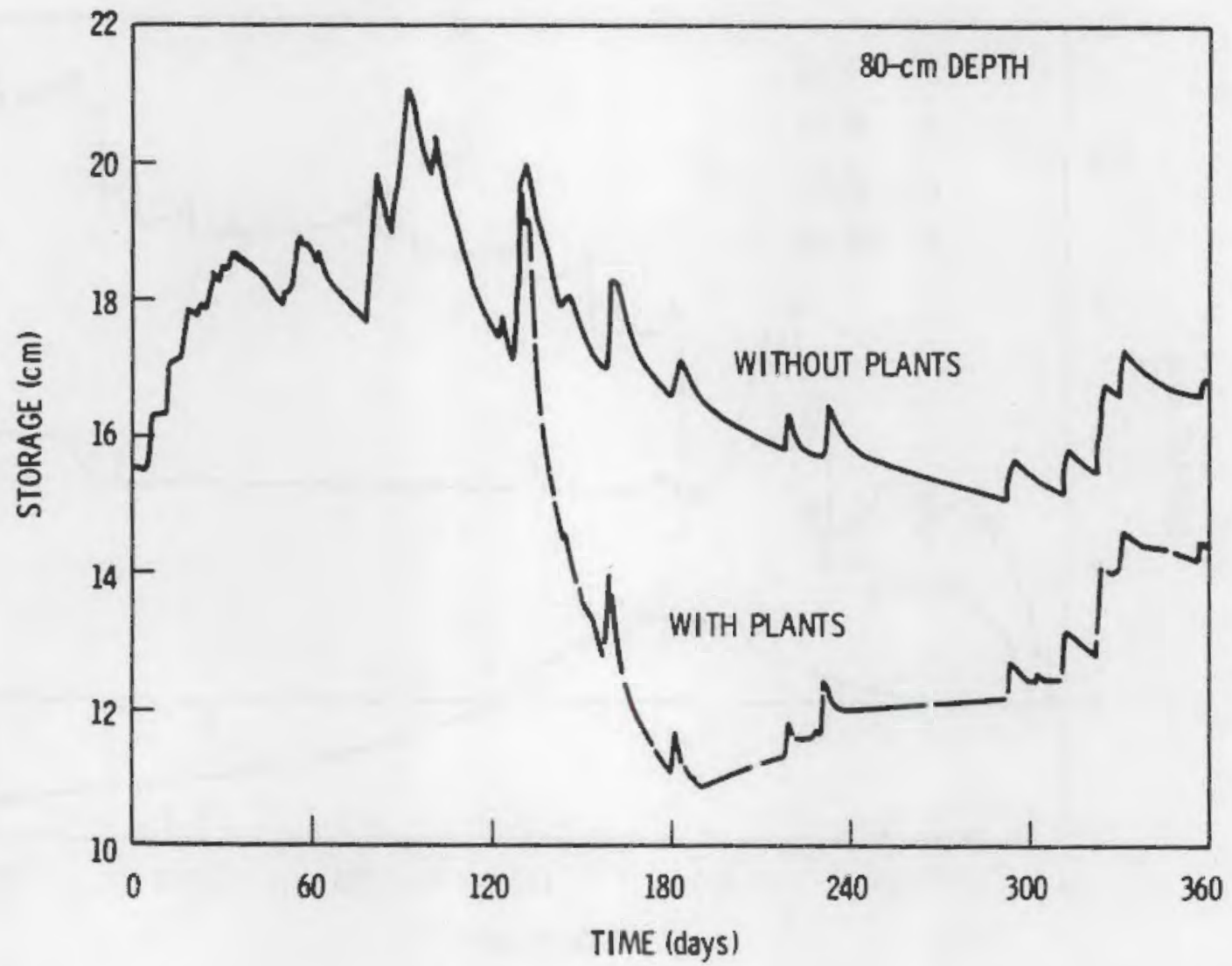

FIGURE 25. Water Storage in the Three-Layer Profile, 1979 Climate, With and Without Plants

Simulation of a Dry Period

To better assess the long-term retention behavior of the clay/gravel layer, a dry period was simulated as a repeated series of two 1976 climatic years. The simulation was performed by using the final conditions of the 1979 simulation as initial conditions. Thus, the period under study consisted of a wet season followed by two successive dry seasons. Table 5 gives the water balance in the four-layer profile for the 3-year period.

Water contents for the two dry years displayed a general decrease near the surface. The pattern of water content variation during the first 1976 climatic year (Figure 32) differs from that for 1979 (Figure 22). Soil water storage to the $80-\mathrm{cm}$ depth is shown in Figure 33. The storage to the $20-\mathrm{cm}$ depth indicated an interesting result of repeating the same pattern during and 


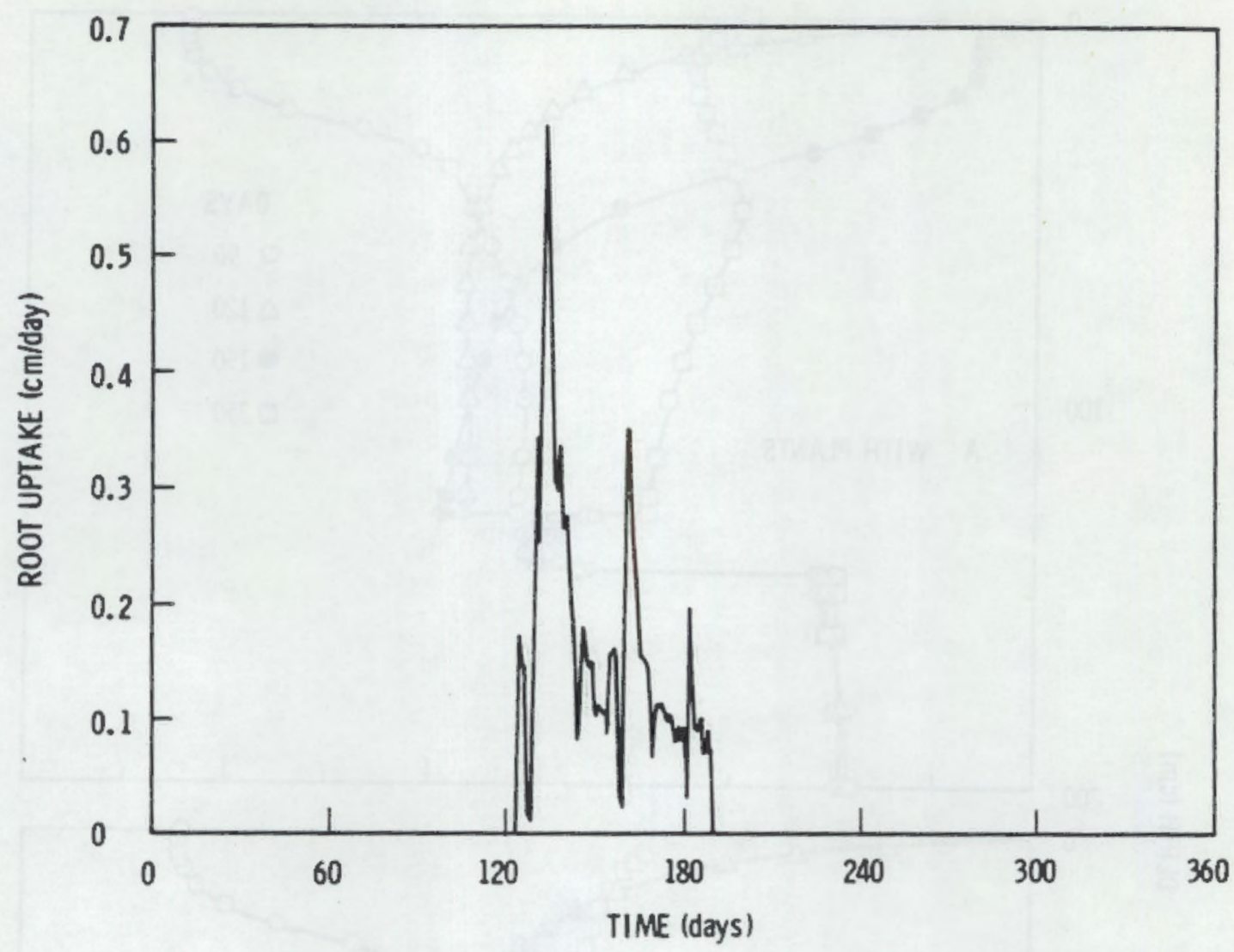

FIGURE 27. Plant Uptake in the Three-Layer Profile, 1979 Climate, Root Zone Depth, $80 \mathrm{~cm}$

after the growing seasons. The profile above the rock layer dried out (Figure 34), but water contents in the gravel and clay/gravel layers were essentially unaltered. Apparently, continued simulations might approach a steady state with a repeated fourth year, but the simplified plant growth model with a fixed root-depth time dependence would not constitute a realistic situation. Eventually, water-deprived plants would not be able to grow in the soil profile at all.

The clay/gravel layer at the 140-cm depth appeared to be gradually drying out. Its decrease in water content is about 0.004 the first dry year and 0.003 the second dry year. The decrease appears to be due to water movement into the tailings. At the rock and clay/gravel layer interface, moisture movement is upward into the gravel, so that drying seems to take place from both sides of the clay/gravel layer. With the water content decreasing at a rate of 0.003 , 


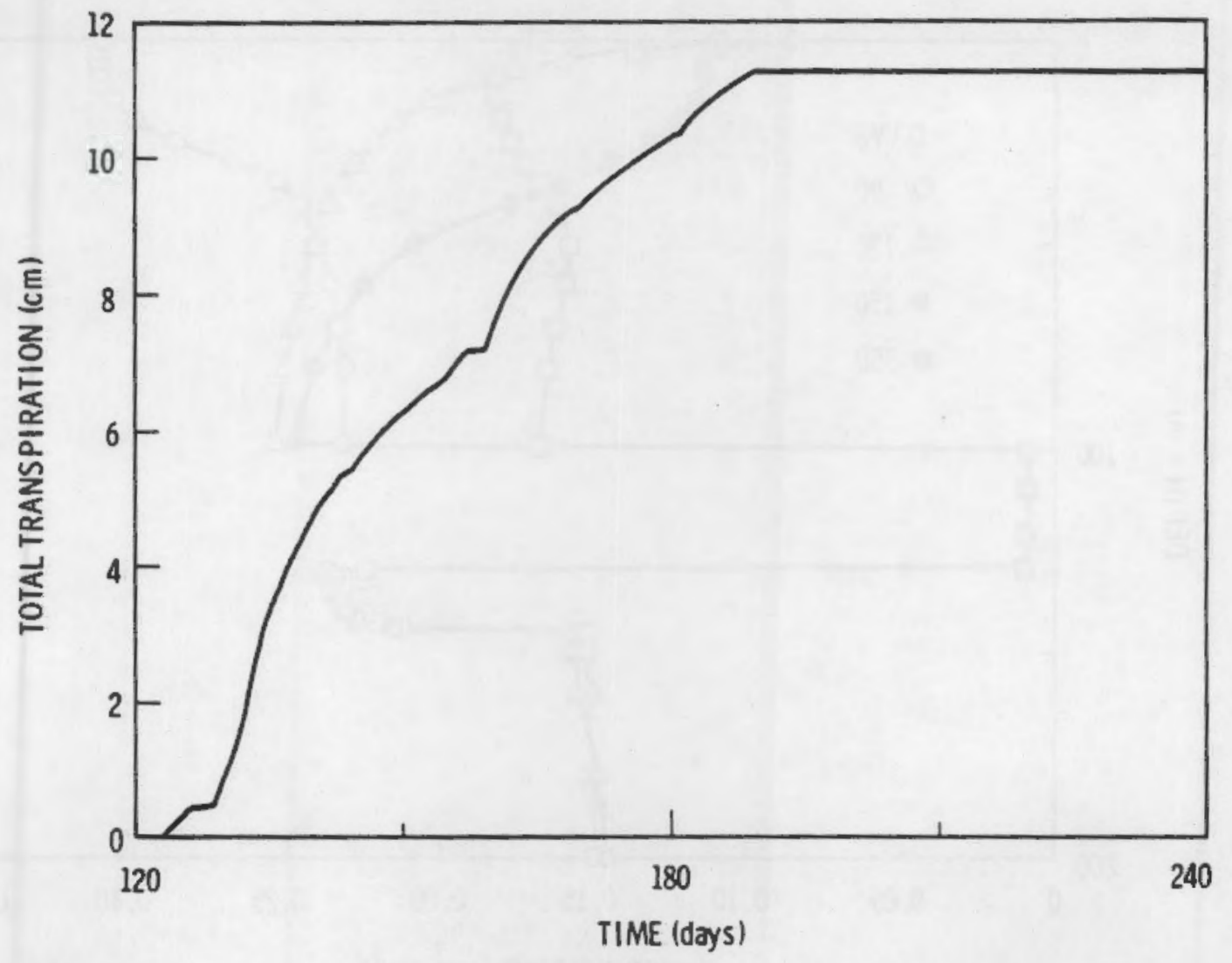

FIGURE 28. Transpiration for Plants in the Three-Layer Profile, 1979 Climate

about 16 years would be required to reduce the water content at the $140-\mathrm{cm}$ depth from 0.217 to 0.170 , the value where vapor flow is estimated to become important and where radon transport increases appreciably. Therefore, about 20 years would be required to dry the clay/gravel layer from initial saturated conditions to vapor flow conditions. The drying time would most likely be longer, however, since the calculation does not include further decreases in the rate of water content with the passage of each year. 


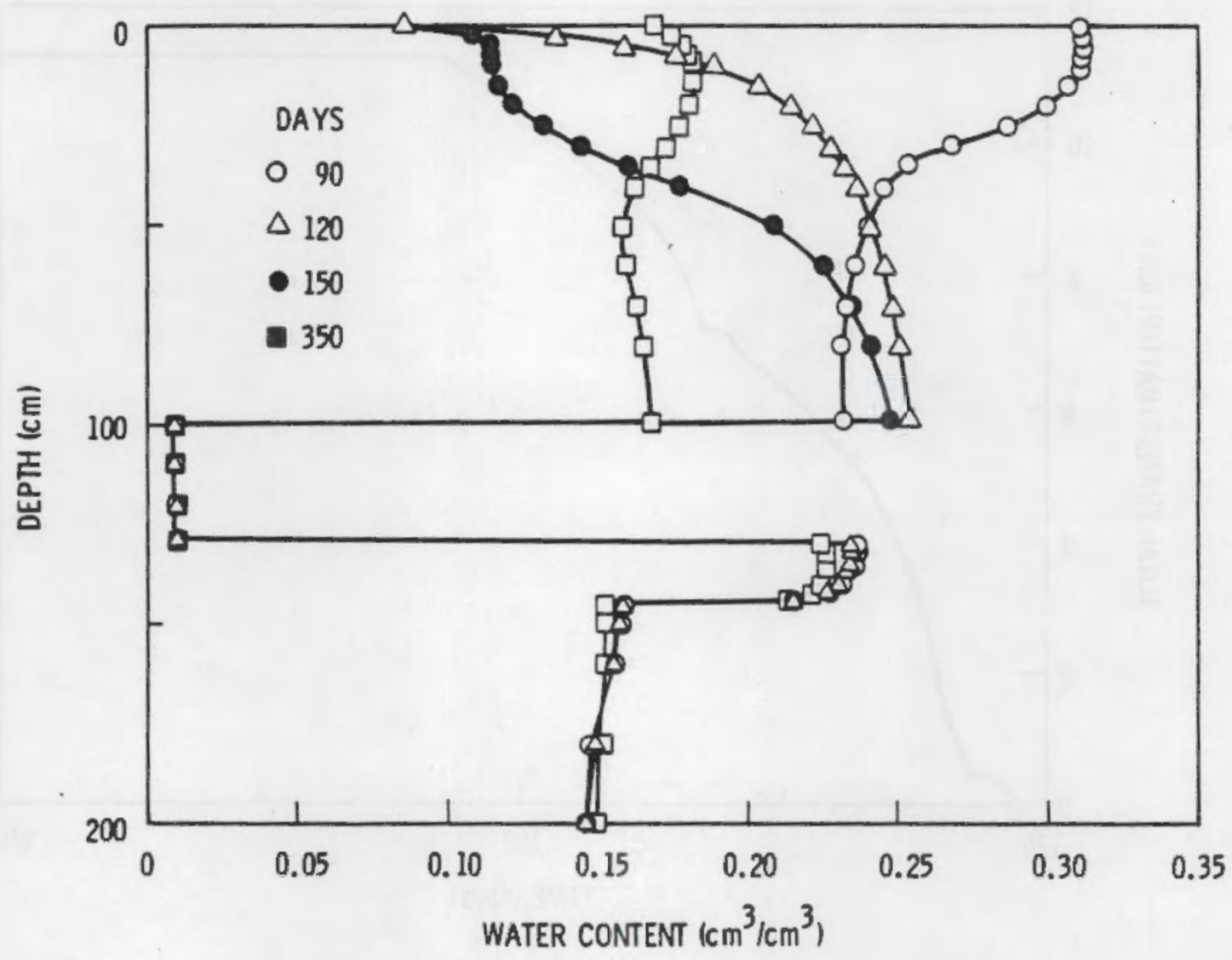

FIGURE 29. Water Content Distributions in the Four-Layer Profile with Plants, 1979 Climate: Days $90,120,150$, and 350

TABLE 4. Water Balance in the Three-Layer Profile for 1979 Climate

\begin{tabular}{|c|c|c|}
\hline Water $(\mathrm{cm})$ & Bare & With Plants \\
\hline PET & 178.3 & 178.3 \\
\hline Evaporation & 21.9 & 13.8 \\
\hline Transpiration & -- & 11.3 \\
\hline Storage Change & 1.4 & -2.4 \\
\hline Infiltration & 23.4 & 22.0 \\
\hline Drainage & 0.0 & 0.0 \\
\hline
\end{tabular}



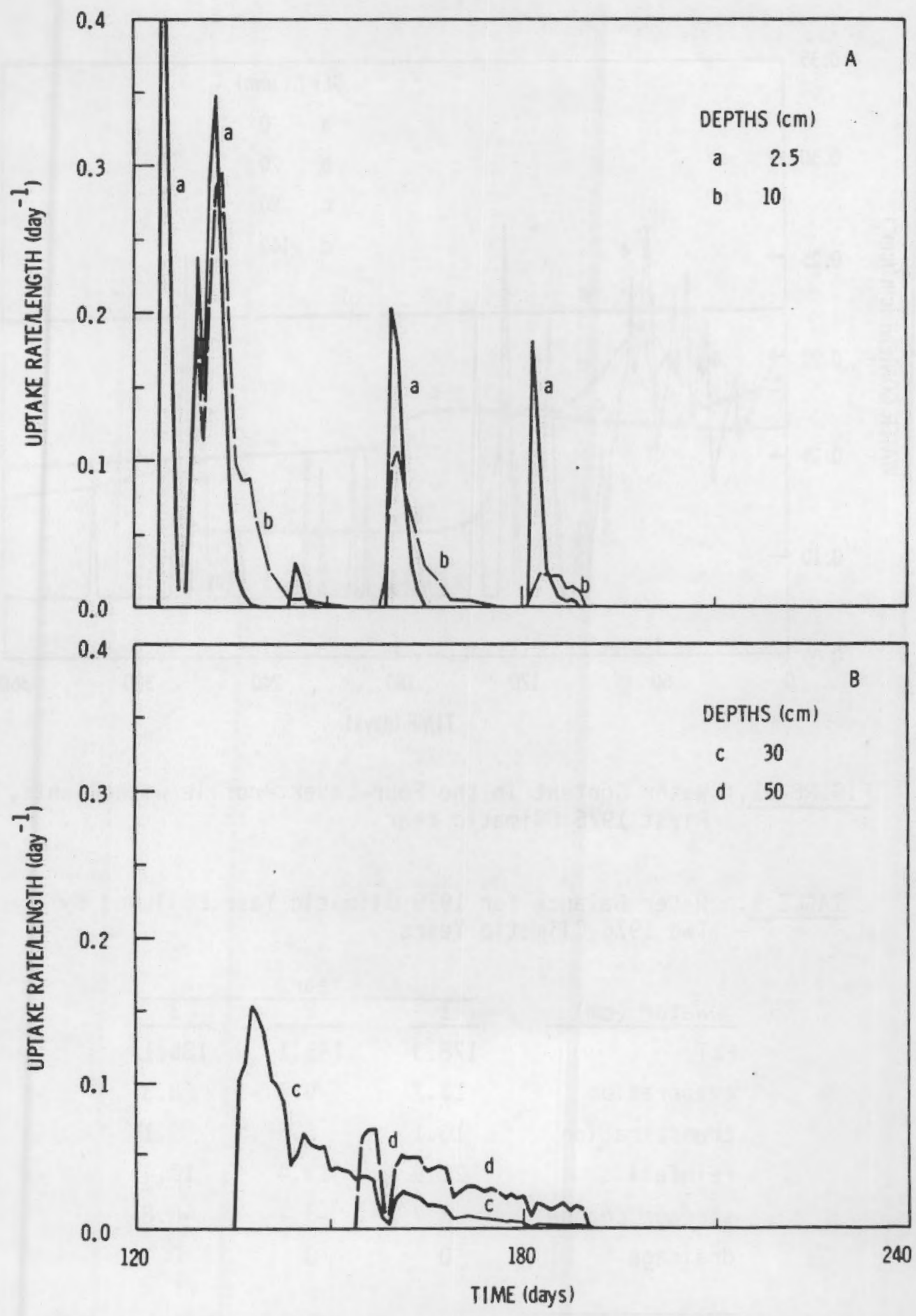

FIGURE 30. Water Uptake Rate Per Unit or Root Length, 1979 Climate: Depths 2.5, 10, 30, and $50 \mathrm{~cm}$ 


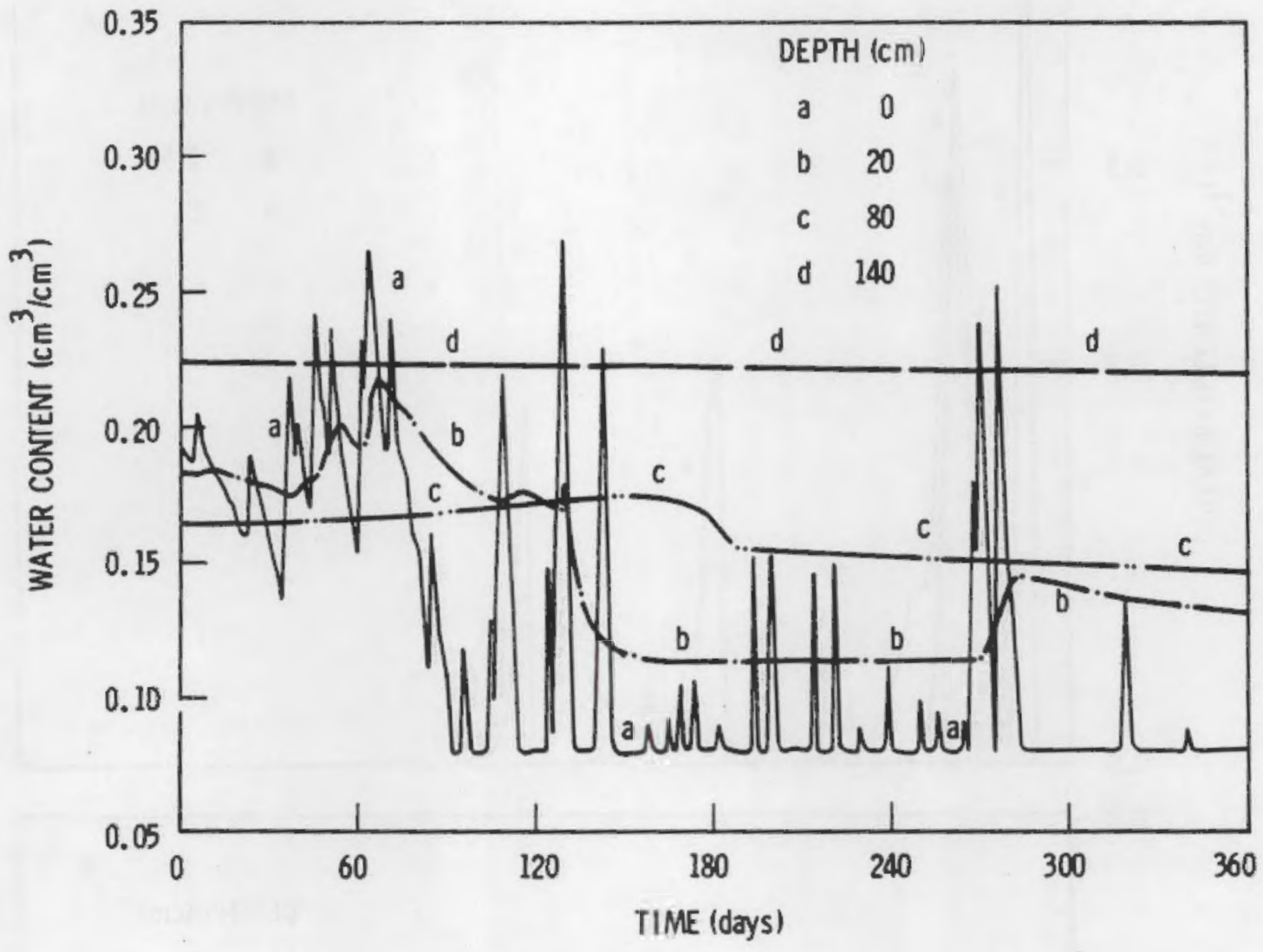

FIGURE 31. Water Content in the Four-Layer Profile with Plants, First 1976 Climatic Year

TABLE 5. Water Balance for 1979 Climatic Year Followed by Two 1976 Climatic Years

\begin{tabular}{|c|c|c|c|}
\hline \multirow[b]{2}{*}{ Water $(\mathrm{cm})$} & \multicolumn{3}{|c|}{ Year } \\
\hline & 1 & 2 & 3 \\
\hline PET & 178.3 & 185.1 & 185.1 \\
\hline evaporation & 13.7 & 9.7 & 8.3 \\
\hline transpiration & 10.1 & 6.5 & 5.1 \\
\hline rainfall & 22.9 & 13.4 & 13.4 \\
\hline storage change & -2.7 & -3.7 & -0.8 \\
\hline drainage & 0 & 0 & 0 \\
\hline
\end{tabular}

storage: $146 . \mathrm{cm}$ initial, $138.8 \mathrm{~cm}$ final 


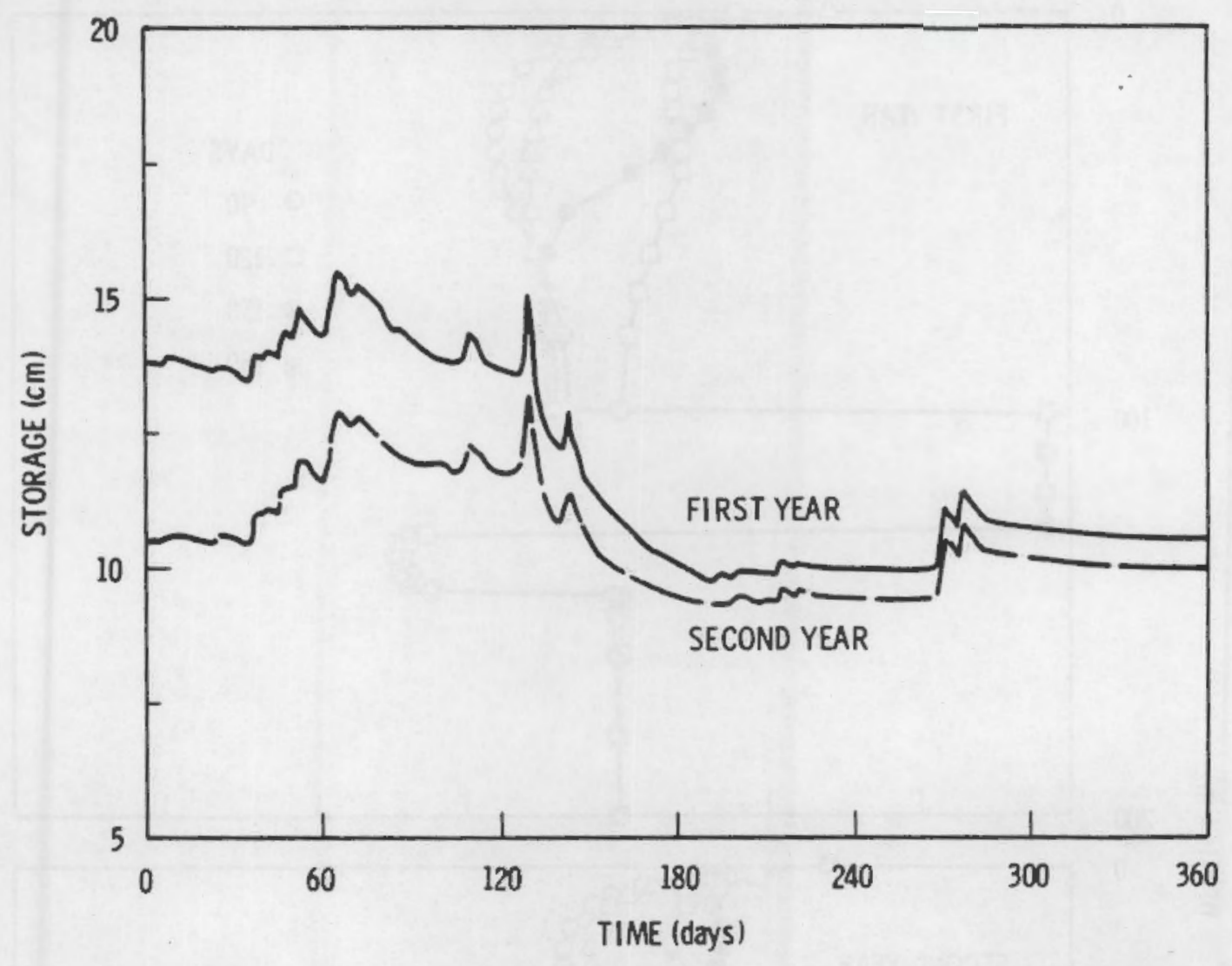

FIGURE 32. Water Storage to the $80 \mathrm{-cm}$ Depth in the Four-Layer Profile, First and Second 1976 Climatic Year with Plants 


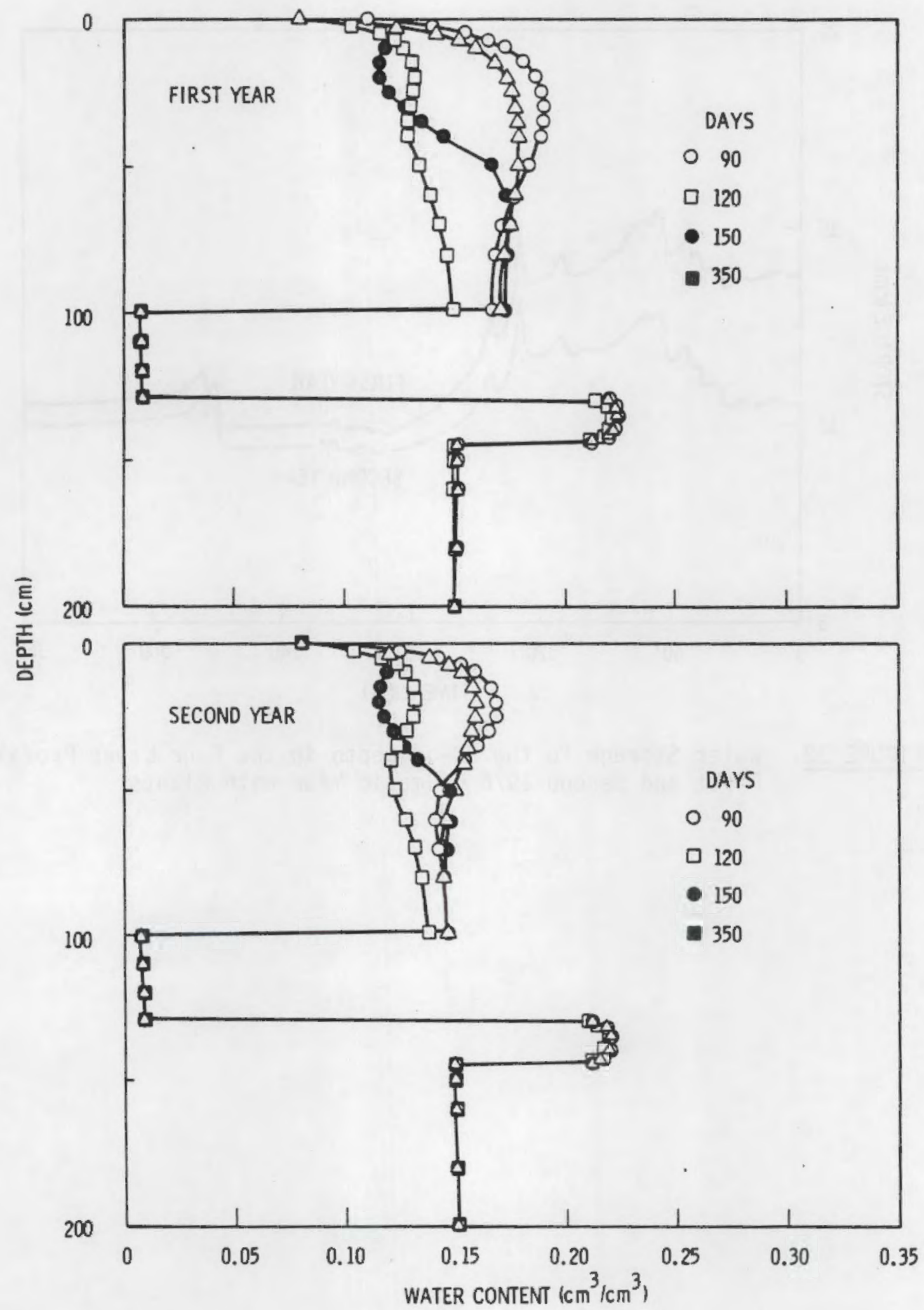

FIGURE 33. Water Content Distributions in the Four-Layer Profile, First and Second Year with Plants; Days 90, 120, 150, and 350 


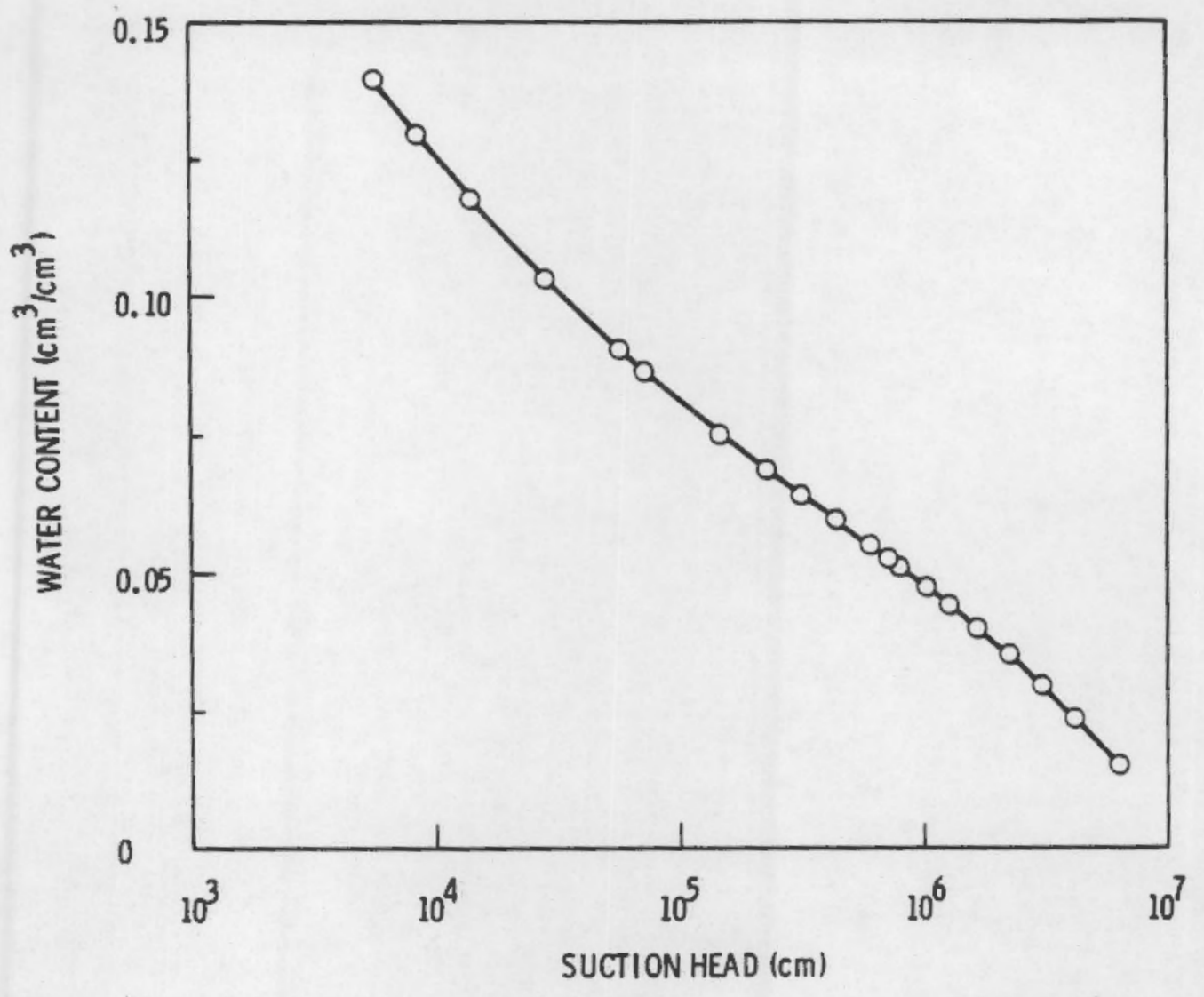

FIGURE 34. Water Vapor Characteristics Curve for Grand Junction Clay 


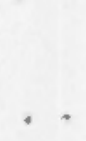




\section{VAPOR FLOW AND DIFFUSION FORMULATION OF SOIL MOISTURE MOVEMENT}

Long-term (1000 yr) assessment of soil-water conditions at an arid region site ultimately requires evaluation of all factors that govern water flow. For these long-term evaluations, the impact of vapor flow mechanisms must be addressed. Under general environmental conditions, the simultaneous transport of both soil moisture (vapor and liquid) and heat must be considered in order to ascertain fuliy the moisture retention behavior of a soil profile. The theory of nonisothermal moisture movement in porous media as developed by Philip and DeVries (1957) provides a general approach. Their theory accounts for liquid and vapor transfer under soil moisture gradients and for heat transfer by conduction under temperature gradients and by latent heat of vapor condensation. To treat the soil surface evaporation process under actual climatic conditions, the complete nonisothermal theory seems to be required. Experimental and theoretical studies (Jackson et al. 1974; Sophocleous 1979), however, indicate that an isothermal approach may be adequate under certain moisture conditions, and especially for conditions deeper in a soil profile where abrupt surface temperature variations are suppressed. In an isothermal approach the equations for moisture and heat transfer can be decoupled, allowing one to make independent calculations of moisture and temperature profile distributions. Decoupling is possible when the transfer of latent heat of condensation by flowing vapor is negligible relative to sensible heat conduction.

The equations for modeling nonisothermal moisture movement have been reviewed by Sophocleous (1979) and Hammel (1979), and elements of a simplified isothermal approach have been described by Jackson (1964). Jackson clarified how diffusion of water vapor was related to liquid water content gradient.

The combined movement of liquid and vapor is expressed by:

$$
q=q_{L}+q_{v}
$$

where the liquid flux $G_{L}$ is given by Darcy's Law [Equation (2)], and the vapor flux $q_{v}$ is given by Fick's law of diffusion (Hanks and Ashcroft 1980): 


$$
q_{v}=-D_{v} \frac{d p}{d z}
$$

where the apparent diffusion coefficient is

$$
D_{v}=\alpha(b-\theta) D_{a}
$$

In Equation (27), $\alpha$ is the product of a pore tortuosity factor (usually equal to 0.66 ) and a mass flow factor (usually equal to 1 ). Other parameters in Equations (26) and (27) are defined in Equation (21). Indeed, Equation (21) is based on the diffusion law [Equation (26)]. The general expressions for moisture flux in terms of water content and soil temperature are obtained by applying the chain rule to derivatives of the soil-water potential, $\psi=\psi_{\mathrm{m}}+$ $\psi_{s}$, and vapor density, $\circ$. The fluxes are

$$
q_{L}=-K_{L}(\theta)\left[\frac{\partial \psi}{\partial \theta} \frac{\partial \theta}{\partial z}+\frac{\partial \psi}{\partial T} \frac{\partial T}{\partial z}+1\right]
$$

and

$$
q_{v}=-D_{v}\left[\frac{\partial \rho}{\partial \theta} \frac{\partial \theta}{\partial z}+\frac{\partial \rho}{\partial T} \frac{\partial T}{\partial z}\right]
$$

By defining isothermal and nonisothermal effective diffusion coefficients for liquid and vapor and applying the continuity equation to the total flux (25), the generai soil moisture flow equation is obtained as

$$
\frac{\partial \theta}{\partial t}=\frac{\partial}{\partial z}\left[D_{\theta} \frac{\partial \theta}{\partial z}+K_{L}(\theta)+D_{T} \frac{\partial T}{\partial z}\right]
$$

where

$$
D_{\theta}=D_{\theta L}+D_{\theta V}
$$

with

$$
D_{\theta L}=K_{L}(\theta) \frac{\partial \psi}{\partial \theta}
$$




$$
D_{\theta v}=D_{v} \frac{\partial \rho}{\partial \theta}=D_{v} \rho_{s} \frac{\partial}{\partial \theta}\left(p / p_{S}\right)
$$

and

$$
D_{T}=D_{T L}+D_{T V}
$$

with

$$
\begin{aligned}
& D_{T L}=D_{V} \frac{\partial \rho}{\partial T}=D_{V}\left(p / p_{S}\right) \frac{\partial \rho_{S}}{\partial T} \\
& D_{T V}=K_{L}(\theta) \frac{\partial \psi}{\partial T}
\end{aligned}
$$

To obtain Equation (30), the moisture per unit volume of soil contained as vapor is neglected since it is small compared with water content. In Equations (32) and (33), the relative humidity is equated with the relative vapor pressure, $p / p_{s}$, by assuming an ideal gas law for water vapor. The isothermal flow equation is obtained as a special case of Equation (30) by assuming $\partial T / \partial Z=0 . D_{e L}$ is called the soil-water diffusivity, and $D_{e v}$ is called the isothermal vapor diffusivity. Under isothermal conditions the movement of moisture in the soil is governed by the sum of those diffusivities. Jackson (1964), Rose (1968), and Scotter (1976) have demonstrated the experimental resolution of diffusivity into its components. Diffusivity is found to decrease with decreasing water content when flow is dominated by liquid flow. As moisture flow becomes dominated by vapor flow, diffusivity shows an increase to a local maximum, which is followed by a continued decrease to zero as water content approaches zero.

\section{WATER VAPOR CONDUCTIVITY}

A considerable simplification of the mathematical description of isothermal moisture flow in a layered profile is achieved by representing vapor flow in terms of a conductivity instead of a diffusivity formulation. Rose (1968) demonstrated from experiment the separation of hydraulic conductivity into liquid capillary and vapor flow components, but an explicit expression for vapor conductivity was not provided. The UNSAT model can be extended into the isothermal vapor flow region as follows. 
The relation between relative humidity and soil water potential [Equation (5)] gives:

$$
\frac{\partial \psi}{\partial \theta}=\frac{R T}{\left(p / p_{s}\right)} \frac{\partial}{\partial \theta}\left(p / p_{s}\right)
$$

By substituting Equation (35) into Equation (32) and defining vapor conductivity $K_{v}$ similar to that for liquid, Equation (31), we get

$$
K_{V}(\theta)=\frac{a D_{a}}{R T} \rho_{s}(b-\theta)\left(p / p_{s}\right)
$$

Total soil moisture conductivity is then

$$
K(\theta)=K_{L}(\theta)+K_{V}(\theta)
$$

Vapor conductivity is made an explicit function of $\theta$ by using the isotherm Equation (6). If an isotherm is not available, Equation (36) can be expressed entirely in terms of the extended soil-water retention characteristic by using

$$
p / p_{s}=e^{\psi / R T}
$$

The advantage of using a conductivity formulation is gained in the assumed continuity of matric potential over a layered soil profile. Total moisture flux, however, is slightiy altered as expressed by

$$
q=-K(\theta)\left[\frac{\partial \psi}{\partial z}+G(\theta)\right]
$$

where

$$
\begin{aligned}
& G(\theta)=X_{L}(\theta) / K(\theta) \\
& G \text { satisfies: } 0 \leq G \leq 1
\end{aligned}
$$


Unlike in the diffusivity formulation of vapor flow, the behavior of Equation (36) is immediately clear. Vapor conductivity has an overall maximum at some intermediate water content since it is composed of the product of strictly decreasing and increasing functions of water content, $(\phi-\theta)$ and $p / p_{S}$, respectively.

Typical values for the physical parameters of Equation (36) for $20^{\circ} \mathrm{C}$ are $\alpha=0.66$

$D_{a}=0.24 \mathrm{~cm}^{2} / \mathrm{sec}=0.864 \times 10^{3} \mathrm{~cm}^{2} / \mathrm{hr}$

$o_{s}=1.73 \times 10^{-5} \mathrm{~g} / \mathrm{cm}^{3}$

$R T=1334.8$ bars $=1.335 \times 10^{6} \mathrm{~cm}$.

The above values yield

$\mathrm{aD}_{\mathrm{a}} \rho_{\mathrm{s}} / \mathrm{RT}=7.4 \times 10^{-9} \mathrm{~cm} / \mathrm{hr}$

and

$$
K_{v}=\left(7.4 \times 10^{-9}\right) 6(1-\theta / 6) p / p_{S}
$$

Thus, $7.4 \times 10^{-9} 6(\mathrm{~cm} / \mathrm{hr})$ is the upper limit for vapor conductivity since $1-\theta / \phi$ and $p / p_{s}$ each have a maximum value of 1 . Rose (1968) indicates similar magnitudes for $K_{v}$. In view of Equation $(40)$, the vapor conductivity makes a negligible contribution until the capillary conductivity reduces to a comparable magnitude of $10^{-8} \mathrm{~cm} / \mathrm{hr}$.

Vapor conductivity was caiculated for the four soils by using Equation (40) with Equation (38) (Figure 2). The retention characteristics used for the simulations, as shown in Figure 1, were employed to estimate relative humidity from Equation (38). In the dry range with pressure heads between $-5000 \mathrm{~cm}$ and $-10^{6}$, the retention characteristics were represented by a linear relation between $\theta$ and $\log (-\psi)$. For pressure heads within the indicated dry range, the log-linear relation provides an adequate approximation of the actual characteristic associated with a measured isotherm described by Equation (6). Figure 35 shows the section of characteristics associated with the isotherm for Grand Junction clay soil shown in Figure 36. Use of a log-linear relation provides a mathematical simplification and a substantial reduction in required isotherm measurements, since then only two points are needed to define the 


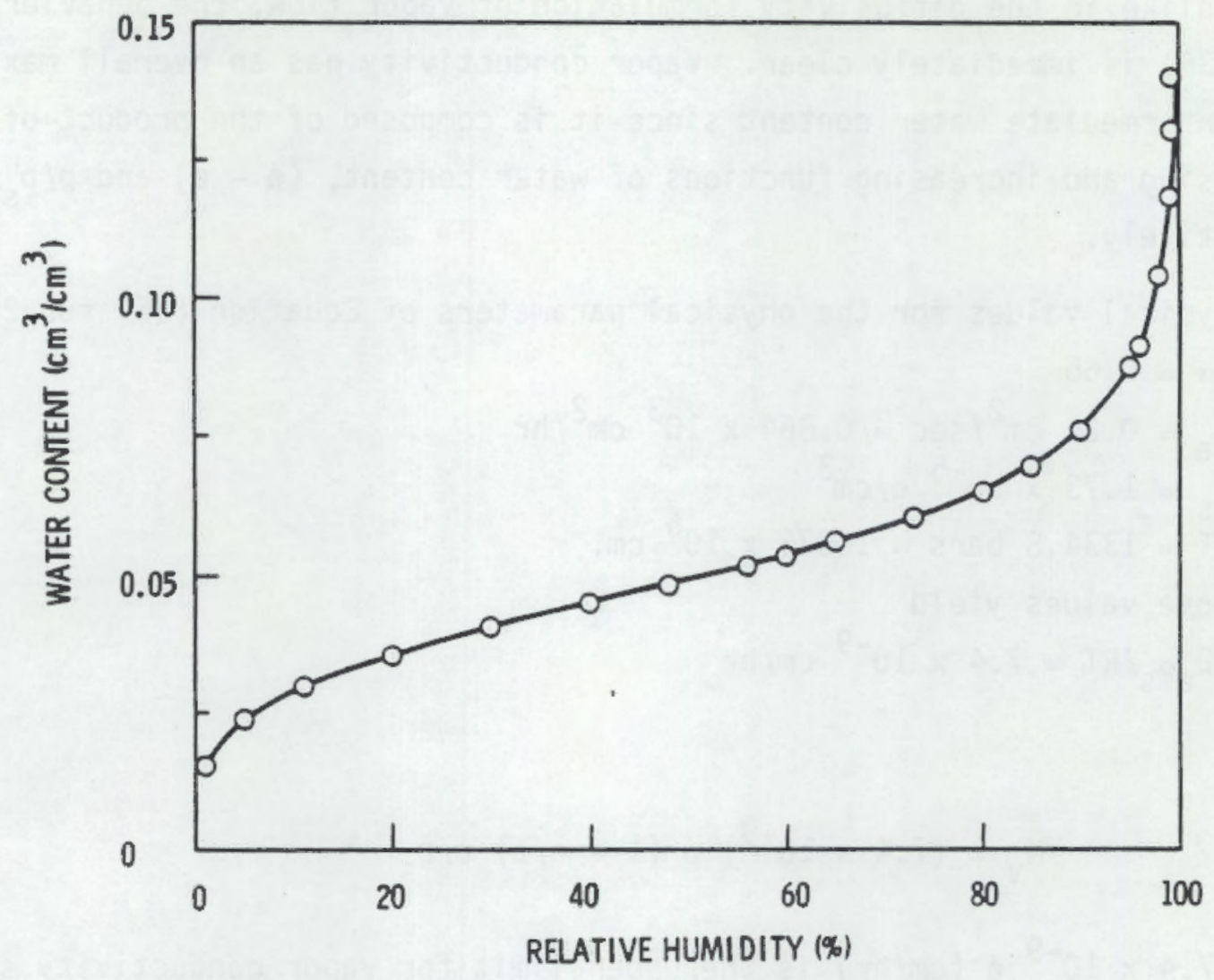

FIGURE 35. Water Vapor Isotherm for Grand Junction Clay

curve. Water content measured at air-dry conditions is often sufficient to establish the lowest point of a characteristic. The error introduced in Equation (36) by using a linear relation between $\theta$ and $\log (-\psi)$ as an approximation appears to be minor, since relative humidity is somewhat insensitive to the specific shape of the charactertistic curve. Figure 37 shows the vapor isotherms passing through end points with different values of parameter $C$ used in Equation (6), which are typical of values for clay soil. Values of $C$ are 1.6 for Cache clay (Fink and Jackson 1971) and 7.5 for bentonite (this study). Values of $A$ and $B$ obtained by passing the curve through the end points at $a$ pressure head of $-5000 \mathrm{~cm}$ and $-10^{6} \mathrm{~cm}$ did not differ greatly. Actual measurements are also shown in Figure 37 , and fall between the calculated isotherms. The corresponding portions of the characteristic curves are shown in Figure 38. A log-linear relation falls between the two curves. Hence, the associated isotherm would fall between the isotherms of Figure 37 . Thus, the 


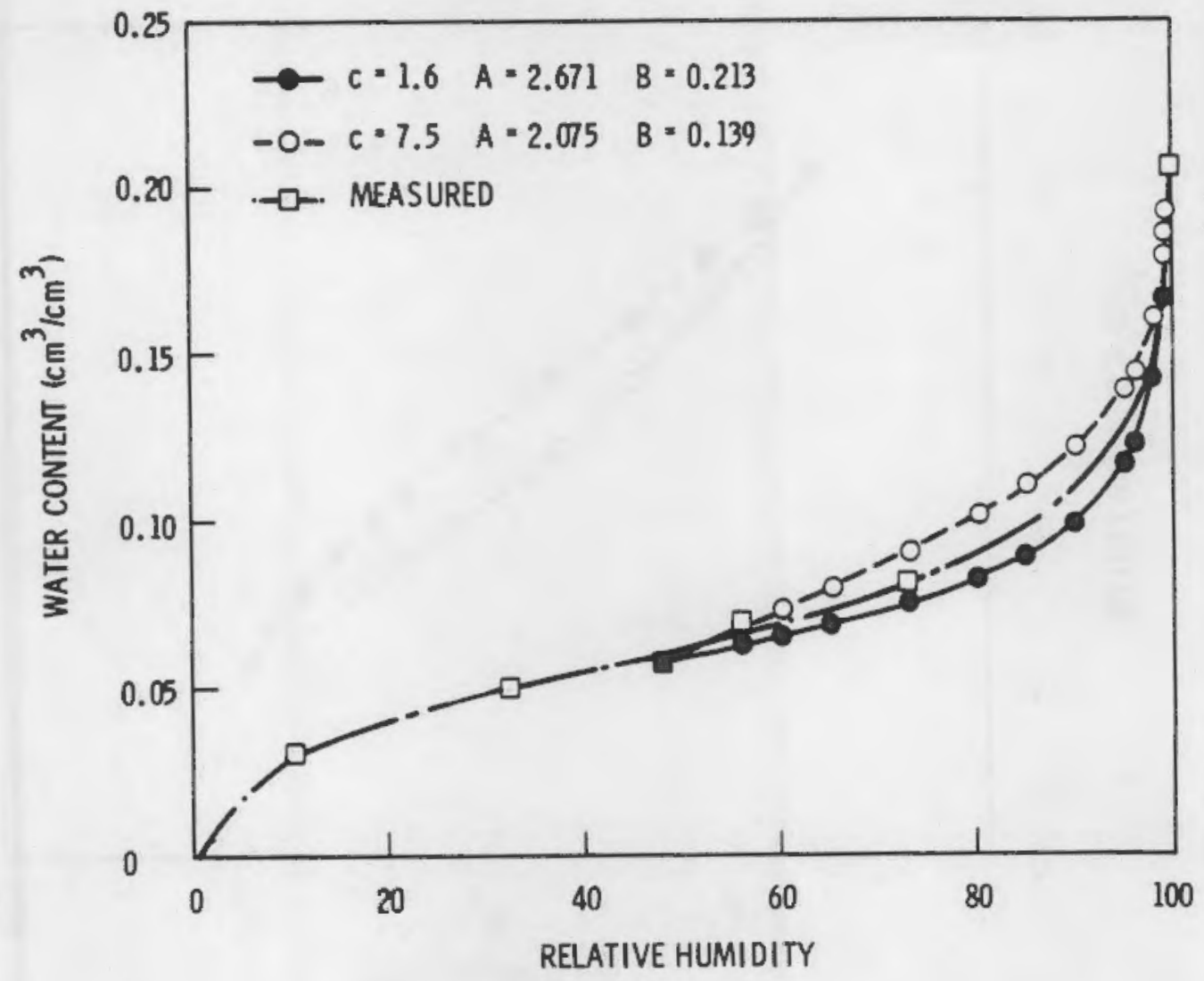

FIGURE 36. Water Vapor Isotherm for Clay/Gravel Mix and Bounding Isotherms Based on $C=1.6$ and $C=7.5$ for Equation (6) (After $F$ ink and Jackson 1973)

error in using a log-linear relation to compute vapor conductivity could not exceed the range of relative humidities between the bounding isotherms for each water content.

Vapor conductivity is displayed for each soil layer along with capillary conductivity in Figures 2. Total isothermal conductivity is the sum of two curves, as in Equation (37). Vapor conductivity was not calculated beyond the shown water content domain for capillary conductivity, but would approach zero as water content decreased to zero, according to Equation (40). Vapor flow is clearly important below the crossing point of the conductivity curves, as water content decreases. Vapor flow would assume major importance for the clayl gravel mix layer at water contents below 0.180. The Klute and Heermann (1978) exponential conductivity function that fits the measurements suggests that 


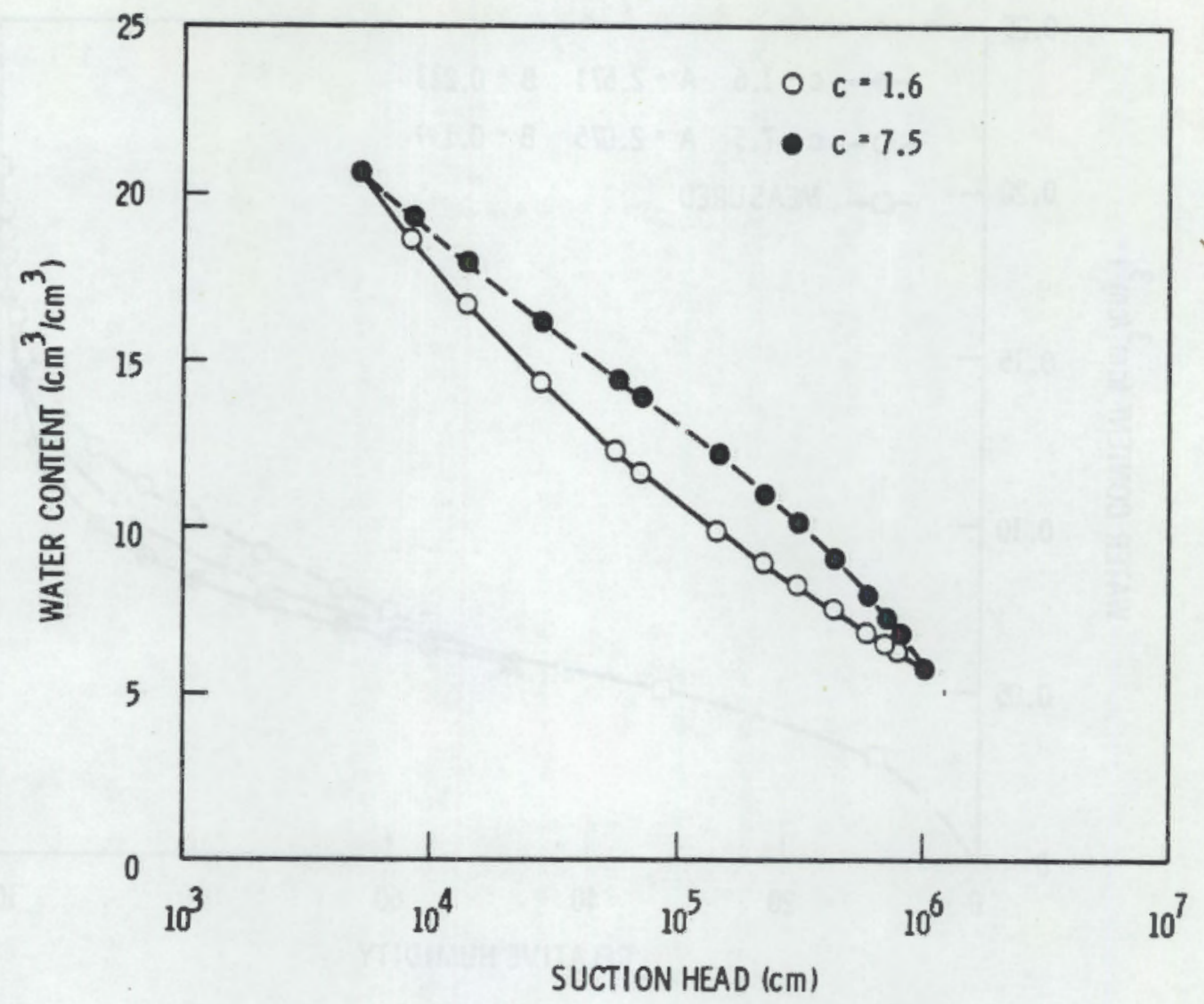

FIGURE 37. Soil-Water Retention Characteristics of Clay/Gravel Mix

vapor conductivity may actually be an order of magnitude greater than predicted by Equation (36), when compared with the calculated liquid conductivity. This result may indicate an enhanced vapor flow mechanism similar to that acting under nonisothermal conditions (Philip and DeVries 1957; Jury and Letey 1979).

Isothermal vapor-phase flow as estimated from Equation (40) would not have been an important factor in the example simulations, except near the soil surface where water content enters the vapor range. That is, water contents below the 20-cm depth for the example simulations never reached values for which vapor conductivity dominates the moisture flow. On the other hand, the temperature gradients ecountered near the soil surface would require the complete nonisothermal moisture flow theory. The present study, however, did not attempt to address nonisothermal flow near the surface; instead, the evapora- 
tion model Equation (21) was employed to represent the overall effect of such flow on deeper regions of the soil profile. A more advanced modeling effort would be necessary to establish the detailed surface evaporation process from its more fundamental physical measurements. 



\section{CONCLUSIONS AND FUTURE RESEARCH NEEDS}

The simulations using UNSATV estimated that the clay-rock layer would not dry to isothermal vapor flow conditions for at least $20 \mathrm{yr}$, provided that the modeled dry climate period continued. However, several factors that were not addressed may alter these predictions.

Mechanistic effects of hysteresis in soil-water properties were not accounted for in the simulation. In view of the considerable spatial variability of hydrauilic property measurements usualiy encountered in the field, hysteresis was considered to have minor importance in establishing moisture retention behavior. Nevertheless, hysteresis is a process that should be studied for its importance to radon gas movement. In a fine-grained soil or clay, water contents for the re-wetting cycle can be much less than those during a preceeding drying period, and a greater air-filled porosity can exist with an associated increased radon flux. The importance of such behavior should be evaiuated. Swelling of soils upon wetting may be another behavior of related importance, especially shrinkage of the clay-rock layer upon continued drying.

The unsaturated flow model was extended into the domain of isothermal vapor flow, but no atempt was made to predict nonisothermal effects. In a hot and dry climate such as at Grand Junction, Colorado, the yearly variation of temperature gradients below the profile's surface might cause moisture vapor flow, which must be taken into account. The studies of Jury (1973) and Cary (1979) would provide a basis on which to evaluate the importance of nonisothermal flow. Certainly, the conduction of heat near the soit surface is a major aspect of evaporation and moisture movement; but given the detail of present physical information of climate and soil heat conductivity, the simplified diffusion model of vapor movement was considered adequate.

The modeling of the plant root sink and associated transpiration estimation is perhaps the weakest theoretical component of the unsaturated mo isture movement. An extensive study of a shortgrass prairie ecosystem was available for a nearby location (Farwell 1971) but, as is typical of many such water balance studies, sufficiently detailed data on plant growth and root soil-water 
extraction patterns were not obtained. Information on the growth behavior of water-deprived grasses is needed, particularly to predict results over extended dry periods.

To conclude, it seems certain that these indicated deficiencies in the unsaturated zone modeling $c$ an be overcome by a plan to collect more extensive data. Although many of the involved physical processes are not as yet fully understood, additional data would allow more refined approximations. Assessment of the annual water balance of an earthen cover system for radon gas control has been demonstrated by the use of UNSAT for simulating unsaturated flow, even when limited data are available. Confidence in cover design will improve as additional data are obtained. It is anticipated that over the next year, data from the field study at Grand Junction will be avajlable and will prove useful in verification and calibration of this modeling effort. 


\section{REFERENCES}

Baver, T. D., W. H. Gardner, and W. R. Gardner. 1972. Soil Physics. Fourth Edition, John Wiley \& Sons, Inc., New York, New York.

Bresler, E., D. Russo and R. D. Miller. 1978. "Rapid Estimate of Unsaturated Hydraulic Conductivity Function." Soil Sci. Soc. Am. J. 42(1):170-172.

Campbeil, G. S. 1974. "A Simple Method for Determining Unsaturated Conductivity from Moisture Retention Data." Soil Sci. 177:311-314.

Cary, 3. W. 1966. "Soil Moisture Transport Due to Thermal Gradients. Practical Aspects." Soil Sci. Soc. Am. Proc. 30:428-433.

Cary, J. W. 1979. "Soil Heat Transducers and Water Vapor Flow." Soif Sci. Soc. Am. J. 43:835-839.

Childs, E. C., and W. Collis George. 1950. "The Permeability of Porous Materials. Roy. Soc. (London) Proc. A 201:392-405.

D'Hollander, E. H. 1979. "Estimation of the Pore Size Distribution from the Moisture Characteristic." Water Resour. Res. 15:107-112.

Doorenbos, J., and W. 0. Pruitt. 1975. "Guidelines for Predicting Crop Water Requirements." Irrigation and Drainage Paper. 24:1-179. First Edition. Food and Agricultural Organization of the United Nations, Rome, Italy.

Farwell, A. G. 1971. The Soil water Regime of a Shortgrass Prairie Ecosystem. Ph.D. Thesis, Colorado State University, Agricuiture and Soil Science Dept., Fort Collins, Colorado.

Feddes, R. A., P. J. Kowalik, and H. Zaradny. 1978. Simulation of Field Water Use and Crop Yield. Centre for Agricultural PubTishing and Documentation, Wagenengin, The Netherlands.

Fink, D. H. and R. D. Jackson. 1973. "An Equation for Describing Water Vapor Adsorption Isotherms of Soils." Soil Sci. 116:256-261.

Gardner, W. R., and D. I. Hillel. 1962. "The Relation of External Evaporative Conditions to the Drying of Soils." J. Geophys. Res, 67:4319-4325.

Gee, G. W., and C. S. Simmons. 1979. Characterization of the Hanford 300 Area Burial Grounds. Task III-Fluid Transport and Modeling. PNL-2921, Pacific Northwest Laboratory, Richiand, Washington.

Gee, G. W. and A. C. Campbell. 1980. Monitoring and Physical Characterization of Unsaturated Zone Transport-Laboratory Analysis. PNL-3304, Pacific Northwest Laboratory, Richland, Washington. 
Gee, G. W., A. C. Campbe]l, P. J. Wierenga and T. L. Jones. 1981. Unsaturated Moisture and Radionuclide Transport: Laboratory Analys is and Modeling. PNL-3616, Pacific Northwest Laboratory, Richland, Washington.

Gupta, S. K., K. Tanji, D. Nielsen, J. Biggar, C. Simmons, and J. MacIntyre. 1978. Field Simulation of Soil-Water Movement with Crop Water Extraction. Water Science and Engineering Paper No. 4013. Dept. of Land, Air, and Water Resour., Univ. of Calif., Davis.

Hame 1, J. E. 1979. Modeling Tillage Effects on Evaporation and Seedzone Water Content During Fallow in Eastern Washington. Ph.0. Thesis, Washington State University, Dept, of Agronomy and Soils, Pullman, washington.

Hanks, R. J., and G. L. Ashcroft. 1980. Applied Soil Physics. SpringerVerlag, New York.

HilleT, D. 1977. Computer Simulation of Soil-water Dynamics: A Compendium of Recent Work. International Development Research Center, Ottawa, Canada.

Hillel, D. I., C. H. M. van Bavel, and H. Talpaz. 1975. "Dynamic Simulation of Water Storage in Fallow Soil as Affected by Mulch of Hydrophobic Aggregates." Soil Sci. Soc. Am. J. 39:826-833.

Hinds, W. T. 1975. "Energy and Carbon Balances in Cheatgrass: An Essay in Autecology." Ecological Monograph No. 45:367-388.

Jackson, R. D. 1964a. "Water Vapor Diffusion in Relativeiy Ory Soil: I. Theoretical Considerations and Sorption Experiments." Soil Sci. Soc. Am. Proc. 28:172-176.

Jackson, R. D. 1964b. "Water Vapor Diffusion in Relatively Dry Soil: II. Desorption Experiments." Soil Sci. Soc. Am. Proc. 28:464-466.

Jackson, R. D. 1964c. "Water Vapor Diffusion in Relatively Dry Soil: III. Steady-State Experiments." Soil Sci. Soc. Am. Proc. 28:466-470.

Jackson, R. D. 1965. "Water Vapor Diffusion in Rèlatively Dry Soil: IV. Temperature and Pressure Effects on Sorption Diffusion Coefficients." Soil Sci. Sco. Am. Proc. 28:466-470.

Jackson, R. D., B. A. Kimbali, R. J. Reginato, and F. S. Nakayama. 1973. "Diurnal Soil-water Evaporation: Time-Depth-Flux-Patterns." Soil Sci. Am. Proc. 37:505-509.

Jackson, R. D., R. J. Reginato, B. A. Kimball, and F. S. Nakayama, 1974. "Diurnai Soil-water Evaporation: Comparison of Measured and Calculates Soij-Water Fluxes." Soil Sci. Soc. Amer. Proc. 38:861-866.

Jackson, R. D., S. B. Idso, and R. J. Reginato. 1976. "Calculation of Evaporation Rates During Transition from Energy-L imiting to Soi1-Limiting Phases Using Albedo Data." Water Resour. Res. 12:23-26. 
Jury, w. A. 1973. Simultaneous Transport of Heat and Moisture Through a Medium Sand. Ph.o. Dissertation, Univ. Microfilms, Ann Arbor, Michigan, $191 \mathrm{pp}$.

Jury, W. A. and J. Letey. 1979. "Water Vapor Movement in Soil: Reconciliation of Theory and Experiment." Soil Sci. Soc. Am. J. 43:823-827.

Jury, W. A., and E. E. Miller. 1974. "Measurement of the Transport Coefficients for Coupled Flow of Heat and Moisture in a Medium Sand. "Soi? Sci. Soc. Am. Proc. 38:551-557.

Klute, A, 1972. "The Determination of the Hydraulic Conductivity and Diffusivity of Unsaturated Soils." Soil Sci. 113:264-276.

Klute, A., and 0. F. Heermann. 1978. "Water Movement in Uranium Mill Tailings Profiles." Technical Note ORP/LV-78-8, Office of Radiation Programs Las Vegas Facility, U.S. Environmental Protection Agency, Las Vegas, Nevada.

Kunze, R. J., G. Uehara and K. Graham. 1969. "Factors Important in the Calculation of Hydraulic Conductivity." Soil Sei Soc. Am. Proc. 32:760-765.

Marshal1, T. J., and 3. W. Holmes. 1979. Soil Physics. Cambridge Univ. Press, Cambridge, London.

Mayer. D. W., R. W. Nelson and G. W. Gee. Radon Diffusion Through Multilayer Earth Covers: Models and Simulation. PNL-3989, Pacific Northwest Laboratory, Richland, Washington.

Millington, R. J., and J. P. Quirk. 1961. "Transport in Porous Media." Trans. Faraday Soc. 57:1200-207.

Molz, F. J., J. M. Davidson, and E. W. Tollner. 1979. Unsaturated-Zone Water. Papers in Hydrology, Seventeenth General Assembly, International Union of Geodesy and Geophysics, Canberra, Australia. Aner. Geophysical Union $17: 1221-1239$.

Mualem, Y. 1976. "A New Model for Predicting the Hydraulic Conductivity of Unsaturated Porous Media." Water Resour. Res. 12:513-522.

Nielsen, D. R., J. W. Biggar and K. T. Erh. 1973. "Spatial Variability of Field-Measured Soil-Water Properties." Hilgardia 42:215-260.

Nunn, J. R., L. J. Bledsoe, and R. D. Burman. 1970. Models for Inferring Evaporation from Meteorological Measurements. Tech. Report 47, Grasslands Bioine, U.S. International Biological Program.

Philip, J.R. 1957. "The Theory of Infiltration: I." Soil Sci. 83:345-357. 
Philip, J. R. 1957. Physics of Water Movement in Porous Solids. Special Report 409, Highway Research Board, National Academy of Sciences, National Research Council, Pub. 629.

Philip, J. R., and D. A. DeVries. 1957. "Moisture Movement in Porous Materials Under Temperature Gradients." Trans. Am. Geophys. Union $38: 222-232$.

Reisenauer, A. E. 1972. Calculations of Soil Hydraulic Conductivity from Soil-water Retention Relationships. BNWL-1710, Pacific Northwest Laboratory, Richland, Washington.

Reisenauer, A. E., D. B. Cearlock, and C. A. Bryan. 1975. PartiallySaturated Transjent Groundwater Flow Model Theory and Numerical Implementation. BNWL-1713, Pacific Northwest Laboratory, Richland, Washington.

Rose, D. A. 1968. "Water Movement in Porous Materiais. III. Evaporation of Water from Soil." Brit. J. Appl. Phys. (J Phys. D), Ser. 2, 1:1779-1791.

Scotter, D. R. 1976. "Liquid and Vapor Phase Transport in Soil." Aust. J. Soil Res. 14:33-41.

Simmons, C. S., D. R. Nielsen, and J. W. Biggar. 1979. "Scaling of FieldMeasured Soil-Water Properties I. Methodology II. Hydraulic Conductivity and Flux." Hilgardia 47:77-173.

Sophocleous, M. 1979. Analys is of Water and Heat Flow in UnsaturatedSaturated Porous Media, Water Resour. Res., 15:1195-1206.

Taylor, S. A., and J. W. Cary. 1964. "Linear Equations for the Simultaneous Flow of Matter and Energy in a Continuous Soil System." Soil Sei. Soc. Am. Proc. 28:167-172.

Van Genuchten, M. Th. 1978. Calculating the Unsaturated Conductivity With a New Closed-Form Analytical Model. 78-WR-08 p.65. Department of Civil Engineering, Princeton, New Jersey.

White, I., D. E. Smiles, and K. M. Perroux. 1979. "Absorption of Water by Soil: The Constant Flux Boundary Condition." Soil Sci. Soc. Am. J. 43:659-664. 
APPENDIX A

MOISTURE FLOW EQUATION IN FINITE OIFFERENCE FORM 


\section{APPENDIX A}

\section{MDISTURE FLOW EQUATION IN FINITE DIFFERENCE FDRM}

In the UNSATV program, the Richard's flow Equation (3) with sink term is represented by a Crank-Nicolson implicit finite difference scheme. The total hydrautic head is defined as

$$
H=h+G Z
$$

where $G$ is a gravitational flow angle factor. The finite difference equation is

$$
c_{i}^{j-1 / 2}\left(\frac{h_{i}^{j}-h_{i}^{j-1}}{\Delta t_{j}}\right)=\frac{2}{z_{i+1}-z_{i-1}}\left(q_{j+1 / 2}^{j-1 / 2}-q_{i-1 / 2}^{j-1 / 2}+s_{i}^{j-1 / 2}\right)
$$

where

$$
\begin{aligned}
& c_{i}^{j-1 / 2}=\frac{c_{i}^{j}+c_{i}^{j-1}}{2} \\
& s_{i}^{j-1 / 2}=\frac{s_{i}^{j}+s_{i}^{j-1}}{2} \\
& q_{j}^{j-1 / 2}=\frac{q_{i}^{j}+q_{i}^{j-1}}{2}
\end{aligned}
$$

with

$$
\begin{aligned}
& q_{j+1 / 2}^{j}=k_{i+1 / 2}^{j}\left(\frac{h_{j+1}^{j}-h_{j}^{j}}{\Delta Z_{j+1}}+G\right) \\
& q_{i-1 / 2}^{j}=k_{i-1 / 2}^{j}\left(\frac{h_{i}^{j}-h_{i-1}^{j}}{\Delta Z_{i}}+G\right)
\end{aligned}
$$


and with increments

$$
\Delta z_{i}=z_{j}-z_{i-1}\left(z_{0}=0\right) \text { and } \Delta t_{j}=t_{j}-t_{j-1}\left(t_{0}=0\right)
$$

$i$ denotes the node of depth, $z_{i}$, and $j$ denotes the $j$ 'th time step, $t_{j}$, with increment $\Delta t_{j}$. Hydraulic conductivity between nodes is calculated as ejther

$$
k_{i+1 / 2}^{j}=\frac{k_{i}^{j}+k_{i+1}^{j}}{2} \quad \text { (arithmetic mean) }
$$

or

$$
k_{i+1 / 2}^{j}=\left(k_{i}^{j}\right)^{l-w}\left(k_{i+1}^{j}\right)^{w} \quad \text { (geometric mean) }
$$

where $0 \leq w \leq 1$ and $k_{j}^{j}=k\left(h_{j}^{j}\right)$. The weighted mean conductivity (A.4) is a program option useful when the conductivity changes greatly between nodes, as during movement of a wetting front. Without weighting, internodal fluxes (A.2) are determined by the largest conductivity value.

Equation (A.1) is a non-linear equation to be solved for $h_{i}^{j}(i=1$, ..., N) at each time step $j$, based on the head values from the previous time step $j-1$. To begin the solution, the initial head values and boundary condition must be specified. The difference equation for (A.1) at interior nodes $(i=2, \ldots, N-1)$ is

$$
A_{i}^{\star} h_{i-1}^{\star}+B_{i}^{\star} h_{j}^{j}+C_{j}^{\star} h_{j+1}^{j}=D_{i}^{\star}
$$

where

$$
\begin{aligned}
& A_{i}^{\star}=-k_{i-1 / 2}^{j} / \Delta Z^{\star} \Delta Z_{i} \\
& B_{i}^{\star}=C_{j}^{j-1 / 2} / \Delta t_{j}+\left(k_{j+1 / 2}^{j} / \Delta Z_{j+1}+k_{j-1 / 2}^{j} / \Delta Z_{j}\right) / \Delta Z^{\star} \\
& C_{i}^{\star}=-k_{j+1 / 2}^{j} / \Delta Z^{\star} \Delta Z_{j+1}
\end{aligned}
$$




$$
\begin{aligned}
0_{i}^{*} & =c_{i}^{j-1 / 2} h_{i}^{j-1 / \Delta t_{j}}+\frac{1}{\Delta z^{\star}}\left[k_{i+1 / 2}^{j-1}\left(\frac{h_{j+1}^{j-1}-h_{i}^{j-1}}{\Delta z_{i+1}}+G\right)\right. \\
& \left.-k_{i-1 / 2}^{j-1}\left(\frac{h_{i}^{j-1}-h_{i-1}^{j-1}}{\Delta z_{i}}+G\right)\right]+\left(k_{i+1 / 2}^{j}-k_{i-1 / 2}^{j}\right) G / \Delta Z^{\star}
\end{aligned}
$$

with $\Delta z^{*}=z_{1+1}-z_{i-1}$ for $(i=2, \ldots, N-1)$. The tri-diagona system of Equations (A.5) is solved subject to various boundary condition options listed below.

The fixed surface-head boundary condition is

$$
B_{2}^{*} h_{2}^{j}+C_{2}^{*} h_{3}^{j}=D_{2}^{*}-A_{2}^{*} h_{1}^{j}
$$

for a given $h_{1}^{j}$, [and the system $(A .5)$ begins with $i=2$ ].

The surface flux boundary condition is

$$
B_{1}^{*} h_{1}^{j}+C_{1}^{*} h_{2}^{j}=D_{1}^{*}
$$

where

$$
\begin{aligned}
& A_{1}^{*}=0 \\
& B_{1}^{\star}=c_{1}^{j-1 / 2} / \Delta t_{j}+k_{3 / 2}^{j} / \Delta Z^{\star} \Delta Z_{2} \\
& c_{1}^{*}=-k_{3 / 2}^{j} / \Delta z^{*} \Delta z_{2} \\
& o_{1}^{*}=c_{1}^{j-1 / 2} h_{1}^{j-1} / \Delta t_{j}+\frac{1}{\Delta z^{\star}}\left[k_{3 / 2}^{j-1}\left(\frac{h_{2}^{j-1}-h_{1}^{j-1}}{\Delta Z_{2}}+G\right)\right. \\
& \left.+G K_{3 / 2}^{j}-2 a_{1 / 2}^{j-1 / 2}\right]
\end{aligned}
$$

A. 3 
with $\Delta z^{*}=z_{2}-z_{1}$. The time averaged surface flux $q_{1 / 2}^{j-1 / 2}$ is specified, and the system ( $A .5$ ) begins with $i=1$.

The fixed head lower boundary condition is

$$
A_{N-1}^{*} n_{N-2}^{j}+B_{N-1}^{\star} n_{N-1}^{j}=D_{N-1}^{*}-C_{N-1}^{\star} n_{N}^{j}
$$

where $h_{N}^{j}$ is specified, and the system $(A .5)$ ends with $i=N-1$.

The unit gradient lower boundary condition is

$$
A_{N}^{*} h_{N-1}^{j}+B_{N}^{*} h_{N}^{j}=D_{N}^{*}
$$

where

$$
\begin{aligned}
& A_{N}^{\star}=-K_{N-1}^{j} / \Delta Z^{\star} \Delta Z_{N} \\
& B_{N}^{\star}=C_{N}^{j-1 / 2} / \Delta t j+k_{N-1 / 2}^{j} / \Delta Z^{\star}{ }_{\Delta} Z_{N} \\
& C_{N}^{\star}=0 \\
& D_{N}^{\star}=C_{N}^{j-1 / 2} n_{N}^{j-1} / \Delta t_{j}+\frac{1}{\Delta Z^{\star}}\left[2 q_{N+1 / 2}^{j-1 / 2}\right. \\
& \left.-x_{N-1 / 2}^{j-1 / 2}\left(\frac{h_{N}^{j-1}-h_{N-1}^{j-1}}{\Delta Z_{N}}+G\right)-G K_{N-1 / 2}^{j}\right]
\end{aligned}
$$

with $\Delta z^{*}=Z_{N}-z_{N-1}$. The unit gradient drainage flux is

$$
q_{N+1 / 2}^{j-1 / 2}=\frac{1}{2}\left(K_{N}^{j-1}+k_{N}^{j}\right)
$$

A dynamic water table is defined by a specified fiux for $q_{\mathrm{N}+1 / 2}^{j}$. 
An iterative procedure is used to solve the non-linear system of Equation (A.5). The coefficients $A_{i}^{\star}, B_{j}^{*}, C_{i}^{*}$, and $D_{i}^{\star}$ are evaluated for the $h_{j}^{j-1}$ of the previous time step, and $(A .5)$ is solved for the $h_{j}^{j}$. These values are then used to reevaluate the coefficients and solve for a corrected set of head values. The accuracy of the interative solution is controlled by reducing the time step $\Delta t_{j}$ so that the water mass balance is held within specified error limits.

A special tri-diagonal sequential solution routine is employed to solve (A.5) subject to the given boundary conditions at the profile surface and base. The program proceeds with a surface flux boundary condition whenever evaporation or rainfall is taking place and uses a fixed surface head condition when ponding occurs. If the surface head falls outside specified wet or dry limits during application of surface flux conditions, the program automatically switches to a fixed surface head condition so that a limiting maximum infiltration rate or evaporation rate is imposed by the soil surface.

Soil-Water Mass Balance

Soil-water storage at each time step $j$ is calculated as

$$
H_{j}=\sum_{i=1}^{n}\left(\theta_{j}^{j}+\theta_{i-1}^{j}\right) \Delta z_{i} / 2
$$

Letting $\Delta I_{j}, \Delta E_{j}$, and $\Delta D_{j}$ denote infiltration, evaporation, and drainage, respectively, during each time step, the water mass balance error is calculated as

$$
\text { error }=\left|\Delta I j-\Delta E_{j}-\Delta D_{j}-\Delta W_{j}\right|
$$

Cumulative surface flux $\left(\Delta Q_{j}\right)$ during the time step $\Delta t_{j}$ is

$$
\Delta Q_{j}=\Delta I_{j}-\Delta E_{j}
$$


The program maintains computational accuracy by adjusting the time step so that the mass balance error Equation (A.6) remains less than a prescribed limit. The continuously changing time step is restricted to specified limits, however. Those limits are provided by the user as minimum and maximum time steps in hours. An option to use a reduced time step during water application is also provided. Model computations proceed with the maximum time step and use a reduced time step as required to maintain the prescribed mass balance error.

The water contents $\theta j$ over the nodes are calculted at each time step from the soil-water characteristic curves associated with each soil type, by using the calculated heads. These curves are represented by polynomials (Appendix 8 ). Internodal fluxes from either hourly or daily periods are reported in the program as integrals over time of

$$
q_{j+1 / 2}^{j-1 / 2}
$$

defined by (A.2). Flux is defined as positive in the downward direction. 
APPENDIX B

POL YNOMIAL DESCRIPTION OF HYORAULIC PROPERTIES 


\section{APPENDIX B}

\section{POLYNOMIAL OESCRIPTION OF HYDRAULIC PROPERTIES}

Because the hydraulic properties of soils exhibit extreme ranges of variation, a special representation in terms of polynomial functions was adopted. For each soil type the suction head domain is divideo by a value $h_{l}$ into wet and dry domains. Within each subdomain the hydraulic properties are expressed as follows:

Soil-water characteristic:

$$
\begin{aligned}
& \theta-\theta_{0}=\sum_{k=1}^{n} a_{k}\left(h-h_{0}\right)^{k}, h_{0} \leq h \leq h_{1} \\
& \theta-\theta_{f}=\sum_{k=1}^{m} b_{k}\left(2 n h-2 n h_{f}\right)^{k}, h_{1} \leq h \leq h_{f}
\end{aligned}
$$

Soil-water capacity:

$$
\begin{aligned}
& c(h)=-\sum_{k=1}^{n} k a_{k}\left(h-h_{0}\right)^{k-1}, h_{0} \leq h \leq h_{1} \\
& c(h)=-\sum_{k=1}^{m} k b_{k}\left(\ell n h-2 n h_{f}\right)^{k-1} / h, h_{1} \leq h \leq h_{f}
\end{aligned}
$$

Conductivity:

$$
\begin{aligned}
& \ln k(h)=\ln k_{0}+\sum_{k=1}^{r} a_{k}\left(h-h_{0}\right)^{k}, h_{0} \leq h \leq h_{1} \\
& \ln k(h)=\ln k_{f}+\sum_{k=1}^{5} b_{k}\left(\ln h-\ln h_{f}\right)^{k}, h_{1} \leq h \leq n_{f}
\end{aligned}
$$


where " $o$ " and " $f$ " denote minimum and maximum values.

Equations (B.1) through (B.3) are defined for positive suction head (h). Coefficients in the equations are evaluated for a specific soil by least squares fitting with the constraint of continuity at the vaiue $h_{1}$. Moreover, the fit of Equation (B.1) for the soil-water characteristic is constrained so that capacity is positive and $\theta$ is monotonic. Such polynomial expressions used in the computer program avoid storage of measurements and provide for simplified extrapolation. These expressions have the advantage of fitting nearly any data set; however, when they are compared to analytical functions describing physical models of hydraulic properties, extrapolation beyond the measurement range generally is not possible. 


\section{APPENOIX C}

CAPILLARY CONDUCTIVITY MODELS 
APPENDIX C

CAPILLARY CONDUCTIVITY MODELS

A program called HYDRAK was developed to calculate the hydraulic conductivity based on the soil-water characteristic curve, according to Mualem's (1976) discussion. The analytic methods of Burdine, Millington-Quirk, and Mualem were included in the program as options.

The measured soil-water characteristic is described by a paired set of water contents and suction heads:

$$
\begin{aligned}
& \theta_{r} \leq \theta_{0} \leq \theta_{1} \leq \theta_{2} \leq \cdots \theta_{n} \leq \theta_{s} \\
& \infty \geq h_{0} \geq h_{1} \geq h_{2} \geq \cdots h_{n} \geq 0
\end{aligned}
$$

where $\theta_{0}$ is the minimum measured water content, and $\theta_{r}$ and $\theta_{S}$ are the residual and saturated water contents, respectively. In the program, the soil-water characteristic is represented between measurements by a line segment or exponential function. The characteristic is divided at a selected point into two domains such that measurements are lineariy connected above the point and exponentialiy connected below.

Letting $\theta^{\prime}$ and $\theta^{\prime \prime}$ denote the limits of any interval, the soil-water characteristic is described as follows:

$$
\begin{aligned}
\text { linear: } h & =B\left(\theta-\theta^{\prime}\right)+h^{\prime}, \theta^{\prime} \leq \theta \leq \theta^{\prime \prime} \\
B & =\left(h^{\prime \prime}-h^{\prime}\right) /\left(\theta^{\prime \prime}-\theta^{\prime}\right) \\
\text { exponential: } \quad \text { ln } h & =B\left(\theta-\theta^{\prime}\right)+\ln h^{\prime} \\
B & =\left(\ln h^{\prime \prime}-\ln h^{\prime}\right) /\left(\theta^{\prime \prime}-\theta^{\prime}\right)
\end{aligned}
$$

Usually the residual water content is not known, but Mualem developed a method to estimate it based on fitting the following equation to the end of a characteristic: 


$$
\frac{\theta-\theta_{r}}{\theta_{0}-\theta_{r}}=\left(\frac{h_{0}}{h}\right)^{\lambda}
$$

$\theta_{r}$ and $\lambda$ are estimated by the least squares fit of (C.I) as discussed by Mualem (1976). The description of the extension, however, is allowed to be a user's option by providing estimated points between $\theta_{0}$ and $\theta_{r}$.

The models are reviewed below. Effective saturation is defined by

$$
S=\left(\theta-\theta_{r}\right) /\left(\theta_{S}-\theta_{r}\right)
$$

$K_{r}$ denotes relative hydraulic conductivity in each model.

- Mualem Modei:

$$
K_{r}(\theta)=s^{1 / 2}\left(\int_{\theta_{r}}^{\theta} d \theta / h / \int_{\theta_{r}}^{\theta} d \theta / h\right)^{2}
$$

Integrals are evaluated as follows:

Linear Case:

$$
\int_{\theta^{\prime}}^{\theta} d \theta / h=\frac{1}{\beta}\left[\ln \left(B\left(\theta-\theta^{\prime}\right)+h^{\prime}\right)-\ln h^{\prime}\right]
$$

for $\theta^{\prime} \leq \theta \leq \theta^{\prime \prime}$, and

$$
\int_{\theta^{\prime}}^{\theta^{\prime \prime}} d \theta / h=\frac{\left(\theta^{\prime \prime}-\theta^{\prime}\right)}{\left(h^{\prime \prime}-h^{\prime}\right)} \ln \left(h^{\prime \prime} / h^{\prime}\right)
$$

Exponential Case:

$$
\int_{\theta^{\prime}}^{\theta} d \theta / h=\frac{1}{\beta h^{\prime}}\left[1-e^{-\beta\left(\theta-\theta^{\prime}\right)}\right]
$$

for $\theta^{\prime} \leq \theta \leq \theta^{\prime \prime}$, and 


$$
\int_{\theta^{\prime}}^{\theta^{\prime \prime}} d \theta / h=\frac{\left(\theta^{\prime \prime}-\theta^{\prime}\right)}{h^{\prime} h^{\prime \prime}}\left(\frac{h^{\prime}-h^{\prime \prime}}{\ln h^{1 / h^{\prime \prime}}}\right)
$$

The case $h^{\prime}=h^{\prime \prime}$ is included as

$$
\int_{\theta^{\prime}}^{\theta^{\prime \prime}} d \theta / h=\left(\theta^{\prime \prime}-\theta^{\prime}\right) / h^{\prime}
$$

Integrals in (C.2) are the sum over those of (C.3) and (C.4)

$$
\int_{\theta_{r}}^{\theta_{i}} d \theta / h=\sum_{j=1}^{j} \int_{\theta_{j-1}^{\theta}}^{\theta_{j}} d \theta / h
$$

letting now $\theta_{r}=\theta_{0}$ and $\theta_{s}=\theta_{n}$. Values of relative conductivity are calculated in the program at $\theta_{i}(i-1, \ldots, n)$ according to $(C .5)$, and values at intermediate points $\theta$ are estimated by interpolation.

- Burdine Model:

$$
K_{r}(\theta)=s^{2} \int_{r}^{\theta} d \theta / h^{2} \int_{\theta_{r}}^{\theta} s d \theta / h^{2}
$$

Integrais are evaluated as follows:

Linear Case:

$$
\int_{\theta^{\prime}}^{\theta^{\prime \prime}} d \theta / h^{2}=\left(\theta^{\prime \prime}-\theta^{\prime}\right) / h^{\prime} h^{\prime \prime}
$$

Exponential Case:

$$
\int_{\theta^{\prime}}^{\theta^{\prime \prime}} d \theta / h^{2}=\frac{\left(\theta^{\prime \prime}-\theta^{\prime}\right)}{2 \ln \left(h^{\prime \prime} / h^{\prime}\right)}\left(h^{1-2}-h^{\prime \prime}-2\right)
$$


$k_{r}$ is calculated as for the Mualem model.

- Millington-Quirk method:

Mualem developed an analytic representation of the classical MillingtonQuirk method (1961), which was originally based on discrete pore classes associated with equal water-content intervals. The formula is

$$
K_{r}(\theta)=S^{m} \int_{\theta_{r}}^{\theta}(\theta-\varepsilon) \frac{d \varepsilon}{h^{2}} / \int_{\theta_{r}}^{\theta}\left(\theta_{S}-\varepsilon\right) \frac{d \varepsilon}{h^{2}}
$$

Intergrals for (C.9) are evaluated as follows:

Linear Case:

$$
\int_{\theta^{\prime}}^{\theta^{\prime \prime}}(\theta-\epsilon) \frac{d \varepsilon}{h^{2}}=\left(\frac{\theta\left(\theta^{\prime \prime}-\theta^{\prime}\right)}{h^{\prime} h^{\prime \prime}}\right)+\left(\frac{\theta^{\prime \prime}-\theta^{\prime}}{h^{\prime \prime}-h^{\prime}}\right)\left(\frac{\theta^{\prime \prime}}{h^{\prime \prime}}-\frac{\theta^{\prime}}{h^{\prime}}\right)+\left(\frac{\theta^{\prime \prime}-\theta^{\prime}}{h^{\prime \prime}-h^{\prime}}\right)^{2} \ln h^{\prime} / h^{\prime \prime}
$$

\section{Exponential Case:}

$$
\begin{aligned}
\int_{\theta^{\prime}}^{\theta^{\prime \prime}}(\theta-\varepsilon) \frac{d \varepsilon}{h^{2}} & =\frac{\theta\left(\theta^{\prime \prime}-\theta^{\prime}\right)}{2 \ln h^{1} / h^{\prime \prime}}\left(h^{\prime \prime-2)}-h^{1-2}\right)-\frac{\left(\theta^{\prime \prime}-\theta^{\prime}\right)}{2 \ln h^{\prime} / h^{\pi}}\left(\theta^{\prime \prime} h^{\prime \prime-2}-\theta^{\prime} h^{1-2}\right) \\
& +\left(\frac{\theta^{\prime \prime}-\theta^{\prime}}{2 \ln h^{\prime} / h^{\prime \prime}}\right)^{2}\left(h^{\prime \prime-2}-h^{1-2}\right)
\end{aligned}
$$

The Millington-Quirk model is defined by $m=4 / 3$ and that of Kunze et al. (1969) by $m=1$. The Childs and Collis-George (1950) method is aiso included by letting $m=0$. A special case when $h^{\prime}=h^{\prime \prime}$ is included as:

$$
\int_{\theta^{\prime}}^{\theta^{\prime \prime}}(\theta-\varepsilon) \frac{d \epsilon}{h^{2}}=\frac{\theta}{h^{\prime 2}}\left(\theta^{\prime \prime}-\theta^{\prime}\right)-\frac{\theta^{\prime \prime 2}-\theta^{\prime 2}}{2 h^{2}}
$$

Again, the integrals in (C.9) are calculated as in the Mualem model. 
No. of

Copies

OFFSITE

A. A. Churm

DOE Patent Division

9800 S. Cass Avenue

Argonne, IL 60439

William E. Mott

Environmental and Safety

Engineering Division

U.S. Department of Energy EP-14

Washington, DC 20545

E. Delaney

Remedial Actions Program

Office of Nuclear Waste

Management

U.S. Department of Energy, NE-301

Washington, DC 20545

Donald H. Groelsema, RAPSO

Office of Nuclear Waste

Management

U.S. Department of Energy

NE-301, GTN

Washington, DC 20545

A. Kluk

Remedial Actions Program

Office of Nuclear Waste

Management

U.S. Department of Energy, NE-301

Washington, DC 20545

Robert W. Ramsey, Jr.

Remedial Actions Program

Office of Nuclear Waste

Management

Washington, DC 20545
No. of

Copies

R. H. Campbell

Uranium Mill Tailings Project

office

U.S. Department of Energy

P.0. Box 5400

Albuquerque, NM 87115

M. L. Mat thews

U.S. Department of Energy

P.0. Box 5400

Albuquerque, NM 87115

27 DOE Technical Information Center

Robert Overmyer

Ford, Bacon \& Davis Utah

375 Chipeta Way

P.0. Box 8009

Salt Lake City, UT 84108

G. F. Birchard

Mail Station 1130-SS

U.S. Nuclear Regulatory Commission

Washington, DC 20555

R. A. Scarano

Uranium Recovery Licence

Branch

Mail Station 483-SS

U.S. Nuclear Regulatory Commission

Washington, DC 20555

2 John W. Mckiernan

Sandia National Laboratory-Albuquerque

P.0. Box 5800

Albuquerque, NM 87185

D. Dreesen

Los Alamos National Scientific Laboratury

P.0. Box 1663

Los Alamos, NM 87545 
No. of

Copies

Walt Kisieleski

Argonne National Laboratory

9700 S. Cass Avenue

Argonne, IL 60439

John D. Nelson

Geotechnical Engineering

Colorado State University

Fort Collins, $\mathrm{CO} 80523$

William Nixon

Office of Nuclear Materials, Safety, and Safeguards

Mail Station 396-SS

U.S. Nuclear Regulatory

Cormission

Washington, OC 20555

J. G. Theme lis

Engineering and Safety Division

DOE Grand Junction Office

2597 B-3/4 Road (South Redlands)

P.0. Box 2567

Grand Junction, CO 81501

E. L. Keller

Technical Services Division

U.S. Department of Energy

Dak Ridge Operations office

P.0. Box E

Oak Ridge, Tennessee 37830

Thomas M. Gerusky

Bureau of Radiation Protection

P.0. Box 2063

Harrisburg, PA 17120

Albert J. Hazle

Radiation and Hazardous Wastes Division

Colorado Department of Health

4210 East 11th Avenue

Denver, CO 80220
No. of

Copies

\author{
Edgar D. Bajley \\ Radiation Control Branch \\ Texas Department of Health \\ $1100 \mathrm{~W}$. 49th Street \\ Austin, TX 78756 \\ Larry Anderson \\ Bureau of Radiation and \\ Occupational Health \\ P.0. Box 2500 \\ Salt Lake City, UT 84110 \\ Walt Ackerman \\ Department of Environmental \\ Quality \\ Land Quality Division \\ Hathaway Building \\ Cheyenne, WY 82002
}

Lynn Fitzrandolph

Arjzona Atomic Energy Commission

2929 West Indian Schoo? Road

Phoenix, AZ 85017

T. N. Narasimhan

Lawrence Berkeley Laboratory

Berkeley, CA 94720

W. Whicker

Department of Radiology and Radiation Biology

Coloardo State U.

Ft. Collin's, CO 80523

Stanley Lichtman

Criteria and Standards

Division

Office of Radiation Programs

U.S. Environmental Protection Agency

Washington, DC 20460

Ted Wolfe

Radiation Department

P.0. Box 968

Santa Fe, NM 87503 
No. of

Copies
No. of

Copies
T. Tamura

Oak Ridge National Laboratory

P.0. Box X

Oak Ridge, TN 37830

A. Ryon

Oak Ridge National Laboratory

P.0. Box X

Oak Ridge, TN 37830

Vern C. Rogers

Rogers \& Associates Engineering

445 East 200 South, Suite 303

Salt Lake City, UT 84111

\author{
A. A. Metry \\ Weston \\ Weston Way \\ West Chester, PA 19380 \\ Battel le Memorial Institute \\ Office of Nuclear Waste \\ I solation \\ Attn: Beverly Rawles \\ 505 King Bivd. \\ Columbus, DH 43210 \\ George Stukenbroeker \\ NLO, Inc . \\ P.0. Box 39158 \\ Cincinnati, Ohio 45239 \\ Alvin Askew \\ Politech Corporation \\ 2220 Austin National Bank \\ Tower \\ Aust in, TX 78701 \\ R. Neff \\ Mound Facility \\ c/o Dayton Area Office \\ P.0. Box 66 \\ Miamisburg, Ohio 45342
}


No. of

Copies

K. R. Porter

Dames and Moore

1626 Cole Blvd.

Golden, CO 80401

ONSITE

2 Richland Operations office

H. E. Ransom

J. White

33 Pacif ic Northwest Laboratory

J. Buelt

L.L. Cadweli

J. F. Cline

G. W. Gee (10)

J. N. Hartley

J. M. Latkovich

R. W. Nelson

C. S. Simmons (10)

Technical Information (5)

Publishing Coordination BE (2) 